\title{
Corpos livres em anéis com divisão
}

\author{
Érica Zancanella \\ TESE APRESENTADA \\ $\mathrm{AO}$ \\ Instituto de Matemática e Estatística \\ DA \\ Universidade De SÃo Paulo \\ PARA \\ OBTENÇÃO DO TÍTULO \\ $\mathrm{DE}$ \\ Doutor em Ciências \\ Área de Concentração: Matemática \\ Orientador: Prof. Dr. Vitor de Oliveira Ferreira
}

Durante o desenvolvimento deste trabalho a autora recebeu auxílio financeiro da CAPES e do CNPq

São Paulo, outubro de 2007 


\title{
Corpos livres em anéis com divisão
}

\author{
Este exemplar corresponde à redação \\ final da tese devidamente corrigida \\ e defendida por Érica Zancanella \\ e aprovada pela Comissão Julgadora.
}

Banca Examinadora:

- Prof. Dr. Vitor de Oliveira Ferreira (orientador) - IME-USP.

- Prof. Dr. Jairo Zacarias Gonçalves - IME-USP.

- Prof. Dr. Plamen Emilov Kochloukov - UNICAMP.

- Prof. Dr. Miguel Angel Alberto Ferrero - UFRGS.

- Prof. Dr. Eduardo Tengan. 


\section{Agradecimentos}

Agradeço primeiramente a Deus pois, sem Ele...

A Virgem Maria e a todos os demais santos aos quais eu recorri durante o doutorado (e não foram poucos)!

Ao Vitor, meu paciente e atencioso orientador, que me apoiou e incentivou quando eu mais precisei, e pelas broncas que ele não me deu.

À banca examinadora pelas sugestões e correções.

Ao Eduardo Tengan e aos professores Jairo, Arnaldo e Makar-Limanov pela disponibilidade, pelas idéias e sugestões.

Ao Marcelo que, como diz o Vitor, é o nosso "consultor para assuntos topológicos".

Ao Lichtman pela atenção, colaboração e pelas idéias que ajudaram a compor o Capítulo 6 deste trabalho.

A minha família pelo incentivo, apoio, segurança e dedicação, sempre.

Ao meu marido, Marcos, que foi "promovido" a marido durante o doutorado, por todo o seu amor e compreensão, pelo apoio e pelas broncas, desde a graduação.

Aos amigos do IME pelo companheirismo, pelos almoços e por agüentarem as minhas reclamações. 


\section{Resumo}

Sejam $D$ um anel com divisão, $K$ um subanel com divisão de $D$ e $X$ um conjunto. O $D$-anel livre sobre $K$ em $X, D_{K}\langle X\rangle=D \underset{K}{*} K\langle X\rangle$, possui um corpo universal de frações denominado corpo livre e denotado por $D_{K} \nless X \ngtr$. Neste trabalho fazemos uma investigação acerca de condições que, quando satisfeitas por um anel com divisão, sejam suficientes para garantir a existência de um subanel isomorfo a algum corpo livre não-comutativo, e também descrevemos famílias de anéis com divisão que satisfazem as condições encontradas. Os anéis com divisão que provamos conter um corpo livre são, em sua maioria, completamentos de corpos de frações de domínios noetherianos com topologia definida por uma valorização.

Palavras-chave: anel, anel com divisão, corpo livre, valorização. 


\begin{abstract}
Let $D$ be a division ring, $K$ a subfield of $D$ and $X$ a set. The $D$-free $\operatorname{ring}$ over $K$ on $X$, $D_{K}\langle X\rangle=D_{K}^{*} K\langle X\rangle$, has an universal field of fractions called a free field and denoted by $D_{K} \nless X \ngtr$. In this work we look into conditions which, when satisfied by a division ring, are sufficient to guarantee the existence of a subring isomorphic to some non-commutative free field, and we also describe families of division rings which satisfy the conditions that were found. The majority of the division rings that we proved to contain a free field are completions of fields of fractions of Noetherian domains with topology defined by a valuation.
\end{abstract}

Keywords: ring, division ring, free field, valuation. 


\section{Sumário}

Lista de Símbolos $\quad$ ix

$\begin{array}{ll}\text { Introdução } & 1\end{array}$

1 Noções Preliminares $\quad 5$

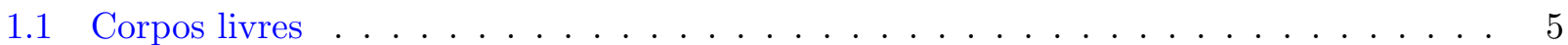

1.2 Anéis clássicos de frações e domínios de Ore . . . . . . . . . . . . . . . . . 13

1.3 Valorizações e completamentos de anéis com divisão . . . . . . . . . . . . . . . 15

1.4 Grupos nilpotentes, finitamente gerados, livres de torção . . . . . . . . . . . . . . 18

1.5 Anéis de grupo e séries de Malcev-Neumann . . . . . . . . . . . . . . . . 21

1.6 Extensões de Ore e séries de Laurent skew . . . . . . . . . . . . . . . . . . . . . . . 22

1.7 Álgebras de Lie e envolventes . . . . . . . . . . . . . . . . . . . . . . . 29

2 Corpos livres em anéis com divisão valorizados $\quad 31$

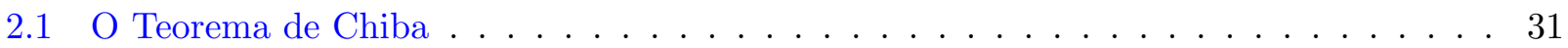

2.2 Anéis com divisão arbitrários . . . . . . . . . . . . . . . . . . . . . 32

2.3 Corpos livres e especializações . . . . . . . . . . . . . . . . . . . . . 35

3 Séries de Malcev-Neumann $\quad 37$

3.1 Uma valorização de $K((G)) \ldots \ldots \ldots \ldots$. . . . . . . . . . . . . 37

vii 
3.2 Corpos livres em $K((G)) \ldots \ldots \ldots \ldots \ldots \ldots \ldots \ldots \ldots$

3.3 Corpos livres em $K((G, \sigma)) \ldots \ldots \ldots \ldots \ldots \ldots \ldots$

4 Séries de Laurent $\quad 49$

4.1 Séries de Laurent skew . . . . . . . . . . . . . . . . . . . . . . . . . . 49

$4.2 \mathrm{O}$ completamento do corpo de Weyl . . . . . . . . . . . . . . . . . . 54

$4.3 \mathrm{O}$ completamento do corpo das matrizes quânticas . . . . . . . . . . . . . 55

5 Uma classe de anéis com valorização t-ádica $\quad 59$

5.1 O completamento de um anel com divisão gerado por um anel com valorização $t$-ádica 59

$5.2 \mathrm{O}$ completamento do corpo de Lie . . . . . . . . . . . . . . . . 60

6 Alguns anéis com divisão que não contêm corpos livres $\quad 65$

$6.1 \mathrm{Um}$ resultado geral . . . . . . . . . . . . . . . . . . . . . . 65

$6.2 \mathrm{O}$ corpo de Weyl . . . . . . . . . . . . . . . . . . . . 72

6.3 O corpo de frações de $K G \ldots \ldots \ldots \ldots \ldots \ldots \ldots \ldots \ldots \ldots \ldots$

$\begin{array}{lll}7 & \text { Considerações Finais } & 77\end{array}$

$\begin{array}{ll}\text { Referências Bibliográficas } & 79\end{array}$

$\begin{array}{ll}\text { Índice Remissivo } & 82\end{array}$ 


\section{Lista de Símbolos}

Vamos fixar algumas notações que serão bastante utilizadas no decorrer da tese. Sejam $R$ um anel, $S \subset R$ um subconjunto, $M$ um $R$-módulo, $K \subset D$ anéis com divisão, $X$ um conjunto e $m, n$ inteiros positivos.

$\begin{array}{ll}\mathbb{N} & \text { O conjunto dos números naturais (incluindo o 0). } \\ \mathbb{Z} & \text { O anel dos números inteiros. } \\ \mathbb{Q} & \text { O corpo dos números racionais. } \\ \mathbb{R} & \text { O corpo dos números reais. } \\ \subset & \text { Usado para inclusão. } \\ \varsubsetneqq & \text { Usado para inclusão estrita. } \\ R^{*} & \text { O conjunto dos elementos não-nulos do anel } R . \\ Z(R) & \text { O centro do anel } R \text {. } \\ \text { Cen } R(S) & \text { O centralizador de } S \text { em } R . \\ \langle S\rangle & \text { O ideal de } R \text { gerado por } S . \\ J(R) & \text { O radical de Jacobson de } R \text {. } \\ \operatorname{Aut}(R) & \text { O grupo dos automorfismos do anel } R . \\ \operatorname{Id}_{M}, 1_{M} & \text { O homomorfismo identidade do } R \text {-módulo } M \text {. } \\ \mathbb{M}_{m \times n}(R),{ }^{n} R^{n} & \text { O anel das matrizes } m \times n \text { sobre } R . \\ I_{n} & \text { A matriz identidade de tamanho } n \times n . \\ M(R) & \text { O anel de matrizes sobre } R \text { de tamanho adequado ao contexto. } \\ I, 1 & \text { A matriz identidade de tamanho adequando ao contexto. } \\ D_{K}\langle X\rangle & \text { O } D \text {-anel livre sobre } X, \text { centralizando } K . \\ D_{K} \nless X \ngtr & \text { O corpo livre. } \\ c a r(D) & \text { A característica do anel com divisão } D .\end{array}$




\section{Introdução}

O interesse na compreensão da estrutura de anéis com divisão tem já uma razoavelmente longa história, mas apenas recentemente resultados mais profundos têm surgido na literatura. Uma das principais conjecturas na área data do final dos anos 70 e foi proposta por Lichtman em [21]: "Seja $D$ um anel com divisão não-comutativo. Então o grupo multiplicativo de $D$ contém um grupo livre não-cíclico". Essa conjectura foi verificada em algumas classes de anéis com divisão; em especial, Gonçalves $[11,12]$ mostrou ser a conjectura verdadeira para anéis com divisão de dimensão finita sobre seus centros. Mais recentemente, Chiba [2] demonstrou que a conjectura é válida para anéis com divisão de centro não-enumerável. Outros trabalhos diretamente relacionados com esse problema são $[10,13,22-24,32,39]$.

Um problema análogo foi proposto por Makar-Limanov em [31]: "Seja $D$ um anel com divisão de dimensão infinita e finitamente gerado sobre seu centro $k$. Então $D$ contém uma subálgebra livre de posto 2 sobre $k$ ". Álgebras livres foram construídas em alguns anéis com divisão conhecidos ([26, 29, 30,33]), mas nenhum método geral foi ainda encontrado.

Nos anos 90, inspirado por trabalhos de Gonçalves e Shirvani [42,43], Makar-Limanov propôs uma conjectura que engloba as duas acima: "Seja $D$ um anel com divisão de dimensão infinita e finitamente gerado sobre seu centro $k$. Então $D$ contém, como subálgebra, a álgebra de grupo do grupo livre de posto 2 sobre $k "$.

Sejam $D$ um anel com divisão, $K$ um subanel com divisão de $D$ e $X$ um conjunto. O $D$-anel livre sobre $K$ em $X, D_{K}\langle X\rangle$, possui um corpo universal de frações denominado corpo livre e denotado por $D_{K} \nless X \ngtr$. Recentemente, Chiba [3] foi capaz de exibir anéis com divisão contendo corpos livres. Se um anel com divisão contém um corpo livre então ele contém a álgebra de grupo de um grupo livre ([20]) e, portanto, contém uma álgebra livre e um grupo livre. O trabalho de Chiba [3] pode ser considerado, assim, como uma contribuição no sentido de iluminar a investigação acerca da existência 
de objetos livres em anéis com divisão. Por outro lado, Lichtman mostrou que o corpo universal de frações da primeira álgebra de Weyl, apesar de conter uma subálgebra livre, não contém um corpo livre.

Neste trabalho, procuramos encontrar condições suficientes para garantir a existência de um subanel isomorfo a um corpo livre em um anel com divisão não-comutativo de dimensão infinita e também descrever famílias de anéis com divisão que satisfaçam tais condições, preferencialmente, buscando incluir os exemplos já encontrados por Chiba. Os anéis com divisão que provamos conter um corpo livre são, em sua maioria, completamentos de corpos de frações de domínios noetherianos com topologia definida por uma valorização.

No Capítulo 1 introduziremos detalhadamente as definições de corpo livre e corpo universal de frações, e apresentaremos várias propriedades relacionadas a esses conceitos. Também nesse capítulo serão apresentados vários outros conceitos e resultados que serão bastante utilizados no decorrer da tese. Embora alguns desses resultados possam ser bastante conhecidos pelo leitor, eles serão introduzidos com o intuito de tornar o trabalho o mais completo possível.

No Capítulo 2 provaremos o Teorema 2.2.4, o qual é uma espécie de generalização do Corolário 1 de [3] para corpos arbitrários, uma vez que tal resultado foi provado em [3] para corpos enumeráveis. Também neste capítulo provaremos um teorema que permite "puxar" corpos livres por meio de uma especialização cujo contradomínio contenha um corpo livre.

No Capítulo 3 aplicaremos o Teorema 2.2.4 para provar a existência de corpos livres em um anel com divisão de séries de Malcev-Neumann de um grupo nilpotente, finitamente gerado, livre de torção, não-abeliano.

No Capítulo 4 aplicaremos o Teorema 2.2.4 para encontrar condições suficientes para garantir a existência de corpos livres em famílias de corpos de séries de Laurent skew. Dentre esses corpos de séries de Laurent estão o completamento do corpo de frações da primeira álgebra de Weyl em característica zero e o completamento do corpo das matrizes quânticas $2 \times 2$ (com topologia definida por uma valorização).

No Capítulo 5 provaremos uma condição suficiente para garantir a existência de um corpo livre em um anel com divisão construído por Lichtman em [25]. Também nesse capítulo provaremos a existência de um corpo livre no completamento de um corpo de frações da envolvente universal de uma álgebra de Lie em característica zero. 
Finalmente, no Capítulo 6, apresentaremos dois exemplos de anéis com divisão que não contêm corpos livres sobre um determinado corpo, embora contenham uma álgebra livre sobre esse mesmo corpo. Tais exemplos nos foram apresentados por Lichtman e aqui serão detalhados.

No decorrer da tese todos os anéis serão associativos, não necessariamente comutativos (salvo menção contrária) e com unidade preservada por homomorfismos, herdada por subanéis e que age como a identidade nos módulos. Um elemento $a$ de um anel $R$ será chamado divisor do zero à esquerd $a$ se existir $b \neq 0$ em $R$ tal que $a b=0$. Divizores do zero à direita são definidos de maneira simétrica. Um elemento que não for um divisor do zero (nem à esquerda nem à direita) será chamado regular. Um anel tal que todos os seus elementos não-nulos forem regulares será chamado domínio. Um anel cujos elementos não-nulos forem todos inversíveis será chamado corpo ou anel com divisão. Para um corpo qualquer $D$, o subcorpo de $D$ gerado por 1 será chamado corpo primo. Por fim, um conjunto $X$ será dito enumerável se existir uma correspondência bijetora entre $X$ e $\mathbb{Z}_{+}$, onde $\mathbb{Z}_{+}$é o conjunto dos números inteiros positivos. 


\section{Capítulo 1}

\section{Noções Preliminares}

Iniciaremos nossos estudos introduzindo alguns conceitos que serão úteis nos capítulos que seguem. Dentre esses conceitos destacamos a definição de "corpo livre", que será apresentada já na Seção 1.1. Nossos resultados provarão a existência de corpos livres em determinados anéis com divisão que, em sua maioria, serão obtidos como completamento de corpos de frações de domínios de Ore. Tal completamento está relacionado com a topologia definida por uma métrica induzida por uma valorização. Para isso apresentaremos nas Seções 1.2 e 1.3 as definições de domínios de Ore e valorizações e algumas de suas propriedades. Destre essas propriedades destacamos que toda valorização definida num domínio de Ore pode ser estendida de maneira única a seu corpo de frações. Alguns domínios de Ore que serão considerados são anéis de grupo de um grupo nilpotente, finitamente gerado, livre de torção. Tal grupo é ordenado e por isso o corpo de frações de seu anel de grupo pode ser imerso num corpo de séries de Malcev-Neumann. Tais definições e resultados serão apresentados nas Seções 1.4 e 1.5. Outros domínios de Ore que também serão considerados são algumas extensões de Ore ou anéis de polinômios skew que, em certos casos, podem ser imersos num corpo de séries de Laurent, como veremos na Seção 1.6. Embora não seja necessariamente um domínio de Ore, a envolvente universal associativa de uma álgebra de Lie também possui um corpo de frações, cujo completamento contém corpo livre, como será mostrado. As definições de álgebra de Lie e envolvente universal serão apresentadas na Seção 1.7. Nem todos os resultados enunciados neste capítulo serão citados no decorrer do texto. Sua inclusão tem o objetivo de minimizar a consulta a outros trabalhos.

\subsection{Corpos livres}

Nesta seção apresentaremos dentre outras coisas a definição de corpo livre, conceito fundamental 
neste trabalho. Todos os conceitos e resultados citados nesta seção podem ser encontrados em [5], [6] e $[8]$.

Seja $R$ um anel. Por um $R$-anel entendemos um anel $L$ com um homomorfismo $R \longrightarrow L$. Para $R$ fixado, os $R$-anéis formam uma categoria onde os morfismos são os homomorfismos de anéis $L \longrightarrow L^{\prime}$ tais que o diagrama

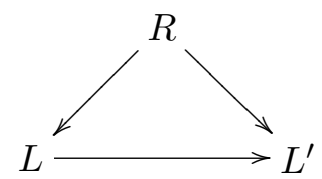

seja comutativo. Um $R$-anel que é um corpo é chamado $R$-corpo. Um $R$-corpo é chamado épico se ele for gerado como corpo pela imagem de $R$. Um $R$-corpo épico $K$ tal que o homomorfismo $R \longrightarrow K$ é injetor, é dito um corpo de frações de $R$.

O único homomorfismo de $R$-anéis possível entre dois $R$-corpos épicos é um isomorfismo. Pois qualquer homomorfismo entre dois corpos deve ser injetor e, neste caso, a imagem será um corpo contendo a imagem de $R$, portanto temos uma sobrejeção. Por esse motivo, é necessário considerar aplicações mais gerais. Define-se um homomorfismo local entre $R$-corpos $K, L$ como um homomorfismo de $R$-anéis $f: K_{0} \longrightarrow L$ de um $R$-subanel $K_{0}$ de $K$ em $L$ tal que todo elemento de $K_{0}$ que não pertence a $\operatorname{Ker}(f)$ tem inverso em $K_{0}$. Segue da definição que $K_{0}$ é um anel local com ideal maximal $\operatorname{Ker}(f)$ e, portanto, $K_{0} / \operatorname{Ker}(f)$ é um corpo isomorfo a $\operatorname{Im}(f) \subset L$. Como $\operatorname{Im}(f)$ é um subcorpo de $L$ contendo a imagem de $R$ em $L$, então se $L$ for um $R$-corpo épico, $\operatorname{Im}(f)=L$. Portanto, todo homomorfismo local em um $R$-corpo épico é sobrejetor. Dois homomorfismos locais de um $R$-corpo $K$ em um $R$-corpo $L$ são considerados equivalentes se eles coincidem sobre um $R$-subanel $K_{0}$ de $K$ e a restrição comum a $K_{0}$ é também um homomorfismo local. Esta relação é de fato uma relação de equivalência. Agora, uma especialização entre dois $R$-corpos $K$ e $L$ é definida como uma classe de equivalência de homomorfismos locais de $K$ em $L$. Freqüentemente escreveremos $f: K \longrightarrow L$ para denotar uma especialização de $K$ em $L$, ficando subentendido que existe um $R$-subanel $K_{0}$ de $K$ e um homomorfismo local $f: K_{0} \longrightarrow L$. Diremos nesse caso que $f: K \longrightarrow L$ é uma especialização com domínio $K_{0}$.

Os $R$-corpos e especializações formam uma categoria denotada por $\mathcal{F}_{R}$. Vejamos como composição de duas especializações é definida. Dadas duas especializações $f: K \longrightarrow L, g: L \longrightarrow M$, sejam $K_{0}, L_{0}$ os domínios de $f$ e $g$, respectivamente, e considere $K_{1}=\left\{x \in K_{0}: f(x) \in L_{0}\right\}, f_{1}=\left.f\right|_{K_{1}}$. Então $K_{1}$ é um $R$-subanel de $K, g f_{1}: K_{1} \longrightarrow M$ é um homomorfismo local e portanto define uma 
especialização de $K$ em $M$.

Seja $\mathcal{E}_{R}$ a subcategoria plena de $\mathcal{F}_{R}$ cujos objetos são os $R$-corpos épicos. Um objeto inicial em $\mathcal{E}_{R}$ é chamado um $R$-corpo universal. Explicitamente, um $R$-corpo universal é um $R$-corpo épico $U$ tal que para todo $R$-corpo épico $K$ existe uma única especialização $U \longrightarrow K$. Claramente um $R$-corpo universal, se existe, é único a menos de isomorfismo. Suponha que $R$ tenha um $R$-corpo universal $U$. Então $R$ tem um corpo de frações se e somente se $U$ é seu corpo de frações; de fato, se $U$ é corpo de frações de $R$ então $R$ tem um corpo de frações. Por outro lado, se $R$ tem um corpo de frações $K$ então existe uma única especialização $f: U \longrightarrow K$. Seja $U_{0}$ o domínio de $f$ e sejam $\lambda_{U}: R \longrightarrow U$ e $\lambda_{K}: R \longrightarrow K$ os homomorfismos canônicos. Então o triângulo

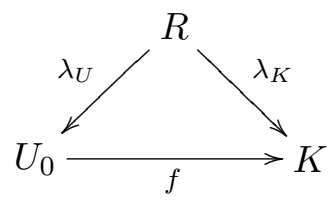

é comutativo. Como $\lambda_{K}$ é injetor, $\lambda_{U}$ é injetor. Assim $U$ é um corpo de frações de $R$. Neste caso, $U$ é dito o corpo universal de frações de $R$.

A existência de $R$-corpos épicos está intrinsicamente relacionada com determinados conjuntos de matrizes sobre $R$, como veremos a seguir.

Definição 1.1.1. Sejam $R$ e $S$ anéis e seja $\Sigma$ um conjunto de matrizes sobre $R$ (de tamanhos arbitrários). Um homomorfismo $f: R \longrightarrow S$ é dito $\Sigma$-inversor se para toda matriz $A$ em $\Sigma, f(A)$ for uma matriz inversivel sobre $S$, onde $f(A)$ denota a matriz sobre $S$ obtida de $A$ por aplicação de $f$ a cada uma de suas entradas.

É fundamental observar que para todo conjunto $\Sigma$ de matrizes sempre existe um homomorfismo $\Sigma$-inversor universal: por este termo entendemos um homomorfismo $\lambda: R \longrightarrow R_{\Sigma}$ que é $\Sigma$-inversor e tal que todo homomorfismo $\Sigma$-inversor $f$ pode ser fatorado de maneira única por $\lambda$, ou seja, dado $f: R \longrightarrow S$ tal que $f(\Sigma)$ consiste de matrizes inversíveis, existe um único homomorfismo $\bar{f}: R_{\Sigma} \longrightarrow S$ tal que o diagrama

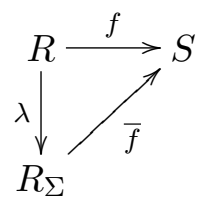


comute. O anel $R_{\Sigma}$ é determinado a menos de isormorfismo por essas condições e é dito o anel $\Sigma$ inversor universal ou também uma localização universal de $R$. Tal anel sempre existe (para quaisquer escolhas de $R$ e $\Sigma$ ) e duas construções distintas podem ser encontradas em [6, pg. 390] e [8, pg. 162]. Temos então o seguinte resultado:

Teorema 1.1.2 ([6], Teorema 7.2.1). Seja $R$ um anel arbitrário e $\Sigma$ um conjunto qualquer de matrizes sobre $R$. Então existe uma localização universal $R_{\Sigma}$, única a menos de isomorfismo, com um homomorfismo $\Sigma$-inversor universal

$$
\lambda: R \longrightarrow R_{\Sigma}
$$

Além disso, $\lambda$ é injetora se e somente se $R$ pode ser imerso num anel sobre o qual todas as matrizes de $\Sigma$ são inversiveis.

Definição 1.1.3. Seja $R$ um anel. Um conjunto $\Sigma$ de matrizes sobre $R$ é dito multiplicativo superior se $1 \in \Sigma$ e sempre que $A, B \in \Sigma$, então $\left(\begin{array}{cc}A & C \\ 0 & B\end{array}\right) \in \Sigma$ para toda matriz $C$ de tamanho apropriado. Conjuntos multiplicativos inferiores são definidos analogamente (com $C$ no canto inferior). Se $\Sigma$ é multiplicativo inferior e toda matriz de $\Sigma$ ainda pertence a $\Sigma$ após qualquer permutação de linhas e colunas, então $\Sigma$ é dito multiplicativo. (Claramente um conjunto multiplicativo é também multiplicativo inferior.)

O próximo teorema mostra como qualquer $R$-corpo épico $K$ pode ser descrito em termos do conjunto das matrizes sobre $R$ que são inversíveis sobre $K$.

Teorema 1.1.4 ([6], Teorema 7.2.2). Seja $R$ um anel. Então

(i) se $\Sigma$ é um conjunto de matrizes tal que a localização universal $R_{\Sigma}$ é um anel local, então o corpo de resíduos de $R_{\Sigma}$ é um $R$-corpo épico e

(ii) se $K$ é um $R$-corpo épico e $\Sigma$ é o conjunto de todas as matrizes sobre $R$ cujas imagens são inversíveis sobre $K$, então $\Sigma$ é multiplicativo e $R_{\Sigma}$ é um anel local com corpo de resíduos isomorfo a $K$.

Vejamos agora como toda especialização também pode ser descrita em termos de matrizes sobre $R$. 
Teorema 1.1.5 ([6], Teorema 7.2.4). Sejam $R$ um anel qualquer, $K_{1}, K_{2} R$-corpos épicos quaisquer, $\Sigma_{i}$ o conjunto de todas as matrizes sobre $R$ que são inversíveis sobre $K_{i}$ e $R_{i}$ a localização universal $R_{\Sigma_{i}}, i=1,2$. Então são equivalentes:

(a) existe uma especialização $K_{1} \longrightarrow K_{2}$,

(b) $\Sigma_{1} \supset \Sigma_{2}$,

(c) existe um homomorfismo de $R$ anéis $R_{2} \longrightarrow R_{1}$.

Se existe uma especialização de $K_{1}$ em $K_{2}$ e uma de $K_{2}$ em $K_{1}$, então $K_{1} \cong K_{2}$.

Uma especialização de $R$-corpos é dita própria se ela não for um isomorfismo. Segue do teorema anterior que

Corolário 1.1.6 ([6], Corolário 7.2.5). Se um R-corpo épico $K$ for uma localização universal, então $K$ não pode ser obtido por uma especialização própria de qualquer outro $R$-corpo.

Em particular, se $R$ tem um $R$-corpo universal $U$, então $U$ é o único $R$-corpo épico que pode ser uma localização universal.

Dentre todas as matrizes sobre um anel $R$ as mais importantes neste trabalho são as matrizes plenas:

Definição 1.1.7. Sejam $R$ um anel e $A \in{ }^{m} R^{n}$, onde ${ }^{m} R^{n}$ denota o conjunto das matrizes $m \times n$ sobre R. A matriz $A$ é dita plena à esquerda se para quaisquer $P \in{ }^{m} R^{r}, Q \in{ }^{r} R^{n}, A=P Q$ implicar $r \geq m$. Matrizes plenas à direita são definidas de modo similar e uma matriz plena à esquerda e à direita é chamada de matriz plena.

Segue da definição acima que uma matriz é plena se e somente se ela for quadrada, digamos $n \times n$, e não puder ser escrita como produto de uma matriz $n \times r$ por uma matriz $r \times n$, com $r<n$.

Definição 1.1.8. Seja $R$ um anel e $A \in{ }^{m} R^{n}$. O posto interno de $A$, denotado por $\rho(A)$, é o menor inteiro $r$ tal que $A=B C$, com $B \in{ }^{m} R^{r}, C \in{ }^{r} R^{n}$.

Observe que se $A \in{ }^{m} R^{n}$ então $A$ é plena à esquerda se e somente se $\rho(A)=m ; A$ é plena à direita se e somente se $\rho(A)=n$. Segue que as matrizes plenas sobre $R$ são exatamente as matrizes 
$n \times n(n \geq 1)$ de posto interno $n$. Quando $R$ é um anel com divisão então as matrizes plenas sobre $R$ constituem exatamente o conjunto das matrizes inversíveis. (Ver [6, pg. 160].)

Definição 1.1.9. Um domínio de Sylvester é um anel não-nulo $R$ tal que para todo n,

$$
P Q=0, \text { com } P \in{ }^{r} R^{n}, Q \in{ }^{n} R^{s}, \text { implicar } \rho(P)+\rho(Q) \leq n \text {. }
$$

Nosso interesse agora é apresentar a classe dos anéis que têm um corpo universal de frações sobre o qual toda matriz plena pode ser invertida.

Um homomorfismo de anéis é chamado de honesto se ele leva matrizes plenas em matrizes plenas. Em particular, um homomorfismo em um corpo $K$ é honesto se ele inverte todas as matrizes plenas. O conjunto de todas as matrizes plenas sobre um anel $R$ será denotado por $\Phi=\Phi(R)$, e um homomorfismo de anéis é dito plenamente inversor se ele for $\Phi$-inversor. Observe que todo homomorfismo plenamente inversor $f: R \longrightarrow S$ em um anel não-nulo $S$ deve ser injetivo, uma vez que todo elemento não-nulo de $R$ é pleno, como uma matriz $1 \times 1$, e então sua imagem por $f$ é um elemento inversível de $S$.

Teorema 1.1.10 (cf. [6], Teorema 7.5.10 e [8], Teorema 4.5.8). Para todo anel $R$ as seguintes condições são equivalentes:

(a) $R_{\Phi}$ é um corpo;

(b) $R$ tem um homomorfismo que preserva posto interno em um corpo;

(c) $R$ é um dominio de Sylvester.

Além disso, $R_{\Phi}$ é o corpo universal de frações de $R$.

Este resultado nos mostra que os domínios de Sylvester formam precisamente a classe de todos os anéis que têm corpo universal de frações sobre o qual toda matriz plena pode ser invertida. (Ver [6, pg. 417].)

Seja $k$ um corpo comutativo. Uma $k$-álgebra livre é descrita facilmente por sua propriedade universal: dado um conjunto $X$ existe uma $k$-álgebra $A=k\langle X\rangle$ e uma função $i: X \longrightarrow A$ tal que toda função $\theta: X \longrightarrow B$ em uma $k$-álgebra $B$ pode ser fatorada unicamente por $i$, ou seja, existe 
um único homomorfismo de $k$-álgebras $\theta^{\prime}: A \longrightarrow B$ tal que o diagrama

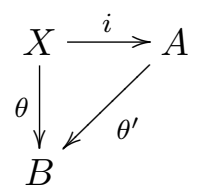

comuta. A menos de isomorfismo, esta álgebra está univocamente determinada pela cardinalidade de $X$. Seus elementos podem ser escritos de maneira única como combinações lineares sobre $k$ de produtos da forma $x_{1} x_{2} \cdots x_{n} \operatorname{com} x_{i} \in X, n \geq 0$, incluindo o produto vazio que é o elemento unidade de $A$. (Ver [6, pg. 59].)

Seja $K$ um corpo não-necessariamente comutativo. Podemos definir o $K$-anel livre $K\langle X\rangle$ sobre um conjunto $X$ pela seguinte propriedade universal: $K\langle X\rangle$ é gerado por $X$ como um $K$-anel e toda função $f: X \longrightarrow R$ em um $K$-anel $R$ tal que $f(x) a=a f(x)$, para todo $a \in K$ e todo $x \in X$, pode ser estendida a um único homomorfismo de $K$-anéis $f^{\prime}: K\langle X\rangle \longrightarrow R$. Este $K$-anel é único a menos de isomorfismo e seus elementos podem ser escritos de maneira única na forma

$$
a_{0}+\sum a_{i_{1} \cdots i_{n}} x_{i_{1}} x_{i_{2}} \cdots x_{i_{n}}
$$

com $a_{0}, a_{i_{1} \cdots i_{n}} \in K, x_{i_{j}} \in X, n \geq 1$. (Ver [5, pg. 111].) Se $K$ for comutativo $K\langle X\rangle$ nada mais é que a $K$-álgebra livre em $X$.

Seja $D$ um corpo com subcorpo $K$ e considere o co-produto $D_{K}^{*} K\langle X\rangle$. (Para qualquer anel $R$ o co-produto na categoria de $R$-anéis de uma família de $R$-anéis $\left(R_{\lambda}\right)$ sempre existe, é denotado por ${ }_{R}^{*} R_{\lambda}$ e é chamado co-produto sobre $R$. Ver [5, pg. 94] e [8, pg. 205].) Esse co-produto nada mais é que o $D$-anel gerado por $X$ com relações $a x=x a$, para $x \in X$ e $a \in K$. (Ver [6, pg.61].) Ele é denotado por $D_{K}\langle X\rangle$ e é chamado $D$-anel livre sobre $K$ em $X$. Segue da definição de co-produto que $D_{K}\langle X\rangle$ tem a seguinte propriedade universal:

Proposição 1.1.11. Seja $R$ um D-anel com homomorfismo canônico $\mu: D \longrightarrow R$. Se $f: X \longrightarrow R$ é uma função tal que $f(x) \mu(a)=\mu(a) f(x)$, para todo $a \in K$ e todo $x \in X$, então existe um único homomorfismo de $D$-anéis $\bar{f}: D_{K}\langle X\rangle \longrightarrow R$ tal que $\bar{f}(x)=f(x)$, para todo $x \in X$.

Observe que quando $D=K$ temos $D_{K}\langle X\rangle=K \underset{K}{*} K\langle X\rangle=K\langle X\rangle$, o $K$-anel livre em $X$.

Teorema 1.1.12. Seja $D$ um anel com divisão com subcorpo $K$, e seja $X$ um conjunto. Então 
o D-anel livre $D_{K}\langle X\rangle$ é um dominio de Sylvester. (Mais precisamente, $D_{K}\langle X\rangle$ é um fir. Ver [6, pg. 114].)

Segue dos dois últimos teoremas que $D_{K}\langle X\rangle$ tem um corpo universal de frações sobre o qual toda matriz plena é inversível. Esse corpo universal de frações é chamado corpo livre e é denotado por $D_{K} \nless X \ngtr$. Quando $D=K$ ele é denotado por $K \nless X \ngtr$.

Vejamos alguns resultados importantes sobre os corpos livres.

Proposição 1.1.13 ([5], Corolário pg. 114). Sejam $K \subset D \subset E$ corpos arbitrários e seja $X$ um conjunto. Então existe uma inclusão natural

$$
D_{K} \nless X \ngtr \longrightarrow E_{K} \nless X \ngtr \text {. }
$$

Proposição 1.1.14. Seja $D$ um corpo com um subcorpo $K$ e seja $D(t)$ o corpo das funções racionais sobre uma indeterminada central t. Então existe uma inclusão natural

$$
D_{K} \nless X \ngtr \longrightarrow D(t)_{K(t)} \nless X \ngtr .
$$

Demonstração: A demonstração é análoga à da Proposição 5.4.4 de [8].

Teorema 1.1.15 ([8], Teorema 5.8.12). Sejam $E \subset D$ anéis com divisão e seja $X$ um conjunto. Então o subcorpo de $D \nless X \ngtr$ gerado por $X$ sobre $E$ é naturalmente isomorfo a $E \nless X \ngtr$.

Teorema 1.1.16 ([8], Teorema 6.4.6). Seja E um corpo com um subcorpo central $C$, seja $D$ um subcorpo de $E$ e seja $k=D \cap C$. Então para qualquer conjunto $X$ existe uma inclusão natural

$$
D_{k} \nless X \ngtr \longrightarrow E_{C} \nless X \ngtr,
$$

se e somente se $D$ e $C$ forem linearmente disjuntos em $E$ sobre $k$ (ou seja, se e somente se o homomorfismo natural $D \otimes_{k} C \longrightarrow$ E for injetor).

Observação 1.1.17. Sejam $R$ e $S$ domínios de Sylvester e denotemos por $\mathcal{U}(R), \mathcal{U}(S)$ seus corpos universais de frações. É importante observar que $R \subset S$ não implica $\mathcal{U}(R) \subset \mathcal{U}(S)$. Na verdade isso acontece se e somente se a inclusão $R \longrightarrow S$ for honesta. (Ver Teorema 1.1.10.) 


\subsection{Anéis clássicos de frações e domínios de Ore}

Na seção anterior introduzimos o conceito de corpo de frações de um anel $R$. Sabemos que todo domínio comutativo possui um corpo de frações, que é único a menos de isomorfismo. No entanto, quando a hipótese de comutatividade não é exigida, este resultado está longe de ser verdadeiro. Existem domínios que não podem ser imersos em nenhum anel com divisão (como por exemplo, a construção de Malcev, que pode ser vista em [19, pg. 290-292]) e portanto não possuem corpos de frações. Por outro lado existem domínios que possuem dois corpos de frações não-isomorfos, como veremos no Capítulo 6, e portanto possuem "mais de um" corpo de frações. No entanto, com algumas hipóteses adicionais, é possível encontrar uma classe de domínios que possuem corpos de frações, únicos a menos de isomorfismo e cujos elementos possuem uma forma normal.

Definição 1.2.1. Um subconjunto $S$ de um anel $R$ é dito ser multiplicativo se $1 \in S, 0 \notin S e$ $a b \in S$, para quaisquer $a, b \in S$.

Definição 1.2.2. Seja $R$ um anel e seja $S \subset R$ um conjunto mulplicativo. Um anel $R^{\prime}$ é dito ser uma localização à direita de $R$ em $S$ ou um anel de frações à direita de $R$ com respeito a $S$ se existir um homomorfismo de anéis $\varphi: R \longrightarrow R^{\prime}$ tal que:

(a) $\varphi$ é $S$-inversor, ou seja, $\varphi(s)$ é inversível, para todo $s \in S$;

(b) todo elemento de $R^{\prime}$ tem a forma $\varphi(a) \varphi(s)^{-1}$, para algum a $\in R$ e algum $s \in S$;

(c) $\operatorname{Ker}(\varphi)=\{r \in R: r s=0$ para algum $s \in S\}$.

Teorema 1.2.3 ([19], Teorema 4.10.6). Seja $R$ um anel e seja $S$ um subconjunto de $R$. Então $R$ possui um anel de frações à direita com respeito a $S$, denotado por $R S^{-1}$, se e somente se as seguintes condições forem satisfeitas:

(1) S é multiplicativo.

(2) Para quaisquer $a \in R, s \in S, a S \cap s R \neq \varnothing$.

(3) Para quaisquer $a \in R, s \in S$, sa $=0$ implicar at $=0$ para algum $t \in S$.

Neste caso, $\varphi: R \longrightarrow R S^{-1}$ é dado por $\varphi(a)=a \cdot 1^{-1}$ e todo elemento de $R S^{-1}$ pode ser escrito na forma $\varphi(a) \varphi(s)^{-1}=a s^{-1}$, para algum $a \in R$ e algum $s \in S$. 
Um subconjunto $S$ de um anel $R$ que satisfaz a condição (2) do teorema acima é dito um conjunto de Ore à direita. Se $S$ satisfaz a condição (3) ele é dito reversível à direita e se ele satisfaz as três condições do teorema ele é dito um conjunto denominador à direita.

Corolário 1.2.4 ([19], pg. 301). Sejam $a_{1} s_{1}^{-1}, a_{2} s_{2}^{-1}, \ldots, a_{n} s_{n}^{-1} \in R S^{-1}$ elementos arbitrários. Então existem $b_{1}, b_{2}, \ldots, b_{n} \in R$ e $s \in S$ tais que $a_{i} s_{i}^{-1}=b_{i} s^{-1}$, para todo $i=1, \ldots, n$. (Expressamos este resultado dizendo que qualquer conjunto finito de elementos de $R S^{-1}$ pode ser colocado num denominador comum.)

Corolário 1.2.5 ([19], Corolário 4.10.11). Seja $S$ um conjunto denominador à direita em R. Então $\varphi: R \longrightarrow R S^{-1}$ é um homomorfismo $S$-inversor universal, ou seja, para todo homomorfismo de anéis $\psi: R \longrightarrow R^{\prime}$ que é $S$-inversor existe um único homomorfismo de anéis $f: R S^{-1} \longrightarrow R^{\prime}$ tal que $\psi=f \varphi$.

De forma análoga também temos as noções de conjunto de Ore à esquerda, conjunto reversível à esquerda e de conjunto denominador à esquerda. Além disso o Teorema 1.2.3 e o Corolário 1.2.5 têm suas respectivas versões à esquerda. Segue do Corolário 1.2 .5 e da sua versão à esquerda o seguinte resultado:

Corolário 1.2.6 ([19], Teorema 4.10.14). Se ambos $R S^{-1}$ e $S^{-1} R$ existem então $R S^{-1} \cong S^{-1} R$.

Seja $S \subset R$ um conjunto multiplicativo. Se $S$ for central em $R$ então, claramente, $S$ é um conjunto denominador à direita e à esquerda. Se $S$ for um conjunto de elementos regulares de $R$ então $S$ é reversível à direita e à esquerda e, neste caso, $\varphi: R \longrightarrow R S^{-1}$ é injetora. Se $S$ consistir de todos os elementos regulares de $R$, dizemos $R$ é um anel de Ore à direita quando $S$ for um conjunto de Ore à direita. Pelo Teorema 1.2.3, isso ocorre se e somente se $R S^{-1}$ existir. Neste caso dizemos que $R S^{-1}$ é o anel clássico de frações à direita de $R$. Os análogos à esquerda dessas noções são definidos similarmente. Se $R$ for um anel de Ore à direita e à esquerda dizemos que $R$ é um anel de Ore. Por exemplo, todo anel comutativo é um anel de Ore, pelas considerações acima.

Seja $R$ um anel e seja $S=R^{*}$, o conjunto dos elementos não-nulos de $R$. Neste caso a condição (2) do Teorema 1.2.3 pode ser escrita da seguinte forma equivalente:

$$
a R \cap b R \neq 0, \text { para quaisquer } a, b \in R^{*} \text {. }
$$

Esta condição é chamada de condição de Ore à direita sobre R. Portanto temos 
Corolário 1.2.7 ([8], Corolário 1.3.3). Seja $R$ um dominio. Então $R$ é um anel de Ore à direita (resp. esquerda) se e somente se $R$ satisfaz a condição de Ore à direita (resp. esquerda). Neste caso, $R$ é dito um domínio de Ore à direita (resp. esquerda), e o anel clássico de frações $R R^{*-1}$ (resp. $R^{*-1} R$ ) é um corpo, chamado corpo clássico de frações à direita (resp. esquerda) de $R$ e é, obviamente, um corpo de frações de $R$.

Proposição 1.2.8 ([8], Proposição 1.3.4). O corpo de frações de um domínio de Ore à direita é unico a menos de isomorfismo.

Proposição 1.2.9 ([8], Proposição 1.3.6). Todo domínio noetheriano à direita é um domínio de Ore à direita.

Definição 1.2.10. Seja $R$ um domínio. Um ideal à direita (resp. esquerda) $I$ de $R$ é dito principal se ele for gerado por um único elemento, ou seja, se $I=a R$ (resp. Ra), para algum a $\in R$. Se todos os ideais à direita (resp. esquerda) de $R$ forem principais $R$ é dito um domínio de ideais principais à direita (resp. esquerda).

Uma vez que todo domínio de ideais principais à direita é um domínio noetheriano à direita $([18,(1.18)$ pg. 20]) temos

Corolário 1.2.11 ([8], Coroloário 1.3.7). Todo domínio de ideais principais à direita é um domínio de Ore à direita e, portanto, tem um (único) corpo de frações.

Por exemplo, se $D$ é um anel com divisão e $z$ é uma indeterminada central então o anel de polinômios $D[z]$ é um domínio de ideais principais à direita e à esquerda e, portanto, é um domínio de Ore com corpo clássico de frações denotado por $D(z)$ e chamado corpo das funções racionais sobre $D$.

\subsection{Valorizações e completamentos de anéis com divisão}

Nesta seção, apresentaremos a definição de valorização e algumas de suas propriedades. Mostraremos também de que forma determinadas valorizações podem induzir métricas em anéis com divisão, tornando-os assim, anéis com divisão topológicos.

Definição 1.3.1. Seja $(G,+)$ um grupo, não necessariamente abeliano. Dizemos que $G$ é ordenado se $G$ possuir uma relação de ordem total $\geq$ tal que

$$
x \geq y, x^{\prime} \geq y^{\prime} \Rightarrow x+x^{\prime} \geq y+y^{\prime}, \text { para quaisquer } x, x^{\prime}, y, y^{\prime} \in G \text {. }
$$


Seja $(G,+)$ um grupo ordenado. Denotemos por 0 o elemento neutro de $G$. Aumentemos $G$ por um símbolo $\infty$, sujeito às regras:

$$
\infty+a=a+\infty=\infty+\infty=\infty, a<\infty \text {, para todo } a \in G .
$$

Então $(G \cup\{\infty\},+)$ é um monóide.

Definição 1.3.2. Uma valorização sobre um anel $R$ com valores em um grupo ordenado $G$ é qualquer aplicação $\nu: R \longrightarrow G \cup\{\infty\}$ satisfazendo as seguintes condições:

(V.1) $\nu(x y)=\nu(x)+\nu(y)$, para todos $x, y \in R$

(V.2) $\nu(x+y) \geq \min \{\nu(x), \nu(y)\}$, para todos $x, y \in R$

(V.3) $\nu(1)=0$ e $\nu(0)=\infty$.

Aplicando a definição obtemos que $\nu(-1)=0$ e portanto $\nu(-x)=\nu(x)$, para todo $x \in R$. Segue facilmente por indução que $\nu\left(\sum_{i=1}^{n} x_{i}\right) \geq \min \left\{\nu\left(x_{i}\right): 1 \leq i \leq n\right\}$. Além disso, se somente um índice $k$ é tal que $\nu\left(x_{k}\right)=\min \left\{\nu\left(x_{i}\right): 1 \leq i \leq n\right\}$ então vale a igualdade acima. $\mathrm{O}$ conjunto $N=\{x \in R: \nu(x)=\infty\}$ é um ideal próprio de $R$, por (V.3). Se $N=0, \nu$ é dita própria; por exemplo, sobre um corpo toda valorização é própria, uma vez que 0 é o único ideal próprio. Daqui em diante, todas as valorizações consideradas serão próprias, salvo menção contrária.

Exemplo 1.3.3. (1) Seja $R$ um anel com um elemento central e regular $t$ tal que $\bigcap t^{n} R=0$ e $R / t R$ é um dominio. Se definimos

$$
\nu_{t}(x)=\sup \left\{n: x \in t^{n} R\right\}
$$

então $\nu_{t}$ é uma valorização de $R$, chamada valorização t-ádica. Por exemplo, se $R$ é um domínio e se $R[z]$ é o anel de polinômios na indeterminada central z sobre $R$, então podemos considerar em $R[z]$ a valorização z-ádica.

(2) Seja $R$ um anel com uma valorização $\nu$ e considere o anel de polinômios $R[z]$. Se definimos

$$
\bar{\nu}\left(\sum a_{i} t^{i}\right)=\min \left\{\nu\left(a_{i}\right)+i\right\},
$$

então $\bar{\nu}$ é uma valorização de $R[z]$ que estende $\nu$. 
Proposição 1.3.4 ([8], Proposição 9.1.1). Seja $R$ um domínio de Ore à direita com corpo de frações $K$. Então toda valorização própria $\nu$ de $R$ tem uma única extensão a uma valorização $\omega$ de $K, a$ saber,

$$
\omega\left(a b^{-1}\right)=\nu(a)-\nu(b), \text { para todos } a, b \in R, b \neq 0 .
$$

Vamos agora considerar uma valorização $\nu$ sobre um corpo $D$. Seja $D^{*}=D-\{0\}$ o grupo multiplicativo de $D$. Segue de (V.1) que $\left.\nu\right|_{D^{*}}$ é um homomorfismo de grupos. Seja $V=\{x \in D$ : $\nu(x) \geq 0\}$. Então $V$ é um subanel de $D$ chamado anel de valorização de $\nu$. Se $x \in V, x \neq 0$, então $0=\nu(1)=\nu\left(x x^{-1}\right)=\nu(x)+\nu\left(x^{-1}\right) \geq \nu\left(x^{-1}\right)$ e portanto $\nu\left(x^{-1}\right) \leq 0$. E, como $\nu: D^{*} \longrightarrow G$ é um homomorfismo de grupos, $\nu\left(x^{-1}\right)=-\nu(x)$. Então $x^{-1} \in V$ se e só se $\nu(x)=0$. Logo o ideal de $V$ definido por $M=\{x \in D: \nu(x)>0\}$ é o conjunto dos elementos não inversíveis de $V$ e portanto $V$ é um anel local. O anel $V / M$ é um anel com divisão chamado corpo de resíduos de $\nu$. A imagem $\nu\left(D^{*}\right)$ sob $\nu$ do grupo multiplicativo $D^{*}$ é um subgrupo de $G$ chamado grupo de valores de $\nu$. Uma valorização $\nu$ sobre $D$ é chamada abeliana se o grupo de valores $\nu\left(D^{*}\right)$ for abeliano e é chamada discreta se o grupo ordenado $\nu\left(D^{*}\right)$ for isomorfo ao grupo aditivo dos inteiros.

Suponhamos que $G$ seja um subgrupo ordenado do grupo aditivo dos números reais. Então podemos definir uma métrica sobre $D$ escolhendo uma constante real $c \in(0,1)$ e definindo

$$
d(x, y)=c^{\nu(x-y)} .
$$

Isso torna $D$ um anel com divisão topológico e, como todo espaço métrico, podemos construir o completamento $\widehat{D}$ de $D$ com relação à topologia definida pela métrica acima. É bem conhecido que $\widehat{D}$ é um anel com divisão com uma valorização $\hat{\nu}: \widehat{D} \longrightarrow G \cup\{\infty\}$, onde $G=\nu\left(D^{*}\right)$, tal que $\widehat{D}$ e $\hat{\nu}$ são extensões de $D$ e $\nu$, respectivamente. (Ver [14], Cap. V, Seção 4 ou [41], Cap. 2.) Chamamos $\widehat{D}$ de completamento de $D$ com relação à topologia definida por $\nu$.

Exemplo 1.3.5. Seja $D$ um anel com divisão e considere o corpo das funções racionais $D(z)$ na indeterminada central z. $D(z)$ nada mais é que o corpo de frações do domínio de Ore $D[z]$. Se $\omega$ é a valorização de $D(z)$ que estende a valorização z-ádica de $D[z]$, então $\omega$ é uma valorização discreta e o completamento de $D(z)$ com relação à topologia definida por $\omega$ é o corpo das séries de Laurent $D((z))$. A métrica em $D((z))$ é a dada pela valorização

$$
\bar{\omega}(f)=\sup \left\{n: f \in z^{n} D[[z]]\right\}, \text { para todo } f \in D((z))
$$


que é uma extensão de $\omega$, onde $D[[z]]$ é o anel das séries de potência em z.

\subsection{Grupos nilpotentes, finitamente gerados, livres de torção}

Dentre os grupos nilpotentes, uma classe se destaca pela existência de uma forma normal para os elementos. Esta classe é a constituída pelos grupos nilpotentes, finitamente gerados, livres de torção. A forma normal dos elementos de um grupo $G$ deste tipo servirá para definirmos uma valorização em anéis de grupo gerados por $G$, como veremos num capítulo posterior. Recordemos primeiramente a definição de grupos nilpotentes.

Definição 1.4.1. Um grupo $G$ é chamado nilpotente se ele contém uma série de subgrupos

$$
\{1\}=G_{0} \subset G_{1} \subset \cdots \subset G_{n}=G
$$

tal que cada subgrupo $G_{i-1}$ é normal em $G$ e cada grupo quociente $G_{i} / G_{i-1}$ está contido no centro de $G / G_{i-1}, 1 \leq i \leq n$.

Uma série de subgrupos de $G$ com essa propriedade é dita uma série central de $G$.

A seguir, apresentaremos alguns resultados sobre grupos nilpotentes que podem ser encontrados, por exemplo, na Seção 1.5 de [37].

Lema 1.4.2. Subgrupos e grupos quocientes de grupos nilpotentes são nilpotentes.

Dados dois elementos $a, b$ de um grupo $G$, o comutador de $a$ e $b$ é o elemento $(a, b):=a^{-1} b^{-1} a b$. O subgrupo derivado $G^{\prime}=(G, G)$ de $G$ é o subgrupo gerado por todos os comutadores $(x, y), x, y \in$ $G$. Obviamente $G$ é abeliano se e somente se $G^{\prime}=\{1\}$. Denotaremos o centro de $G$ por $\mathcal{Z}(G)$. Vejamos as relações entre nilpotência e comutadores. Primeiro, defina duas séries de subgrupos, indutivamente. Por um lado, sejam $\gamma_{1}(G)=G, \gamma_{2}(G)=G^{\prime}$ e $\gamma_{i}(G)=\left(\gamma_{i-1}(G), G\right)$, para todo $i \geq 2$. Por outro lado, sejam $\mathcal{Z}_{0}(G)=\{1\}, \mathcal{Z}_{1}(G)=\mathcal{Z}(G)$ e $\mathcal{Z}_{i}(G)$ como o único subgrupo de $G$ tal que $\mathcal{Z}_{i}(G) / \mathcal{Z}_{i-1}(G)=\mathcal{Z}\left(G / \mathcal{Z}_{i-1}(G)\right)$, para todo $i \geq 1$. O grupo $\mathcal{Z}_{i}(G)$ é chamado o $i$-ésimo centro de $G$.

Definição 1.4.3. As seqüências de subgrupos

$$
\{1\}=\mathcal{Z}_{0}(G) \subset \mathcal{Z}_{1}(G) \subset \cdots \subset \mathcal{Z}_{n}(G) \subset \cdots
$$




$$
G=\gamma_{1}(G) \supset \gamma_{2}(G) \supset \cdots \supset \gamma_{n}(G) \supset \cdots
$$

são chamadas série central superior e série central inferior de $G$, respectivamente.

Claramente, essas séries são séries centrais. Elas são chamadas "superior" e "inferior" pelas seguintes razões:

Lema 1.4.4. Seja

$$
\{1\}=A_{0} \subset A_{1} \subset \cdots \subset A_{n} \subset \cdots
$$

uma série central ascendente arbitrária. Então $A_{n} \subset \mathcal{Z}_{n}(G)$, para todo $n$.

Lema 1.4.5. Seja

$$
G=A_{0} \supset A_{1} \supset \cdots \supset A_{n} \supset \cdots
$$

uma série central descendente arbitrária. Então $\gamma_{n}(G) \subset A_{n-1}$, para todo $n$.

Segue dos lemas acima que um grupo $G$ é nilpotente se e somente se existir $c \geq 0$ tal que $\gamma_{c+1}(G)=\{1\}$ (se e somente se $\mathcal{Z}_{c}(G)=G$ ). O menor inteiro $c$ tal que $\gamma_{c+1}(G)=\{1\}$ é chamado classe de nilpotência de $G$ (e é também o menor inteiro $c$ tal que $\mathcal{Z}_{c}(G)=G$ ).

Proposição 1.4.6. Todo subgrupo de um grupo nilpotente finitamente gerado é finitamente gerado.

Proposição 1.4.7. Seja G um grupo nilpotente livre de torção com série central superior

$$
\{1\}=\mathcal{Z}_{0}(G) \subset \mathcal{Z}_{1}(G) \subset \cdots \subset \mathcal{Z}_{n}(G)=G
$$

Então cada quociente $\mathcal{Z}_{i+1}(G) / \mathcal{Z}_{i}(G)$ é livre de torção. Se, além disso, G é finitamente gerado então $G$ possui pelo menos uma série central

$$
G=G_{1} \supset G_{2} \supset \cdots \supset G_{r+1}=\{1\}
$$

tal que todos os quocientes $G_{j} / G_{j+1}$ são grupos cíclicos infinitos. Uma série central desse tipo será chamada $\mathcal{F}$-série.

Demonstração: Para a demonstração da primeira parte veja o Lema 1.5.26 de [37]. Para a segunda parte, uma vez que $G$ é finitamente gerado, segue que $\mathcal{Z}_{i+1}(G) / \mathcal{Z}_{i}(G)$ é finitamente gerado e livre de torção. Logo $\mathcal{Z}_{i+1}(G) / \mathcal{Z}_{i}(G)$ pode ser escrito como um produto direto de grupos cíclicos infinitos. 
Segue daí que a série central superior pode ser refinada a uma série central com quocientes cíclicos infinitos (ver Corolário 1.5.27 de [37]) .

Seja $G$ um grupo nilpotente, finitamente gerado e livre de torção, e seja

$$
G=F_{1} \supset F_{2} \supset F_{3} \supset \cdots \supset F_{r} \supset F_{r+1}=\{1\}
$$

uma $\mathcal{F}$-série do grupo $G$, ou seja, (1.1) é uma série central de $G$ tal que $F_{i} / F_{i+1},(i=1, \ldots, r)$ é um grupo cíclico infinito. Seja $f_{i}$ um representante em $G$ de um gerador de $F_{i} / F_{i+1}$, ou seja, $F_{i} / F_{i+1}=\left\langle f_{i} F_{i+1}\right\rangle$, o grupo gerado por $f_{i} F_{i+1}$. Então todo elemento de $G$ é escrito de maneira única na forma

$$
g=f_{1}^{\alpha_{1}} f_{2}^{\alpha_{2}} \cdots f_{r}^{\alpha_{r}}
$$

com $\alpha_{1}, \alpha_{2}, \ldots, \alpha_{r} \in \mathbb{Z}$, chamada forma normal de $g$. De fato, seja $g \in G$. Se $g \in F_{r+1}$ então $g=1$. Se $g \in F_{r} \backslash F_{r+1}$ então $g \neq 1$. Logo existe um único $\alpha \in \mathbb{Z}, \alpha \neq 0$, tal que $g=f_{r}^{\alpha}$. Se $g \in F_{r-1} \backslash F_{r}$ então $g F_{r} \neq 1 \cdot F_{r} \in\left\langle f_{r-1} F_{r}\right\rangle$. Logo existe um único $\beta \in \mathbb{Z}, \beta \neq 0$, tal que $g F_{r}=f_{r-1}^{\beta} F_{r}$ o que implica $f_{r-1}^{-\beta} g \in F_{r}$. Então existe $g_{r} \in F_{r}$ tal que $f_{r-1}^{-\beta} g=g_{r}$ e assim $g=f_{r-1}^{\beta} g_{r}$. Mas, como $g_{r} \in F_{r}$, existe um único $\alpha_{r} \in \mathbb{Z}$ tal que $g_{r}=f_{r}^{\alpha_{r}}$. Segue que $g=f_{r-1}^{\beta} f_{r}^{\alpha_{r}}$. Prosseguindo deste modo obtemos que todo elemento $g \in G$ é escrito na forma (1.2). Suponhamos que $g=f_{1}^{\beta_{1}} f_{2}^{\beta_{2}} \cdots f_{r}^{\beta_{r}}$, com $\beta_{i} \in \mathbb{Z}$. Então $f_{1}^{\alpha_{1}-\beta_{1}} f_{2}^{\alpha_{2}} \cdots f_{r}^{\alpha_{r}}=f_{2}^{\beta_{2}} \cdots f_{r}^{\beta_{r}}$ e portanto $f_{1}^{\alpha_{1}-\beta_{1}}=\left(f_{2}^{\beta_{2}} \cdots f_{r}^{\beta_{r}}\right)\left(f_{2}^{\alpha_{2}} \cdots f_{r}^{\alpha_{r}}\right)^{-1} \in F_{2}$. Logo $f_{1}^{\alpha_{1}-\beta_{1}} F_{2}=1 \cdot F_{2}$ o que implica $\alpha_{1}-\beta_{1}=0$, uma vez que $F_{1} / F_{2}$ é livre de torção. Logo $\alpha_{1}=\beta_{1}$ e $\operatorname{assim} f_{2}^{\alpha_{2}} \cdots f_{r}^{\alpha_{r}}=f_{2}^{\beta_{2}} \cdots f_{r}^{\beta_{r}}$. Temos então $f_{2}^{\alpha_{2}-\beta_{2}} f_{3}^{\alpha_{3}} \cdots f_{r}^{\alpha_{r}}=f_{3}^{\beta_{3}} \cdots f_{r}^{\beta_{r}}$ e portanto $f_{2}^{\alpha_{2}-\beta_{2}}=$ $\left(f_{3}^{\beta_{3}} \cdots f_{r}^{\beta_{r}}\right)\left(f_{3}^{\alpha_{3}} \cdots f_{r}^{\alpha_{r}}\right)^{-1} \in F_{3}$. Assim, $f_{2}^{\alpha_{2}-\beta_{2}} F_{3}=1 \cdot F_{3}$ e, portanto, $\alpha_{2}=\beta_{2}$. Prosseguindo de maneira análoga obtemos que $\alpha_{i}=\beta_{i}$, para todo $i=1, \ldots, r$. Portanto todo elemento $g \in G$ é escrito de maneira única na forma (1.2).

Como (1.1) é uma série central então $\left(F_{i}, G\right) \subset F_{i+1}, i=1, \ldots, r$, onde $\left(F_{i}, G\right)$ é o subgrupo gerado por todos os comutadores da forma $(f, g)$ com $f \in F_{i}$ e $g \in G$. Assim, $\left(F_{i}, F_{j}\right) \subset F_{i+1},\left(F_{i}, F_{j}\right)=$ $\left(F_{j}, F_{i}\right) \subset F_{j+1}$ e portanto $\left(F_{i}, F_{j}\right) \subset F_{k}$, onde $k>\max \{i, j\}$. Logo

$$
f_{i}^{\alpha_{i}} f_{j}^{\alpha_{j}}=f_{j}^{\alpha_{j}} f_{i}^{\alpha_{i}} f_{k}^{\gamma_{k}} f_{k+1}^{\gamma_{k+1}} \cdots f_{r}^{\gamma_{r}}
$$

para algum $k>\max \{i, j\}, i, j=1, \ldots, r$, uma vez que $\left(f_{i}^{\alpha_{i}}, f_{j}^{\alpha_{j}}\right) \in\left(F_{i}, F_{j}\right) \subset F_{k}, k>\max \{i, j\}$ e todo elemento de $F_{k}$ é escrito de maneira única na forma $f_{k}^{\gamma_{k}} f_{k+1}^{\gamma_{k+1}} \cdots f_{r}^{\gamma_{r}}$ (pois $F_{k}$ é nilpotente, finitamente gerado, livre de torção e $F_{k} \supset F_{k+1} \supset \cdots \supset F_{r} \supset F_{r+1}=\{1\}$ é uma $\mathcal{F}$-série do grupo 
$\left.F_{k}\right)$. Em particular, $f_{i}^{\alpha_{i}} f_{r}^{\alpha_{r}}=f_{r}^{\alpha_{r}} f_{i}^{\alpha_{i}}$ para todo $i$ e, portanto, $F_{r}=\left\langle f_{r}\right\rangle \subset \mathcal{Z}(G)$, o centro de $G$.

Observe que $G$ pode ser ordenado da seguinte maneira: se $g=f_{1}^{\alpha_{1}} f_{2}^{\alpha_{2}} \cdots f_{r}^{\alpha_{r}}, h=f_{1}^{\beta_{1}} f_{2}^{\beta_{2}} \cdots f_{r}^{\beta_{r}}$ então $g<h$ se existir $s, 1 \leq s \leq r$, tal que $\alpha_{1}=\beta_{1}, \ldots, \alpha_{s-1}=\beta_{s-1}$ e $\alpha_{s}<\beta_{s}$. Esta é a ordem lexicográfica, que é uma relação de ordem total. Segue de (1.3) que se $g<h$ então $g k<h k, k g<k h$ para todo $k \in G$, o que implica que se $g_{1}<h_{1}$ e $g_{2}<h_{2}$ então $g_{1} h_{1}<g_{2} h_{2}$. Logo a relação $\leq$ é compatível com a multiplicação de $G$ e portanto $(G, \leq)$ é um grupo ordenado.

As definições e os resultados que foram apresentados nesta seção podem ser encontrados, por exemplo, em [16] e [37].

\subsection{Anéis de grupo e séries de Malcev-Neumann}

Seja $G$ um grupo e considere o anel de grupo $K G$ sobre um corpo $K$ não necessariamente comutativo. Este é um espaço vetorial sobre $K$ com base $G$ e multiplicação induzida pela multiplicação de $G$. Em certos casos, esse anel pode ser imerso em um anel com divisão. Um desses casos é quando $G$ é um grupo ordenado, por exemplo, quando $G$ é nilpotente, finitamente gerado, livre de torção. Isso seguirá dos resultados mais gerais apresentados a seguir e que podem ser encontrados em [18].

Recordemos que um subconjunto $S$ de um conjunto totalmente ordenado $T$ é dito bem-ordenado se todo subconjunto não-vazio de $T$ possuir um elemento mínimo.

Lema 1.5.1 ([18], Lema 14.17). Sejam S, T subconjuntos bem-ordenados de um conjunto totalmente ordenado $(G,<)$. Então $S \cup T$ é bem-ordenado. Se $(G,<)$ é um grupo ordenado, então

$$
U:=S \cdot T=\{s t: s \in S, t \in T\}
$$

é também bem-ordenado. Além disso, para cada $u \in U$ existe somente um número finito de pares ordenados $(s, t)(s \in S, t \in T)$ tal que $u=s t$.

Seja $G$ um grupo ordenado, com uma ordem $\leq$, seja $R$ um anel e seja $\operatorname{Aut}(R)$ o grupo dos automorfismos de $R$. Fixe um homomorfismo de grupos $\sigma: G \longrightarrow \operatorname{Aut}(R)$ e denote por $\sigma_{g}$ a imagem de $g \in G$ por $\sigma$. Define-se $R((G, \sigma))$ como sendo o conjunto de todas as séries formais (mas não necessariamente finitas)

$$
a=\sum_{g \in G} a_{g} g, a_{g} \in R
$$


com $\operatorname{supp}(a):=\left\{g \in G: a_{g} \neq 0\right\}$ bem-ordenado. O conjunto supp(a) é chamado suporte de $a$. Em $R((G, \sigma))$ nós somamos e multiplicamos os elementos de acordo com as regras:

$$
\begin{gathered}
\sum_{g \in G} a_{g} g+\sum_{g \in G} b_{g} g=\sum_{g \in G}\left(a_{g}+b_{g}\right) g \\
\left(\sum_{g \in G} a_{g} g\right)\left(\sum_{h \in G} b_{h} h\right)=\sum_{l \in G}\left(\sum a_{g} \sigma_{g}\left(b_{h}\right)\right) l,
\end{gathered}
$$

onde a última soma é tomada sobre todos os pares $(g, h)$ tal que $g h=l$. Uma vez que podemos restringir $g$ e $h$ respectivamente a $\operatorname{supp}(a), \operatorname{supp}(b)$, e esses suportes são subconjuntos bem-ordenados de $G$, a última soma acima é finita pelo Lema 1.5.1. Além disso, uma vez que

$$
\begin{gathered}
\operatorname{supp}(a+b) \subset \operatorname{supp}(a) \cup \operatorname{supp}(b), \\
\operatorname{supp}(a b) \subset \operatorname{supp}(a) \cdot \operatorname{supp}(b),
\end{gathered}
$$

então $\operatorname{supp}(a+b)$ e $\operatorname{supp}(a b)$ são ambos bem-ordenados, pelo Lema 1.5.1. Portanto a adição e a multiplicação estão bem-definidas em $R((G, \sigma))$. É possível checar que, com essas operações, $R((G, \sigma))$ é um anel. O subanel de $R((G, \sigma))$ consistindo de todas as somas finitas $\sum a_{g} g$ (ou seja, somas de suporte finito), é chamado anel de grupo skew e é denotado por $R(G, \sigma)$. Se $\sigma$ for o homomorfismo identidade usaremos as notações: $R\left(\left(G, \operatorname{Id}_{R}\right)\right)=R((G))$ e $R\left(G, \operatorname{Id}_{R}\right)=R G$. Neste caso, o subanel $R G$ consistindo das somas finitas nada mais é que o anel de grupo usual.

Teorema 1.5.2. Assuma que $R$ é um anel com divisão e sejam $G$ e $\sigma$ como acima. Então $R((G, \sigma))$ é também um anel com divisão, chamado corpo das séries de Malcev-Neumann skew, se $\sigma \neq \operatorname{Id}_{R} e$ corpo das séries de Malcev-Neumann, se $\sigma=\operatorname{Id}_{R}$.

Demonstração: Ver Teorema 14.21 de [18].

O anel $R((G, \sigma))$ depende, claramente, da relação de ordem considerada em $G$. Mais precisamente, para cada relação de ordem definida em $G$ existe um anel $R((G, \sigma))$.

\subsection{Extensões de Ore e séries de Laurent skew}

Nesta seção, apresentaremos a definição de extensão de Ore e algumas propriedades deste anel. 
Em particular, mostraremos que, em certos casos, esse anéis são domínios de Ore e que seu corpo de frações pode ser imerso num corpo maior, cujos elementos são séries de Laurent. Todos os resultados apresentados nesta seção podem ser encontrados em [8] e [9].

Seja $A$ um anel e $\alpha$ um endomorfismo de $A$. Uma função $\delta: A \longrightarrow A$ é chamada $\alpha$-derivação de $A$ se

$$
\delta(a+b)=\delta(a)+\delta(b), \quad \delta(a b)=\delta(a) \alpha(b)+a \delta(b), \text { para todos } a, b \in A
$$

Proposição 1.6.1 ([8], Proposição 2.1.1). Sejam A um anel, $\alpha$ um endomorfismo e $\delta$ uma $\alpha$ derivação de $A$ e seja $x$ uma indeterminada. Seja $R$ o anel gerado por $x$ e $A$, contendo $A$ como subanel e com relações ax $=x \alpha(a)+\delta(a)$, para todo $a \in A$, ou seja, $R \cong A[x] /\langle a x-x \alpha(a)-\delta(a)\rangle$. Então

(i) cada elemento não-nulo $f \in R$ pode ser escrito unicamente como $f=\sum_{i=0}^{n} x^{i} a_{i}$, para algum inteiro não-negativo $n$ e elementos $a_{i} \in A$, com $a_{n} \neq 0$,

(ii) $R$ é um domínio se A for um dominio e a for injetor,

(iii) se A for um corpo então $R$ é um domínio de ideais principais à direita. Além disso, se $\alpha$ for um automorfismo, $R$ é também um domínio de ideais principais à esquerda.

$\mathrm{O}$ anel $R$ da proposição anterior é chamado extensão de Ore ou anel de polinômios skew em $x$ sobre $A$ associado com $\alpha, \delta$, e é denotado por $A[x ; \alpha, \delta]$. O inteiro $n$ do item (i) acima é chamado o grau de $f$, abreviado $\operatorname{deg}(f)$, e o elemento $a_{n}$ é chamado coeficiente dominante de $f$. Se $f$ tem coeficiente dominante igual a $1, f$ é chamado mônico. O elemento zero de $R$ é definido para ter grau $-\infty$ e coeficiente dominante 0 . Quando $\delta=0$ escrevemos $A[x ; \alpha]$ no lugar de $A[x ; \alpha, 0]$. Se além disso $\alpha=1$, obtemos o anel de polinômios na indeterminada central $x$ sobre $A$, denotado por $A[x]$. O anel $A[x ; 1, \delta]$ é também chamado anel de polinômios diferenciáveis ou anel de operadores diferenciais e é denotado por $A[x ; \delta]$.

O anel $A[x ; \alpha, \delta]$ satisfaz a seguinte propriedade universal:

Proposição 1.6.2. Sejam $A$ e $S$ anéis, $\alpha: A \longrightarrow A$ um endomorfismo e $\delta: A \longrightarrow A$ uma $\alpha$ derivação. Se $\psi: A \longrightarrow S$ é um homomorfismo de anéis e $u \in S$ tem a propriedade que

$$
\psi(a) u=u \psi(\alpha(a))+\psi(\delta(a))
$$


para todo a $\in A$, então existe um único homomorfismo de anéis $\chi: A[x ; \alpha, \delta] \longrightarrow S$ tal que $\chi(x)=u$ e que faz o diagrama

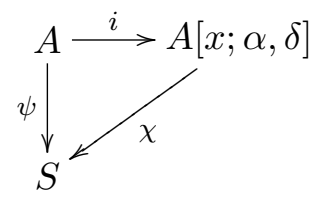

comutar, onde $i: A \longrightarrow A[x ; \alpha, \delta]$ é a inclusão.

Demonstração: Basta definir $\chi: A[x ; \alpha, \delta] \longrightarrow S$ por $\chi\left(\sum x^{i} a_{i}\right)=\sum u^{i} \psi\left(a_{i}\right)$.

É fácil ver que se $A$ for um domínio com um endomorfismo injetor $\alpha$ e uma $\alpha$-derivação $\delta$ então a função deg em $A[x ; \alpha, \delta]$ satisfaz

(i) $\operatorname{deg}(f-g) \leq \max \{\operatorname{deg}(f), \operatorname{deg}(g)\}$,

(ii) $\operatorname{deg}(f g)=\operatorname{deg}(f)+\operatorname{deg}(g)$.

Como todo domínio de ideais principais à direita é um domínio de Ore à direita (pelo Corolário 1.2.11) então, para um corpo $K, K[x ; \alpha, \delta]$ tem um corpo clássico de frações que será denotado por $K(x ; \alpha, \delta)$ e chamado corpo das funções racionais skew. A proposição a seguir mostra exatamente sob quais condições o anel de polinômios skew sobre um corpo é um domínio de ideais principais à esquerda.

Proposição 1.6.3 ([8], Proposição 2.1.6). Seja $K$ um corpo com um endomorfismo $\alpha$ e uma $\alpha$ derivação $\delta$, e seja $R=K[x ; \alpha, \delta]$. Então as seguintes condições são equivalentes:

(a) $\alpha$ é um automorfismo,

(b) $R$ é principal à esquerda,

(c) $R$ é Ore à esquerda.

Sejam $K$ um corpo, $\alpha$ um automorfismo de $K$ e $\delta$ uma $\alpha$-derivação de $K$. Então as seguintes propriedades são satisfeitas pelo anel de polinômios skew $K[x ; \alpha, \delta]$.

Proposição 1.6.4 (Regra de Leibniz, [9], pg.246). Para todo inteiro $n \geq 0$ e para quaisquer elementos a, b, $c \in K$ temos: 
(i) $\delta^{n}(a b)=\sum_{i=0}^{n} \delta^{i}(a) g(i, n-i)(b)$

(ii) $c x^{n}=\sum_{i=0}^{n} x^{i} g(i, n-i)(c)$,

onde $g(i, j): K \longrightarrow K$ é a soma de todas as palavras distintas formadas com $i$ letras $\alpha$ e $j$ letras $\delta$.

Corolário 1.6.5 ([9], pg. 248). Se $\alpha \delta=\delta \alpha$ e $0 \leq i \leq n$,

(i) $g(i, n-i)=\left(\begin{array}{c}n \\ i\end{array}\right) \alpha^{i} \delta^{n-i}$

(ii) $\delta^{n}(a b)=\sum_{i=0}^{n}\left(\begin{array}{c}n \\ i\end{array}\right) \delta^{i}(a) \alpha^{i} \delta^{n-i}(b)$

(iii) $c x^{n}=\sum_{i=0}^{n}\left(\begin{array}{c}n \\ i\end{array}\right) x^{i} \alpha^{i} \delta^{n-i}(c)$.

Como $\alpha$ é um automorfismo é possível escrever os elementos de $K[x ; \alpha, \delta]$ como polinômios em $x$ com coeficientes à esquerda. Mais precisamente, é possível mostrar por indução que, para todo $a \in K$ e todo inteiro $n \geq 0$,

$$
x^{n} a=\sum_{i=0}^{n}(-1)^{n-i} g^{\prime}(i, n-i)(a) x^{i},
$$

onde $g^{\prime}(i, j): K \longrightarrow K$ é a soma de todas as palavras distintas formadas com $i$ letras $\alpha^{-1}$ e $j$ letras $\delta \alpha^{-1}$.

Vamos considerar agora $\delta=0$. Neste caso podemos definir o anel formado pelas séries de potências $\sum_{i=0}^{\infty} x^{i} a_{i}$ com multiplicação induzida por $a x=x \alpha(a)$. Como $\alpha$ é um automorfismo, potências negativas de $\alpha$ estão definidas. Podemos então considerar o anel $K((x ; \alpha))$ das séries de Laurent skew; elas são séries da forma $\sum_{-r}^{\infty} x^{i} a_{i}$, onde $r$ é um inteiro não-negativo, com multiplicação dada pela regra:

$$
a x^{n}=x^{n} \alpha^{n}(a), n \in \mathbb{Z} .
$$

Observe que toda série não-nula pode ser escrita na forma $x^{-r} c\left(1-\sum_{i=1}^{\infty} x^{i} a_{i}\right)$ e esta tem inverso $\left[\sum_{n=0}^{\infty}\left(\sum_{i=1}^{\infty} x^{i} a_{i}\right)^{n}\right] c^{-1} x^{r}$. A soma $\sum_{n=0}^{\infty}\left(\sum_{i=1}^{\infty} x^{i} a_{i}\right)^{n}$ está bem definida, ou seja, é um elemento de $K((x ; \alpha))$, uma vez que as potências de $x$ que aparecem em $\left(\sum_{i=1}^{\infty} x^{i} a_{i}\right)^{n}$ são maiores ou iguais a $n$. Logo $\left[\sum_{n=0}^{\infty}\left(\sum_{i=1}^{\infty} x^{i} a_{i}\right)^{n}\right] c^{-1} x^{r}$ é um elemento de $K((x ; \alpha))$ e, portanto, $K((x ; \alpha))$ é um corpo. Como $K((x ; \alpha)) \supset K[x ; \alpha]$ então $K((x ; \alpha)) \supset K(x ; \alpha)$.

Quando $\delta \neq 0$ não é possível definir um anel formado pelas séries de potências $\sum_{i=0}^{\infty} x^{i} a_{i}$ com multiplicação induzida por $a x=x \alpha(a)+\delta(a)$, uma vez que na multiplicação $b \sum_{i=0}^{\infty} x^{i} a_{i}=\sum_{i=0}^{\infty} b x^{i} a_{i}$, 
$b \in K$, aparecem infinitos termos independentes, por exemplo. Para superar este problema é usual introduzir uma outra variável, $y=x^{-1}$. Segue de $a x=x \alpha(a)+\delta(a)$ que

$$
y a=\alpha(a) y+y \delta(a) y
$$

Aplicando repetidamente esta fórmula obtemos

$$
\begin{aligned}
y a= & \alpha(a) y+\alpha \delta(a) y^{2}+y \delta^{2}(a) y^{2} \\
& \vdots \\
& =\alpha(a) y+\alpha \delta(a) y^{2}+\alpha \delta^{2}(a) y^{3}+\cdots+\alpha \delta^{n-1}(a) y^{n}+y \delta^{n}(a) y^{n} .
\end{aligned}
$$

Se $\delta$ é localmente nilpotente, ou seja, cada elemento de $K$ é anulado por alguma potência de $\delta$, então segue de (1.5) que ya pode ser escrito como um polinômio em y com coeficientes à esquerda. Mas, para qualquer $\delta$, podemos tomar o limite quando $n \rightarrow \infty$ em (1.5) e obter

$$
y a=\alpha(a) y+\alpha \delta(a) y^{2}+\alpha \delta^{2}(a) y^{3}+\alpha \delta^{3}(a) y^{4}+\cdots=\sum_{i=0}^{\infty} \alpha \delta^{i}(a) y^{i+1} .
$$

Denotemos por $K[[y ; \alpha, \delta]]$ o conjunto de todas as séries de potência da forma $\sum_{i=0}^{\infty} a_{i} y^{i}$ e defina em $K[[y ; \alpha, \delta]]$ uma multiplicação induzida por (1.6). Observe que desta vez a multiplicação está bem definida uma vez que na expansão de $y^{n} a$ em potências de $y$ com coeficientes à esquerda, a menor potência de $y$ que aparece é $y^{n}$. Logo $K[[y ; \alpha, \delta]]$ é um anel. Temos então o seguinte teorema:

Teorema 1.6.6 ([8], Teorema 2.3.1). Seja K um anel com divisão com automorfismo $\alpha$ e $\alpha$-derivação $\delta$, e considere o anel

$$
R=K\langle y: y a=\alpha(a) y+y \delta(a) y, \text { para todo } a \in K\rangle .
$$

Então $R \subset K[[y ; \alpha, \delta]]$, que é um dominio. Além disso, o conjunto $\left\{1, y, y^{2}, \ldots\right\}$ é um conjunto de Ore à esquerda em $K[[y ; \alpha, \delta]]$ cuja localização nos dá um corpo $K((y ; \alpha, \delta))$ consistindo de todas as séries de Laurent skew $\sum_{i=r}^{\infty} a_{i} y^{i}, r \in \mathbb{Z}$.

Observação 1.6.7. Como $\alpha$ é um automorfismo, os elementos de $K((y ; \alpha, \delta))$ também podem ser 
escritos na forma $\sum y^{i} a_{i}$, pois

$$
\begin{aligned}
a y & =\left(\alpha\left(\alpha^{-1}(a)\right) y=y \alpha^{-1}(a)-y \delta \alpha^{-1}(a) y=y \alpha^{-1}(a)-y \alpha\left(\alpha^{-1} \delta \alpha^{-1}(a)\right) y\right. \\
& =y \alpha^{-1}(a)-y\left(y \alpha^{-1} \delta \alpha^{-1}(a)-y \delta \alpha^{-1} \delta \alpha^{-1}(a) y\right) \\
& =y \alpha^{-1}(a)-y^{2} \alpha^{-1} \delta \alpha^{-1}(a)+y \delta \alpha^{-1} \delta \alpha^{-1}(a) y \\
& =y \alpha^{-1}(a)-y^{2} \alpha^{-1} \delta \alpha^{-1}(a)+y^{3} \alpha^{-1} \delta \alpha^{-1} \delta \alpha^{-1}(a)-y^{4} \alpha^{-1} \delta \alpha^{-1} \delta \alpha^{-1} \delta \alpha^{-1}(a)+\cdots .
\end{aligned}
$$

Considere o anel de polinômios skew $K[z ; \alpha, \delta]$ na indeterminada $z$. Como $a y^{-1}=a x=x \alpha(a)+$ $\delta(a)$, para todo $a \in K$, então, pela Proposição 1.6.2, existe único homomorfismo de anéis $\psi$ : $K[z ; \alpha, \delta] \longrightarrow K((y ; \alpha, \delta))$ tal que $\psi(z)=y^{-1}=x$ e $\psi(a)=a$, para todo $a \in K$. Se $\psi\left(\sum z^{i} a_{i}\right)=0$ então $\sum x^{i} a_{i}=0$ e, portanto, $a_{i}=0$, para todo $i$. Logo $\sum z^{i} a_{i}=0$ e portanto $\psi$ é injetora. Logo $K[z ; \alpha, \delta] \cong \operatorname{Im}(\psi)$, que é o subanel formado pelos polinômios $\sum_{i=0}^{n} x^{i} a_{i}$. Como $\psi(z)=x$ vamos identificar $z \operatorname{com} x$ e assim temos a inclusão

$$
K[x ; \alpha, \delta] \subset K((y ; \alpha, \delta))
$$

de onde segue que

$$
K(x ; \alpha, \delta) \subset K((y ; \alpha, \delta)) .
$$

Mais ainda, se $f=\sum_{i=-r}^{\infty} a_{i} y^{i} \in K((y ; \alpha, \delta)), r \geq 0$, então

$$
f=\sum_{i=-r}^{-1} a_{i} y^{i}+\sum_{i=0}^{\infty} a_{i} y^{i}=\sum_{i=-r}^{-1} a_{i} x^{-i}+\sum_{i=0}^{\infty} a_{i} y^{i}=\sum_{j=1}^{r} a_{-j} x^{j}+\sum_{i=0}^{\infty} a_{i} y^{i} \in K[x ; \alpha, \delta]+K[[y ; \alpha, \delta]]
$$

e portanto

$$
K((y ; \alpha, \delta))=K[[y ; \alpha, \delta]]+K[x ; \alpha, \delta] .
$$

Vejamos como escrever os elementos de $K(x ; \alpha, \delta)$ como séries de Laurent de $K((y ; \alpha, \delta))$. Sejam $f=\sum_{i=0}^{m} x^{i} a_{i}, g=\sum_{i=0}^{n} x^{i} b_{i} \in K[x ; \alpha, \delta], a_{m}, b_{n} \neq 0, n \geq 1$. Temos

$$
y^{m} f=x^{-m} f=\sum_{i=0}^{m} x^{-m+i} a_{i}=\sum_{i=0}^{m} x^{-(m-i)} a_{i}=\sum_{i=0}^{m} y^{m-i} a_{i}=\sum_{j=0}^{m} y^{j} a_{m-j},
$$




$$
\begin{aligned}
y^{n} g & =x^{-n} g=\sum_{i=0}^{n} x^{-n+i} b_{i}=\sum_{i=0}^{n} x^{-(n-i)} b_{i}=\sum_{i=0}^{n} y^{n-i} b_{i}=\sum_{j=0}^{n} y^{j} b_{n-j} \\
& =b_{n}+y b_{n-1}+\cdots+y^{n-1} b_{1}+y^{n} b_{0}=\left(1+y b_{n-1} b_{n}^{-1}+\cdots+y^{n-1} b_{1} b_{n}^{-1}+y^{n} b_{0} b_{n}^{-1}\right) b_{n} \\
& =\left[1+y\left(b_{n-1} b_{n}^{-1}+\cdots+y^{n-2} b_{1} b_{n}^{-1}+y^{n-1} b_{0} b_{n}^{-1}\right)\right] b_{n}=(1+y h) b_{n},
\end{aligned}
$$

$h=b_{n-1} b_{n}^{-1}+\cdots+y^{n-2} b_{1} b_{n}^{-1}+y^{n-1} b_{0} b_{n}^{-1} \in R$. Seja $l=b_{n}^{-1}\left(1-y h+(y h)^{2}-(y h)^{3}+\cdots\right) \in K[[y ; \alpha, \delta]]$, que é um elemento com termo independende não-nulo. Então

$$
\begin{gathered}
y^{n} g l=(1+y h) b_{n} b_{n}^{-1}\left(1-y h+(y h)^{2}-(y h)^{3}+\cdots\right) \\
=\left(1-y h+(y h)^{2}-(y h)^{3}+\cdots\right)+\left(y h-(y h)^{2}+(y h)^{3}-\cdots\right)=1, \\
l y^{n} g=b_{n}^{-1}\left(1-y h+(y h)^{2}-(y h)^{3}+\cdots\right)(1+y h) b_{n} \\
=b_{n}^{-1}\left(1+y h-y h-(y h)^{2}+(y h)^{2}+(y h)^{3}-(y h)^{3}+\cdots\right) b_{n}=b_{n}^{-1} \cdot 1 \cdot b_{n}=1
\end{gathered}
$$

e portanto $\left(y^{n} g\right)^{-1}=l \in K[[y ; \alpha, \delta]]$. Logo

$$
\begin{aligned}
f g^{-1} & =\left(y^{-m} y^{m}\right) f g^{-1}\left(y^{-n} y^{n}\right)=y^{-m}\left(y^{m} f\right)\left(y^{n} g\right)^{-1} y^{n} \\
& =y^{-m}\left(\sum_{j=0}^{m} y^{j} a_{m-j}\right) l y^{n} \in K((y ; \alpha, \delta)),
\end{aligned}
$$

onde $\left(\sum_{j=0}^{m} y^{j} a_{m-j}\right) l \in K[[x ; \alpha, \delta]]$ e tem termo independente não-nulo.

Um exemplo clássico de anel de polinômios skew é a (primeira) álgebra de Weyl $A_{1}(K)$, sobre um anel com divisão $K$,

$$
A_{1}(K)=K\langle x, y: x y-y x=1\rangle \text {. }
$$

$A_{1}(K)$ pode ser definida como o anel de polinômios skew $K[x]\left[y ; 1, \frac{d}{d x}\right]=K[x]\left[y ; \frac{d}{d x}\right]$, onde $\frac{d}{d x}$ é a derivada formal com relação a $x$. Uma vez que $K[x]$ é um domínio de Ore à direita então $A_{1}(K)=$ $K[x]\left[y ; \frac{d}{d x}\right]$ é um domínio de Ore à direita com corpo clássico de frações $Q\left(A_{1}(K)\right)=K(x)\left(y ; \frac{d}{d x}\right)$, chamado corpo de Weyl. De fato, isto segue do seguinte resultado mais geral.

Observação 1.6.8. Seja $R$ um dominio de Ore à direita com corpo clássico de frações $Q(R)$. Seja $\alpha$ um endomorfismo injetor de $R$ e seja $\delta$ é uma $\alpha$-derivação. Então $\alpha$ se estende a um único 
endomorfismo de $Q(R)$ e $\delta$ se estende a uma única $\alpha$-derivação de $Q(R)$ (pelo Teorema 0.8.11 de [6] ou pela Proposição 2.1.2 de [8]) e temos as inclusões

$$
R[z ; \alpha, \delta] \subset Q(R)[z ; \alpha, \delta] \subset Q(R)(z ; \alpha, \delta)
$$

(ver [6, pg.54]). Com abuso de notação usamos os mesmos símbolos $\alpha$ e $\delta$ para denotar tais extensões. Se $u=f g^{-1} \in Q(R)(z ; \alpha, \delta), f, g \in Q(R)[z ; \alpha, \delta]$, podemos escrever os coeficientes de $f$ e g num denominador comum $c \in R^{*}$ e portanto $f=f_{1} c^{-1}, g=g_{1} c^{-1}$, para alguns $f_{1}, g_{1} \in R[z ; \alpha, \delta]$. Assim, $u=f g^{-1}=\left(f_{1} c^{-1}\right)\left(g_{1} c^{-1}\right)^{-1}=f_{1} g_{1}^{-1}$ e portanto $Q(R)(z ; \alpha, \delta)$ é um corpo clássico de frações de $R[z ; \alpha, \delta]$. Segue que $R[z ; \alpha, \delta]$ é um domínio de Ore à direita.

Vimos que se $R$ for um domínio de Ore à direita e $\alpha$ for um endomorfismo injetor de $R$ então $R[z ; \alpha, \delta]$ é também um domínio de Ore à direita (para qualquer $\alpha$-derivação $\delta$ ). Um resultado semelhante vale no caso em que $R$ é um anel noetheriano à direita.

Proposição 1.6.9 ([6], Proposição 0.10.3). Seja $R$ um dominio noetheriano à direita (resp. esquerda), $\alpha$ um automorfismo de $R$ e $\delta$ uma $\alpha$-derivação de $R$. Então o anel de polinômios skew $R[z ; \alpha, \delta]$ é um domínio noetheriano à direita (resp. esquerda).

Segue dessa proposição que $A_{1}(K)$ é um domínio noetheriano à direita e à esquerda.

\section{7 Álgebras de Lie e envolventes}

Uma álgebra de Lie $L$ sobre um corpo comutativo $k$ é um $k$-espaço vetorial com uma aplicação bilinear $L \times L \longrightarrow L$, comumente chamada multiplicação de Lie ou simplesmente "multiplicação", denotada por $[x, y]$ e satisfazendo as seguintes identidades:

$$
\begin{aligned}
{[x, x] } & =0 \\
{[[x, y], z]+[[y, z], x]+[[z, x], y] } & =0 \quad \text { (identidade de Jacobi). }
\end{aligned}
$$

Segue de (1.8) que $[x, y]=-[y, x]$, ou seja, a multiplicação de Lie é anticomutativa, mas em geral é não-associativa.

Um exemplo importante de uma álgebra de Lie é a derivada de uma álgebra associativa. Seja $A$ 
um anel que também é uma $k$-álgebra e sobre $A$ defina uma multiplicação pela regra

$$
[x, y]=x y-y x .
$$

O $k$-espaço $A$ com a multiplicação (1.10) é uma álgebra de Lie, denotada por $A^{-}$e chamada álgebra de Lie derivada de A.

Se $L$ é uma álgebra de Lie e $A$ é uma álgebra associativa então uma representação de $L$ em $A$ é um homomorfismo de $L$ em $A^{-}$. Um resultado básico que pode ser encontrado, por exemplo, em [15], é que toda álgebra de Lie tem uma representação fiel (injetora) em uma álgebra associativa adequada:

Teorema 1.7.1 (Poincaré-Birkhoff-Witt). Para toda álgebra de Lie L sobre um corpo $k$ existe uma álgebra associativa $U(L)$ com uma representação $i: L \longrightarrow U(L)^{-}$que é universal para representações de $L$ em álgebras associativas. Esta representação é fiel; mais precisamente, se $\left(u_{\lambda}\right)$ é uma base totalmente ordenada de $L$ então $U(L)$ tem uma base constituída pelos monômios da forma

$$
u_{\lambda_{1}} u_{\lambda_{2}} \cdots u_{\lambda_{r}}, \quad \lambda_{1} \leq \lambda_{2} \leq \cdots \leq \lambda_{r}, r=0,1, \ldots
$$

A álgebra $U(L)$ é chamada envolvente universal associativa de $L$ e a representação $i: L \longrightarrow$ $U(L)^{-}$é universal no seguinte sentido: se $A$ é qualquer $k$-álgebra e $\theta: L \longrightarrow A^{-}$é um homomorfismo então existe um único homomorfismo $\theta^{\prime}: U(L) \longrightarrow A$ tal que $\theta=i \theta^{\prime}$.

Um teorema bastante importante provado por Cohn ([8, Teorema 2.6.6]) é que a envolvente universal associativa de qualquer álgebra de Lie $L$ pode ser imersa num anel com divisão $D(L)$, o qual chamaremos de corpo de Lie. Veremos num capítulo posterior que $D(L)$ está contido num corpo maior e que tal corpo contém um corpo livre. 


\section{Capítulo 2}

\section{Corpos livres em anéis com divisão valorizados}

Neste capítulo provaremos dois resultados que nos fornecem condições suficientes para garantir a existência de um subanel isomorfo a um corpo livre em um anel com divisão. O primeiro deles é uma espécie de generalização do Corolário 1 de [3] (Corolário 2.1.2 abaixo) para corpos arbitrários, uma vez que tal resultado foi provado em [3] para corpos enumeráveis. O segundo nos permite "puxar" corpos livres por meio de uma especialização cujo contradomínio contenha um corpo livre.

\subsection{O Teorema de Chiba}

Como citamos na Introdução, um resultado importante sobre a inclusão de corpos livres em anéis com divisão foi provado por Chiba. O Teorema 1 de [3], enunciado abaixo, garante a existência de corpos livres em alguns anéis com divisão completos:

Teorema 2.1.1 ([3], Teorema 1). Seja D um anel com divisão enumerável com centro enumerável $C$, e seja $K$ um subcorpo de $D$ o qual é seu próprio bicentralizador e cujo centralizador $K^{\prime}$ é tal que o $K$-espaço à esquerda $K c K^{\prime}$ é de dimensão infinita sobre $K$, para todo $c \in D^{*}=D-\{0\}$. Se existe uma valorização discreta $\nu$ em $D$ e um elemento não-nulo $t$ de $K^{\prime}$ tal que $\nu(t)>0$, então o completamento $\widehat{D}$ de $D$ com relação à topologia definida por $\nu$ contém um corpo livre $D_{K} \nless X \ngtr$ sobre um conjunto enumerável $X$.

Um caso interessante ocorre quando $K$ é o centro de $D$.

Corolário 2.1.2 ([3], Corolário 1). Seja D um anel com divisão enumerável com centro enumerável $C$ tal que a dimensão de $D$ sobre $C$ seja infinita. Se existe uma valorização discreta $\nu$ em $D$ então 
o completamento $\widehat{D}$ de $D$ com relação à topologia definida por $\nu$ contém o corpo livre $D_{C} \nless X \ngtr$ sobre um conjunto enumerável $X$.

\subsection{Anéis com divisão arbitrários}

Da demonstração do Teorema 1 do artigo [3], Teorema 2.1.1 acima, é possível extrair um resultado um pouco mais geral, no qual não se exige que o anel com divisão $D$ seja enumerável:

Teorema 2.2.1. Seja $D$ um anel com divisão com centro infinito e seja $K$ um subcorpo de $D$ o qual é seu próprio bicentralizador e cujo centralizador $K^{\prime}$ é tal que o K-espaço à esquerda $K c K^{\prime}$ é de dimensão infinita sobre $K$, para todo $c \in D^{*}$. Seja $X$ um conjunto enumerável e seja $\Sigma$ um conjunto enumerável de matrizes plenas sobre $D_{K}\langle X\rangle$. Se existe uma valorização discreta $\nu$ em $D$ e um elemento não-nulo $t$ de $K^{\prime}$ tal que $\nu(t)>0$, então existe um homomorfismo $\Sigma$-inversor $D_{K}\langle X\rangle \longrightarrow \widehat{D}$.

Em particular, se $D$ for enumerável e $\Sigma=\Phi$, o conjunto de todas as matrizes plenas sobre $D_{K}\langle X\rangle$, segue que $\widehat{D}$ contém o corpo livre $D_{K} \nless X \ngtr$, e este é exatamente o Teorema 2.1.1.

O Teorema 2.1.1 tem um corolário importante:

Corolário 2.2.2. Seja $D$ um anel com divisão enumerável com centro enumerável $C$, e seja $K$ um subcorpo de $D$ o qual é seu próprio bicentralizador e cujo centralizador $K^{\prime}$ é tal que o $K$ - espaço à esquerda $K c K^{\prime}$ é de dimensão infinita sobre $K$, para todo $c \in D^{*}$. Então o corpo das séries de Laurent $D((z))$ contém um corpo livre $D_{K} \nless X \ngtr$, sobre um conjunto enumerável $X$.

Este corolário é provado diretamente em [3], sem fazer uso do Teorema 2.1.1. No entanto, é possível obtê-lo como uma conseqüência do Teorema 2.1.1 da seguinte maneira: primeiramente, mostramos que $K(z)$ é igual ao seu bicentralizador e que o $K(z)$-espaço à esquerda $K(z) f K(z)^{\prime}$ é de dimensão infinita sobre $K(z)$ para todo $f \in D(z)^{*}$, onde $K(z)^{\prime}=C_{D(z)}(K(z))=C_{D}(K)(z)=K^{\prime}(z)$. Seja $\omega$ a valorização de $D(z)$ que estende a valorização $z$-ádica de $D[z]$. Então $\omega$ é uma valorização discreta, com $\omega(z)=1>0$ e, como dissemos no Exemplo 1.3.5, o completamento de $D(z)$ com relação à topologia definida por $\omega$ é o corpo das séries de Laurent $D((z))$. Como $D$ é enumerável então $D(z)$ é enumerável. O centro de $D(z)$ é dado pela seguinte

Proposição 2.2.3 ([8], Proposição 2.1.5). Seja $K$ um anel com divisão com centro $C$. Então o corpo das funções racionais $K(t)$ tem centro $C(t)$. 
Pela proposição acima, o centro de $D(z)$ é $C(z)$, que também é enumerável. Logo, pelo Teorema 2.1.1, segue que $D((z))$ contém um corpo livre $D(z)_{K(z)} \varangle X$, sobre um conjunto enumerável $X$. Pela Proposição 1.1.14, $D((z)) \supset D_{K} \nless X \ngtr$.

O Teorema 2.2.1 tem como conseqüência o Corolário 2.1.2 que pode ser generalizado para corpos arbitrários no seguinte sentido:

Teorema 2.2.4. Seja $D$ um anel com divisão com centro infinito $C$ e tal que a dimensão de $D$ sobre $C$ seja infinita. Se existe uma valorização discreta $\nu$ em $D$ então o completamento $\widehat{D}$ de $D$ com relação à topologia definida por $\nu$ contém um corpo livre $C \nless X \ngtr$, sobre um conjunto enumerável $X$.

Para provar o teorema acima precisamos do seguinte lema:

Lema 2.2.5. Todo corpo comutativo infinito contém um subcorpo enumerável.

Demonstração: Seja $C$ um corpo comutativo infinito. Se $\operatorname{car}(C)=0$ então $C \supset \mathbb{Q}$, o corpo dos números racionais. Suponha que $\operatorname{car}(C)=p>0$. Neste caso, o corpo primo de $C$ é $\mathbb{F}_{p}$, o corpo finito com $p$ elementos. Se $C$ for uma extensão algébrica de $\mathbb{F}_{p}$, como $C$ é infinito, $C$ é enumerável. Se existir $x \in C$ transcendente sobre $\mathbb{F}_{p}$ então o corpo das funções racionais $\mathbb{F}_{p}(x)$ é um subcorpo enumerável de $C$.

Precisamos também do seguinte resultado.

Lema 2.2.6 ([3], Lema 9). Seja D um anel com divisão com subcorpo primo П. Seja $k$ um subcorpo central qualquer de $D$ e seja $X$ um subconjunto de D. Então $X$ gera livremente um subcorpo livre $\Pi \nless X \ngtr$ de $D$ sobre $\Pi$ se e somente se ele gera um subcorpo livre $k \nless X \ngtr$ de $D$ sobre $k$.

Demonstração do Teorema 2.2.4: Seja $F \subset C$ um subcorpo enumerável, que existe pelo Lema 2.2.5, e seja $F\langle X\rangle \longrightarrow D_{C}\langle X\rangle$ a inclusão natural. Essa inclusão é honesta, pelo Teorema 1.1.16, uma vez que $F$ e $C$ são obviamente linearmente disjuntos em $D$ sobre $F$. Seja $\Sigma$ o conjunto de todas as matrizes plenas sobre $F\langle X\rangle$. Pelo que acabamos de mostrar, $\Sigma$ está contido em $\Phi$, o conjunto de todas as matrizes plenas sobre $D_{C}\langle X\rangle$. Como $F$ e $X$ são enumeráveis então $\Sigma$ é enumerável. Pelo Teorema 2.2.1, existe um homomorfismo $\Sigma$-inversor $D_{C}\langle X\rangle \longrightarrow \widehat{D}$ e, portanto, a composta

$$
F\langle X\rangle \longrightarrow D_{C}\langle X\rangle \longrightarrow \widehat{D}
$$


é um homomorfismo $\Sigma$-inversor de $F$-anéis. Como $\Sigma$ é o conjunto de todas as matrizes plenas sobre $F\langle X\rangle$ então esse homomorfismo se estende a um homomorfismo de $F$-anéis $F \nless X \ngtr \longrightarrow \widehat{D}$. Logo $X$ gera livremente um subcorpo livre $F \nless X \ngtr$ de $\widehat{D}$ sobre $F$. Como $F \subset C=Z(D) \subset Z(\widehat{D})$, segue pelo Lema 2.2.6 que $X$ gera livremente um subcorpo livre $\Pi \nless X \ngtr$ de $\widehat{D}$ sobre $\Pi$, o subcorpo primo de $D$. Logo $X$ gera livremente um subcorpo livre $C \nless X \ngtr$ de $\widehat{D}$ sobre $C$, novamente pelo Lema 2.2.6.

Corolário 2.2.7. Seja $D$ um anel com divisão com centro $C$ e tal que a dimensão de $D$ sobre $C$ seja infinita. Então o corpo das séries de Laurent $D((z))$ contém um corpo livre $C \nless X \ngtr$ sobre um conjunto enumerável $X$.

Demonstração: Já sabemos que o corpo das funções racionais $D(z)$ tem uma valorização discreta e que seu completamento com relação à topologia definida por essa valorização é $D((z))$. Além disso, pela Proposição 2.2.3, o centro de $D(z)$ é $C(z)$, que é um corpo infinito. Mostremos que $D(z)$ tem dimensão infinita sobre $C(z)$. Suponhamos que $[D(z): C(z)]=n<\infty$ e sejam $d_{1}, \ldots, d_{n+1} \in D \subset D(z)$. Então existem $f_{1} g_{1}^{-1}, \ldots, f_{n+1} g_{n+1}^{-1} \in C(z)$ não todos nulos tais que $f_{1} g_{1}^{-1} d_{1}+\cdots+f_{n+1} g_{n+1}^{-1} d_{n+1}=0$, onde $f_{i}, g_{i} \in C[z], g_{i} \neq 0, i=1, \ldots, n+1$. Sejam $f_{i}^{\prime}, g \in C[z], g \neq 0$, tais que $f_{i} g_{i}^{-1}=f_{i}^{\prime} g^{-1}$, $i=1, \ldots, n+1$. Como os $f_{i} g_{i}^{-1}$ não são todos nulos então os $f_{i}^{\prime}$ não são todos nulos. Como $g$ é central em $D(z)$ segue que

$$
0=f_{1}^{\prime} g^{-1} d_{1}+\cdots+f_{n+1}^{\prime} g^{-1} d_{n+1}=\left(f_{1}^{\prime} d_{1}+\cdots+f_{n+1}^{\prime} d_{n+1}\right) g^{-1}=0
$$

e portanto $f_{1}^{\prime} d_{1}+\cdots+f_{n+1}^{\prime} d_{n+1}=0$. Escreva $f_{i}^{\prime}=\sum_{j} a_{i j} z^{j}, a_{i j} \in C$. Então

$$
0=\left(\sum_{j} a_{1 j} z^{j}\right) d_{1}+\cdots+\left(\sum_{j} a_{n+1, j} z^{j}\right) d_{n+1}=\sum_{j}\left(\sum_{i=1}^{n+1} a_{i j} d_{i}\right) z^{j}
$$

$\operatorname{Logo} \sum_{i=1}^{n+1} a_{i j} d_{i}=0$, para todo $j$. Seja $l \in\{1, \ldots, n+1\}$ tal que $f_{l}^{\prime}=\sum_{j} a_{l j} z^{j} \neq 0$. Então existe $j_{0} \geq 0$ tal que $a_{l j_{0}} \neq 0$. Portanto temos $\sum_{i=1}^{n+1} a_{i j_{0}} d_{i}=0$ com $a_{l j_{0}} \neq 0$, onde $a_{i j_{0}} \in C$ e $d_{i} \in D$, $i=1, \ldots, n+1$. Logo qualquer conjunto com $n+1$ elementos de $D$ é linearmente dependente sobre $C$, contradizendo a hipótese que $D$ tem dimensão infinita sobre $C$. Portanto $[D(z): C(z)]=\infty$. Pelo Teorema 2.2.4, $D((z))$ contém um corpo livre $C(z) \nless X \ngtr$, sobre um conjunto enumerável $X$. Pela Proposição 1.1.14, $D((z)) \supset C \nless X \ngtr$. 


\subsection{Corpos livres e especializações}

O teorema que provaremos a seguir é uma espécie de mecanismo que nos permite "puxar" corpos livres por meio de uma especialização cujo contradomínio contenha um corpo livre.

Teorema 2.3.1. Seja $D$ um anel com divisão com um subcorpo $K$ e seja $E$ um $D$-anel com divisão tal que E contém um corpo livre $D_{K} \nless X \ngtr$. Suponha que exista uma especialização $\alpha: F \longrightarrow E$, onde $F$ é um D-anel com divisão, tal que

(i) $X \subset \operatorname{Im}(\alpha)$;

(ii) para cada $x \in X$ existe $y_{x} \in \alpha^{-1}(x)$ tal que $y_{x} a=$ ayx, para todo $a \in K$.

Então F contém um corpo livre $D_{K} \nless Y \ngtr$, com $|Y|=|X|$.

Demonstração: Seja $F_{0}$ o domínio de $\alpha$. Para cada $x \in X$ fixe $y_{x} \in \alpha^{-1}(x)$ satisfazendo $y_{x} a=a y_{x}$, para todo $a \in K$, e defina o homomorfismo de $D$-anéis $\varphi: D_{K}\langle X\rangle \longrightarrow F_{0}$ tal que $\varphi(x)=y_{x}$. Esquematizando, temos o seguinte diagrama:

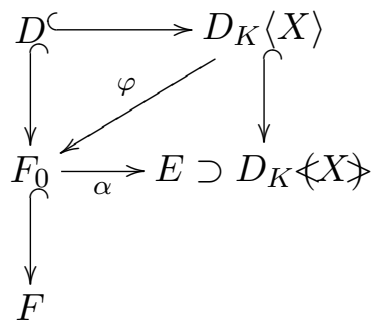

Então $\alpha \varphi(x)=\alpha\left(y_{x}\right)=x$, para todo $x \in X$, e portanto $\alpha \varphi(f)=f$, para todo $f \in D_{K}\langle X\rangle$. Em particular, se $f \in \operatorname{Ker}(\varphi)$ temos $0=\alpha \varphi(f)=f$, donde segue que $\varphi$ é injetora. Logo $D_{K}\langle X\rangle \cong$ $\operatorname{Im}(\varphi) \subset F_{0}$. Escrevendo $Y=\left\{y_{x}: x \in X\right\}$ obtemos que $D_{K}\langle X\rangle \cong D_{K}\langle Y\rangle=\operatorname{Im}(\varphi) \subset F_{0}$. Seja $A\left(y_{x}\right)$ uma matriz plena sobre $D_{K}\langle Y\rangle$. Então $A(x)=\varphi^{-1}\left(A\left(y_{x}\right)\right)$ é plena sobre $D_{K}\langle X\rangle$ e portanto é inversível sobre $D_{K} \Varangle X \gg \subset E$. Agora, observe que $D_{K} \varangle X \gg \subset \operatorname{Im}(\alpha)$; de fato, como $D, X \subset \operatorname{Im}(\alpha)$ então $D_{K}\langle X\rangle \subset \operatorname{Im}(\alpha)$. Além disso, toda matriz plena sobre $D_{K}\langle X\rangle$ é inversível sobre $E$ (equivalentemente, plena sobre $E$ ) e portanto é plena sobre $\operatorname{Im}(\alpha) \subset E$. Como $\operatorname{Im}(\alpha)$ é um corpo, $\operatorname{Im}(\alpha) \cong F_{0} / \operatorname{Ker}(\alpha)$, segue que toda matriz plena sobre $D_{K}\langle X\rangle$ é inversível sobre $\operatorname{Im}(\alpha)$ e, portanto, $D_{K} \Varangle X \gg \subset \operatorname{Im}(\alpha)$. Logo a inversa de $A(x)$ é a imagem de uma matriz $B$ sobre $F_{0}$ : 
$A(x) \alpha(B)=I$. Como $A(x)=\alpha \varphi(A(x))=\alpha\left(A\left(y_{x}\right)\right)$ então $I=\alpha\left(A\left(y_{x}\right)\right) \alpha(B)=\alpha\left(A\left(y_{x}\right) B\right)$. Logo $\alpha\left(A\left(y_{x}\right) B-I\right)=0$ e portanto $A\left(y_{x}\right) B-I=C \in M(\operatorname{Ker}(\alpha))=M\left(J\left(F_{0}\right)\right)=J\left(M\left(F_{0}\right)\right)$, onde $M(R)$ é o anel de matrizes de tamanho apropriado sobre um anel $R$ e $J(R)$ é o radical de Jacobson de $R$. Logo $A\left(y_{x}\right) B=I+C$ é inversível sobre $F_{0}$ e assim $A\left(y_{x}\right) B(I+C)^{-1}=I \Rightarrow A\left(y_{x}\right)$ é inversível à direita sobre $F_{0} \Rightarrow A\left(y_{x}\right)$ é inversível à direita sobre $F$. Como $F$ é um corpo segue que $A\left(y_{x}\right)$ é inversível sobre $F$. Portanto $F$ contém o corpo livre $D_{K} \nless Y \ngtr$. 


\section{Capítulo 3}

\section{Séries de Malcev-Neumann}

Seja $K G$ a álgebra de grupo sobre um corpo comutativo $K$ de um grupo nilpotente, finitamente gerado, livre de torção, não-abeliano $G$. Como vimos na Seção 1.4, $G$ é ordenável e portanto podemos considerar o corpo das séries de Malcev-Neumann $K((G))$ relativo a uma ordem fixada de $G$. Uma vez que $K G \subset K((G))$ então $K G$ é um domínio. Além disso, segue do Corolário 10.2.8 de [34] que $K G$ é um anel noetheriano. Em particular, $K G$ é um domínio de Ore e seu corpo clássico de frações será denotado por $Q(K G)$. Temos então as inclusões $K G \subset Q(K G) \subset K((G))$. De agora em diante, até o final deste trabalho, $G$ será considerado com a relação de ordem definida na Seção 1.4. Provaremos neste capítulo que o corpo das séries de Malcev-Neumann $K((G))$ contém um corpo livre sobre o centro de $Q(K G)$. Isso é uma aplicação do Teorema 2.2.4.

\subsection{Uma valorização de $K((G))$}

Como todo elemento de $G$ é escrito de maneira única na forma (1.2), temos

$$
K G=\left\{\sum_{I} a_{I} f_{1}^{\alpha_{1}} f_{2}^{\alpha_{2}} \cdots f_{r}^{\alpha_{r}}: I=\left(\alpha_{1}, \alpha_{2}, \ldots, \alpha_{r}\right) \in \mathbb{Z}^{r}, a_{I} \in K,\left(a_{I}\right) \text { quase-nula }\right\} .
$$

Definamos sobre $K G$ a função $o_{f_{1}}: K G \longrightarrow \mathbb{Z} \cup\{\infty\}$ dada por: se $f$ é um elemento não-nulo de $K G, f=\sum_{I} a_{I} f_{1}^{\alpha_{1}} f_{2}^{\alpha_{2}} \cdots f_{r}^{\alpha_{r}}$, então

$$
\begin{aligned}
o_{f_{1}}(f) & =\min \left\{\alpha_{1}: f_{1}^{\alpha_{1}} f_{2}^{\alpha_{2}} \cdots f_{r}^{\alpha_{r}} \in \operatorname{supp}(f) \text { para alguns } \alpha_{2}, \ldots, \alpha_{r} \in \mathbb{Z}\right\} \\
& =\min \left\{\alpha_{1}: a_{\left(\alpha_{1}, \alpha_{2}, \ldots, \alpha_{r}\right)} \neq 0 \text { para alguns } \alpha_{2}, \ldots, \alpha_{r} \in \mathbb{Z}\right\}
\end{aligned}
$$


e $o_{f_{1}}(0)=\infty$. Vamos chamar $o_{f_{1}}$ de função ordem com relação a $f_{1}$.

Lema 3.1.1. o $o_{1}$ é uma valorização de $K G$.

Demonstração: Temos $o_{f_{1}}(1)=0$. Sejam $f=\sum_{I} a_{I} f_{1}^{\alpha_{1}} f_{2}^{\alpha_{2}} \cdots f_{r}^{\alpha_{r}}$ e $g=\sum_{I} b_{I} f_{1}^{\alpha_{1}} f_{2}^{\alpha_{2}} \cdots f_{r}^{\alpha_{r}}$ elementos de $K G$ com $o_{f_{1}}(f)=s \in \mathbb{Z}$ e $o_{f_{1}}(g)=t \in \mathbb{Z}$. Temos

$$
f+g=\sum\left(a_{I}+b_{I}\right) f_{1}^{\alpha_{1}} f_{2}^{\alpha_{2}} \cdots f_{r}^{\alpha_{r}} .
$$

Se $f+g=0$ então $o_{f_{1}}(f+g)=\infty>\min \left\{o_{f_{1}}(f), o_{f_{1}}(g)\right\}$. Suponhamos então que $f+g \neq 0$ e suponhamos sem perda de generalidade que $s \leq t$. Seja $\mathcal{I}=\left\{I=\left(\alpha_{1}, \alpha_{2}, \ldots, \alpha_{r}\right) \in \mathbb{Z}^{r}: \alpha_{1}=s\right\}$. Se $a_{I}+b_{I}=0$ para todo $I \in \mathcal{I}$ então $o_{f_{1}}(f+g)>s=\min \{s, t\}$. Se $a_{I}+b_{I} \neq 0$ para algum $I \in \mathcal{I}$ então $o_{f_{1}}(f+g)=s=\min \{s, t\}$. Logo, $o_{f_{1}}(f+g) \geq \min \left\{o_{f_{1}}(f), o_{f_{1}}(g)\right\}$. Se $f=0$ ou $g=0$ então $o_{f_{1}}(f+g)=\min \left\{o_{f_{1}}(f), o_{f_{1}}(g)\right\}$.

Agora, segue de (1.3) que

$$
\begin{aligned}
f g & =\sum_{I, J} a_{I} b_{J} f_{1}^{\alpha_{1}} f_{2}^{\alpha_{2}} \cdots f_{r-1}^{\alpha_{r-1}} f_{1}^{\beta_{1}} f_{2}^{\beta_{2}} \cdots f_{r-1}^{\beta_{r-1}} f_{r}^{\alpha_{r}+\beta_{r}} \\
& =\sum_{I, J} a_{I} b_{J} f_{1}^{\alpha_{1}} f_{2}^{\alpha_{2}} f_{1}^{\beta_{1}} f_{2}^{\beta_{2}} f_{3}^{\alpha_{3}+\beta_{3}} f_{4}^{\gamma_{4}} \cdots f_{r}^{\gamma_{r}} \\
& =\sum_{I, J} a_{I} b_{J} f_{1}^{\alpha_{1}+\beta_{1}} f_{2}^{\alpha_{2}} f_{3}^{\epsilon_{3}} \cdots f_{r}^{\epsilon_{r}} f_{2}^{\beta_{2}} f_{3}^{\alpha_{3}+\beta_{3}} f_{4}^{\gamma_{4}} \cdots f_{r}^{\gamma_{r}} \\
& =\sum_{I, J} a_{I} b_{J} f_{1}^{\alpha_{1}+\beta_{1}} f_{2}^{\alpha_{2}+\beta_{2}} f_{3}^{\alpha_{3}+\beta_{3}+\epsilon_{3}} f_{4}^{\phi_{4}} \cdots f_{r}^{\phi_{r}} .
\end{aligned}
$$

Como $o_{f_{1}}(f)=s$ e $o_{f_{1}}(g)=t$ existem $I_{0}=\left(s, s_{2}, \ldots, s_{r}\right), J_{0}=\left(t, t_{2}, \ldots, t_{r}\right)$ tais que $a_{I_{0}} \neq 0$ e $b_{J_{0}} \neq 0$ e portanto $a_{I_{0}} b_{J_{0}} \neq 0$. Tomemos $I_{0}$ e $J_{0}$ de forma que $f_{1}^{s} f_{2}^{s_{2}} \cdots f_{r}^{s_{r}}=\min \operatorname{supp}(f)$ e $f_{1}^{t} f_{2}^{t_{2}} \cdots f_{r}^{t_{r}}=\min \operatorname{supp}(g)$. Suponhamos que

$$
f_{1}^{\alpha_{1}} f_{2}^{\alpha_{2}} \cdots f_{r}^{\alpha_{r}} f_{1}^{\beta_{1}} f_{2}^{\beta_{2}} \cdots f_{r}^{\beta_{r}}=f_{1}^{s} f_{2}^{s_{2}} \cdots f_{r}^{s_{r}} f_{1}^{t} f_{2}^{t_{2}} \cdots f_{r}^{t_{r}}
$$

ou seja,

$$
f_{1}^{\alpha_{1}+\beta_{1}} f_{2}^{\alpha_{2}+\beta_{2}} f_{3}^{\alpha_{3}+\beta_{3}+\epsilon_{3}} f_{4}^{\phi_{4}} \cdots f_{r}^{\phi_{r}}=f_{1}^{s+t} f_{2}^{s_{2}+t_{2}} f_{3}^{s_{3}+t_{3}+\epsilon_{3}^{\prime}} f_{4}^{\phi_{4}^{\prime}} \cdots f_{r}^{\phi_{r}^{\prime}},
$$

para algum $I=\left(\alpha_{1}, \alpha_{2}, \ldots, \alpha_{r}\right)$ e algum $J=\left(\beta_{1}, \beta_{2}, \ldots, \beta_{r}\right)$, tais que $a_{I}, b_{J} \neq 0$. Então $\alpha_{1}+\beta_{1}=$ $s+t, \alpha_{2}+\beta_{2}=s_{2}+t_{2}, \alpha_{3}+\beta_{3}+\epsilon_{3}=s_{3}+t_{3}+\epsilon_{3}^{\prime}, \phi_{4}=\phi_{4}^{\prime}, \ldots, \phi_{r}=\phi_{r}^{\prime}$. Suponhamos por absurdo que 
$\alpha_{1}, \beta_{1}$ são tais que $\alpha_{1} \neq s, \beta_{1} \neq t$. Então $\alpha_{1}<s$ ou $\beta_{1}<t$. Se $\alpha_{1}<s$ então $a_{I}=0$. Se $\beta_{1}<t$ então $b_{I}=0$. Portanto devemos ter $\alpha_{1}=s$ e $\beta_{1}=t$. Se $\alpha_{2} \neq s_{2}\left(\Rightarrow \beta_{2} \neq t_{2}\right)$ então $\alpha_{2}<s_{2}$ ou $\beta_{2}<t_{2}$. Se $\alpha_{2}<s_{2}$ então $a_{I}=0$. Se $\beta_{2}<t_{2}$ então $b_{I}=0$. Portanto devemos ter $\alpha_{2}=s_{2}$ e $\beta_{2}=t_{2}$. Segue que $\epsilon_{3}=\epsilon_{3}^{\prime}$ e assim $\alpha_{3}+\beta_{3}=s_{3}+t_{3}$. De modo análogo segue que $\alpha_{3}=s_{3}$ e $\beta_{3}=t_{3}$. Prosseguindo dessa forma obtemos que o elemento $f_{1}^{s} f_{2}^{s_{2}} \cdots f_{r}^{s_{r}} f_{1}^{t} f_{2}^{t_{2}} \cdots f_{r}^{t_{r}}=f_{1}^{s+t} f_{2}^{s_{2}+t_{2}} f_{3}^{s_{3}+t_{3}+\epsilon_{3}^{\prime}} f_{4}^{\phi_{4}^{\prime}} \cdots f_{r}^{\phi_{r}^{\prime}}$ aparece uma única vez no suporte de $f g$. Logo

$$
\begin{aligned}
f g= & a_{I_{0}} b_{J_{0}} f_{1}^{s+t} f_{2}^{s_{2}+t_{2}} f_{3}^{\phi_{3}{ }^{\prime}} \cdots f_{r}^{\phi_{r}{ }^{\prime}}+\sum_{\substack{I \neq I_{0} \\
J}} a_{I} b_{J} f_{1}^{\alpha_{1}+\beta_{1}} f_{2}^{\alpha_{2}+\beta_{2}} f_{3}^{\phi_{3}} f_{4}^{\phi_{4}} \cdots f_{r}^{\phi_{r}} \\
& +\sum_{\substack{I \\
J \neq J_{0}}} a_{I} b_{J} f_{1}^{\alpha_{1}+\beta_{1}} f_{2}^{\alpha_{2}+\beta_{2}} f_{3}^{\phi_{3}} f_{4}^{\phi_{4}} \cdots f_{r}^{\phi_{r}}
\end{aligned}
$$

com $\alpha_{1} \geq s, \beta_{1} \geq t$ e portanto $o_{f_{1}}(f g)=s+t=o_{f_{1}}(f)+o_{f_{1}}(g)$. Portanto $o_{f_{1}}$ é uma valorização de $K G$.

Seja $\nu: Q(K G) \longrightarrow \mathbb{Z} \cup\{\infty\}$ a única valorização de $Q(K G)$ tal que $\left.\nu\right|_{K G}=o_{f_{1}}$; mais precisamente, se $f g^{-1} \in Q(K G), f, g \in K G$, então $\nu\left(f g^{-1}\right)=o_{f_{1}}(f)-o_{f_{1}}(g)$, pela Proposição 1.3.4. Fixemos uma constante real $c \in(0,1)$ e definamos sobre $Q(K G)$ a seguinte métrica:

$$
d(u, v)=c^{\nu(u-v)}, u, v \in Q(K G) .
$$

Vamos estudar o completamento de $Q(K G)$ com relação à topologia dada por essa métrica.

Considere o corpo das séries de Malcev-Neuman $K((G))$. Observe que se $f \in K((G))$ é um elemento não-nulo, então $a_{I}=a_{\left(\alpha_{1}, \ldots, \alpha_{r}\right)} \neq 0$ somente para um número finito de valores negativos de $\alpha_{1}$ pois, caso contrário, supp $(f)$ não teria um elemento mínimo. Podemos então escrever

$$
f=\sum_{\substack{I=\left(\alpha_{1}, \ldots, \alpha_{r}\right) \\ \alpha_{1} \geq \alpha_{0}}} a_{I} f_{1}^{\alpha_{1}} \cdots f_{r}^{\alpha_{r}},
$$

para algum $\alpha_{0} \in \mathbb{Z}$.

Definamos sobre $K((G))$ a função $\hat{\nu}: K((G)) \longrightarrow \mathbb{Z} \cup\{\infty\}$ dada por: se $f=\sum a_{I} f_{1}^{\alpha_{1}} f_{2}^{\alpha_{2}} \cdots f_{r}^{\alpha_{r}} \in$ 
$K((G))$ é um elemento não-nulo então

$$
\begin{aligned}
\hat{\nu}(f) & =\min \left\{\alpha_{1}: f_{1}^{\alpha_{1}} \cdots f_{r}^{\alpha_{r}} \in \operatorname{supp}(f) \text { para alguns } \alpha_{2}, \ldots, \alpha_{r} \in \mathbb{Z}\right\} \\
& =\min \left\{\alpha_{1}: a_{\left(\alpha_{1}, \ldots, \alpha_{r}\right)} \neq 0 \text { para alguns } \alpha_{2}, \ldots, \alpha_{r} \in \mathbb{Z}\right\}
\end{aligned}
$$

e $\hat{\nu}(0)=\infty$.

Lema 3.1.2. $\hat{\nu}: K((G)) \longrightarrow \mathbb{Z} \cup\{\infty\}$ é uma valorização de $K((G))$ que estende $\nu: Q(K G) \longrightarrow$ $\mathbb{Z} \cup\{\infty\}$.

Demonstração: Para mostrar que $\hat{\nu}$ é uma valorização basta proceder de maneira análoga ao que foi feito para $o_{f_{1}}$ na prova do Lema 3.1.1. Como $\mu:=\left.\hat{\nu}\right|_{Q(K G)}$ é uma valorização de $Q(K G)$ e $\left.\mu\right|_{K G}=o_{f_{1}}$, então $\mu=\nu$, ou seja, $\left.\hat{\nu}\right|_{Q(K G)}=\nu$, pela Proposição 1.3.4.

Seja $\hat{d}$ a métrica sobre $K((G))$ definida por:

$$
\hat{d}(f, g)=c^{\hat{\nu}(f-g)}, f, g \in K((G)) .
$$

Lema 3.1.3. $(K((G)), \hat{d})$ é um espaço métrico completo.

Demonstração: Seja $\left(u_{n}\right)$ uma seqüência de Cauchy em $K((G))$,

$$
u_{n}=\sum_{I=\left(\alpha_{1}, \ldots, \alpha_{r}\right)} a_{I}^{(n)} f_{1}^{\alpha_{1}} f_{2}^{\alpha_{2}} \cdots f_{r}^{\alpha_{r}}, a_{I}^{(n)} \in K
$$

Para todo $k \in \mathbb{Z}$ existe $n_{k} \in \mathbb{N}$ tal que $\hat{d}\left(u_{p}, u_{q}\right)<c^{k}$, se $p, q \geq n_{k}$. Se $\eta \in \mathbb{N}$ é tal que $\eta>$ $\max \left\{n_{0}, n_{1}\right\}$ então $\hat{d}\left(u_{p}, u_{q}\right)<c^{1}=c$, para todo $p, q \geq \eta$. Logo $\eta$ é um número natural tal que $n_{0}<\eta$ e $\hat{d}\left(u_{p}, u_{q}\right)<c^{1}$, para todo $p, q \geq \eta$. Podemos então supor que $n_{0}<n_{1}$. É possível então tomar

$$
n_{0}<n_{1}<n_{2}<\cdots<n_{k}<\cdots \quad(k>0) .
$$

Observe que, para todo $k \in \mathbb{Z}$,

$$
\hat{d}\left(u_{p}, u_{q}\right)<c^{k} \Leftrightarrow a_{I}^{(p)}=a_{I}^{(q)}, \forall I=\left(\alpha_{1}, \ldots, \alpha_{r}\right) \operatorname{com} \alpha_{1} \leq k
$$


De fato,

$$
\begin{aligned}
c^{k}>\hat{d}\left(u_{p}, u_{q}\right)=c^{\hat{\nu}\left(u_{p}-u_{q}\right)} & \Leftrightarrow k<\hat{\nu}\left(u_{p}-u_{q}\right)=\hat{\nu}\left(\sum\left(a_{I}^{(p)}-a_{I}^{(q)}\right) f_{1}^{\alpha_{1}} f_{2}^{\alpha_{2}} \cdots f_{r}^{\alpha_{r}}\right) \\
& \Leftrightarrow a_{I}^{(p)}-a_{I}^{(q)}=0, \forall I=\left(\alpha_{1}, \ldots, \alpha_{r}\right) \operatorname{com} \alpha_{1} \leq k .
\end{aligned}
$$

Considere a subseqüência $\left(u_{n_{k}}\right)_{k \geq 0}$ de $\left(u_{n}\right)$. Então $u_{n_{k}}=\sum_{I} a_{I}^{\left(n_{k}\right)} f_{1}^{\alpha_{1}} f_{2}^{\alpha_{2}} \cdots f_{r}^{\alpha_{r}}, k \geq 0$. Se $l \geq k \geq 0$ então $n_{l} \geq n_{k} \geq n_{0}$. Segue de (3.1) que,

$$
\begin{aligned}
& a_{I}^{\left(n_{k}\right)}=a_{I}^{\left(n_{0}\right)}, \forall I=\left(\alpha_{1}, \ldots, \alpha_{r}\right) \operatorname{com} \alpha_{1} \leq 0, \\
& a_{I}^{\left(n_{l}\right)}=a_{I}^{\left(n_{k}\right)}, \forall I=\left(\alpha_{1}, \ldots, \alpha_{r}\right) \operatorname{com} \alpha_{1} \leq k,
\end{aligned}
$$

para todo $l \geq k \geq 0$. Defina

$$
u=\sum_{\substack{I=\left(\alpha_{1}, \ldots, \alpha_{r}\right) \\ \alpha_{1}<0}} a_{I}^{\left(n_{0}\right)} f_{1}^{\alpha_{1}} f_{2}^{\alpha_{2}} \cdots f_{r}^{\alpha_{r}}+\sum_{\substack{I=\left(\alpha_{1}, \ldots, \alpha_{r}\right) \\ \alpha_{1} \geq 0}} a_{I}^{\left(n_{\alpha_{1}}\right)} f_{1}^{\alpha_{1}} f_{2}^{\alpha_{2}} \cdots f_{r}^{\alpha_{r}} .
$$

Mostremos que $u \in K((G))$, ou seja, que $\operatorname{supp}(u)$ é bem-ordenado. Vamos denotar por $v$ a primeira parcela da soma em $u$ e por $w$ a segunda parcela. Como $\operatorname{supp}(v) \subset \operatorname{supp}\left(u_{n_{0}}\right)$ então $\operatorname{supp}(v)$ é bemordenado. Fixe $\alpha_{1} \geq 0$ tal que $f_{1}^{\alpha_{1}} f_{2}^{\alpha_{2}} \cdots f_{r}^{\alpha_{r}} \in \operatorname{supp}(w)$ para alguns $\alpha_{2}, \ldots, \alpha_{r} \in \mathbb{Z}$, e considere o conjunto

$$
N_{\alpha_{1}}=\left\{\alpha_{2} \in \mathbb{Z}: f_{1}^{\alpha_{1}} f_{2}^{\alpha_{2}} \cdots f_{r}^{\alpha_{r}} \in \operatorname{supp}(w) \text { para alguns } \alpha_{3}, \ldots, \alpha_{r}\right\} .
$$

Suponha que $N_{\alpha_{1}}$ não tenha elemento mínimo. Então existe uma seqüência decrescente de inteiros

$$
\alpha_{21}>\alpha_{22}>\alpha_{23}>\cdots>\alpha_{2 l}>\cdots
$$

tal que $f_{1}^{\alpha_{1}} f_{2}^{\alpha_{2 l}} f_{3}^{\alpha_{3 l}} \cdots f_{r}^{\alpha_{r l}} \in \operatorname{supp}(w)$ para alguns $\alpha_{3 l}, \ldots, \alpha_{r l} \in \mathbb{Z}$, para todo $l \geq 1$, ou seja, $a_{\left(\alpha_{1}, \alpha_{2 l}, \alpha_{3 l}, \ldots, \alpha_{r l}\right)}^{\left(n_{\alpha_{1}}\right)} \neq 0$, para todo $l \geq 1$. Assim, $f_{1}^{\alpha_{1}} f_{2}^{\alpha_{2 l}} f_{3}^{\alpha_{3 l}} \cdots f_{r}^{\alpha_{r l}} \in \operatorname{supp}\left(u_{n_{\alpha_{1}}}\right)$, para todo $l \geq 1$, e portanto $\left\{f_{1}^{\alpha_{1}} f_{2}^{\alpha_{2 l}} \cdots f_{r}^{\alpha_{r l}}: l \geq 1\right\} \subset \operatorname{supp}\left(u_{n_{\alpha_{1}}}\right)$ é um subconjunto que não possui elemento mínimo uma vez que $f_{1}^{\alpha_{1}} f_{2}^{\alpha_{21}} \cdots f_{r}^{\alpha_{r 1}}>f_{1}^{\alpha_{1}} f_{2}^{\alpha_{22}} \cdots f_{r}^{\alpha_{r 2}}>\cdots>f_{1}^{\alpha_{1}} f_{2}^{\alpha_{2 l}} \cdots f_{r}^{\alpha_{r l}}>\cdots$. Isso é um absurdo pois $u_{n_{\alpha_{1}}} \in K((G))$ e portanto seu suporte é bem-ordenado. Logo $N_{\alpha_{1}}$ possui elemento mínimo.

Agora fixe $\alpha_{1} \geq 0$ e $\alpha_{2}, \ldots, \alpha_{n} \in \mathbb{Z}, 2 \leq n<r$, tais que $f_{1}^{\alpha_{1}} f_{2}^{\alpha_{2}} f_{n}^{\alpha_{n}} f_{n+1}^{\alpha_{n+1}} \cdots f_{r}^{\alpha_{r}} \in \operatorname{supp}(w)$ para 
alguns $\alpha_{n+1}, \ldots, \alpha_{r} \in \mathbb{Z}$, e considere o conjunto

$$
N_{\left(\alpha_{1}, \ldots, \alpha_{n}\right)}=\left\{\alpha_{n+1} \in \mathbb{Z}: f_{1}^{\alpha_{1}} f_{2}^{\alpha_{2}} \cdots f_{r}^{\alpha_{r}} \in \operatorname{supp}(w) \text { para alguns } \alpha_{n+2}, \ldots, \alpha_{r} \in \mathbb{Z}\right\} .
$$

$\left(\right.$ Se $\left.n=r-1, N_{\left(\alpha_{1}, \ldots, \alpha_{r-1}\right)}=\left\{\alpha_{r} \in \mathbb{Z}: f_{1}^{\alpha_{1}} f_{2}^{\alpha_{2}} \cdots f_{r}^{\alpha_{r}} \in \operatorname{supp}(w)\right\}\right)$. Suponha que $N_{\left(\alpha_{1}, \ldots, \alpha_{n}\right)}$ não tenha elemento mínimo e seja

$$
\alpha_{n+1,1}>\alpha_{n+1,2}>\alpha_{n+1,3}>\cdots>\alpha_{n+1, l}>\cdots
$$

uma seqüência decrescente em $N_{\left(\alpha_{1}, \ldots, \alpha_{n}\right)}$. Então, para todo $l \geq 1$, existem $\alpha_{n+2, l}, \ldots, \alpha_{r l} \in \mathbb{Z}$ tais que $f_{1}^{\alpha_{1}} \cdots f_{n}^{\alpha_{n}} f_{n+1}^{\alpha_{n+1, l}} f_{n+2}^{\alpha_{n+2, l}} \cdots f_{r}^{\alpha_{r l}} \in \operatorname{supp}(w)$, para todo $l \geq 1$, ou seja, $a_{\left(\alpha_{1}, \ldots, \alpha_{n}, \alpha_{n+1, l}, \ldots, \alpha_{r l}\right)}^{\left(n_{\alpha_{1}}\right)} \neq 0$, para todo $l \geq 1$. Logo $\left\{f_{1}^{\alpha_{1}} \cdots f_{n}^{\alpha_{n}} f_{n+1}^{\alpha_{n+1, l}} \cdots f_{r}^{\alpha_{r l}}: l \geq 1\right\} \subset \operatorname{supp}\left(u_{n_{\alpha_{1}}}\right)$ é um subconjunto que não possui elemento mínimo pois $f_{1}^{\alpha_{1}} \cdots f_{n}^{\alpha_{n}} f_{n+1}^{\alpha_{n+1,1}} \cdots f_{r}^{\alpha_{r 1}}>f_{1}^{\alpha_{1}} \cdots f_{n}^{\alpha_{n}} f_{n+1}^{\alpha_{n+1,2}} \cdots f_{r}^{\alpha_{r 2}}>\cdots$. Isso é um absurdo pois $u_{n_{\alpha_{1}}}$ tem suporte bem-ordenado. Logo $N_{\left(\alpha_{1}, \ldots, \alpha_{n}\right)}$ possui elemento mínimo, para todo $1 \leq n<r$.

Seja $S \subset \operatorname{supp}(w)$ um subconjunto não-vazio e defina os seguintes inteiros:

$$
\begin{gathered}
\alpha_{1 S}=\min \left\{\alpha_{1} \geq 0: f_{1}^{\alpha_{1}} f_{2}^{\alpha_{2}} \cdots f_{r}^{\alpha_{r}} \in S \text { para alguns } \alpha_{2}, \ldots, \alpha_{r} \in \mathbb{Z}\right\}, \\
\alpha_{n S}=\min \left\{\alpha_{n} \in \mathbb{Z}: f_{1}^{\alpha_{1 S}} \cdots f_{n-1}^{\alpha_{n-1, S}} f_{n}^{\alpha_{n}} \cdots f_{r}^{\alpha_{r}} \in S \text { para alguns } \alpha_{n+1}, \ldots, \alpha_{r} \in \mathbb{Z}\right\} \\
\subset N_{\left(\alpha_{1 S}, \ldots, \alpha_{n-1, S}\right)}, 1<n<r, \\
\alpha_{r S}=\min \left\{\alpha_{r} \in \mathbb{Z}: f_{1}^{\alpha_{1 S}} \cdots f_{r-1}^{\alpha_{r-1, S}} f_{r}^{\alpha_{r}} \in S\right\} \subset N_{\left(\alpha_{1 S}, \ldots, \alpha_{r-1, S}\right)},
\end{gathered}
$$

que existem pelo Princípio da Boa Ordem. Então $g=f_{1}^{\alpha_{1 S}} f_{2}^{\alpha_{2 S}} \cdots f_{r}^{\alpha_{r S}}$ é o elemento mínimo de $S$. De fato, seja $h=f_{1}^{\beta_{1}} f_{2}^{\beta_{2}} \cdots f_{r}^{\beta_{r}} \in S$. Então $\beta_{1} \geq \alpha_{1 S}$. Se $\beta_{1}>\alpha_{1 S}$ então $h>g$. Suponha que $\beta_{1}=\alpha_{1 S}$ e seja $m=\max \left\{i \geq 1: \beta_{1}=\alpha_{1 S}, \ldots, \beta_{i}=\alpha_{i s}\right\}$. Se $m=r$ então $h=g$. Se $m<r$ então $h=f_{1}^{\alpha_{1 S}} \cdots f_{m}^{\alpha_{m S}} f_{m+1}^{\beta_{m+1}} \cdots f_{r}^{\beta_{r}} \operatorname{com} \beta_{m+1} \neq \alpha_{m+1, S}$. Como $h \in S$ então

$$
\begin{aligned}
\beta_{m+1} & \geq \min \left\{\alpha_{m+1} \in \mathbb{Z}: f_{1}^{\alpha_{1 S}} \cdots f_{m}^{\alpha_{m} S} f_{m+1}^{\alpha_{m+1}} \cdots f_{r}^{\alpha_{r}} \in S \text { para alguns } \alpha_{m+2}, \ldots, \alpha_{r} \in \mathbb{Z}\right\} \\
& =\alpha_{m+1, S}
\end{aligned}
$$

e $\operatorname{assim} h \geq g$, para todo $h \in S$. Logo $g=\min S$ e portanto $\operatorname{supp}(w)$ é bem-ordenado. Segue que 
$u=v+w \in K((G))$.

Mostremos agora que $u_{n} \rightarrow u$. Dado $\epsilon>0$ seja $t$ um inteiro positivo tal que $c^{t}<\epsilon$. Se $k \geq t$ então $c^{k} \leq c^{t}<\epsilon$. Temos

$$
\begin{aligned}
u_{n_{k}}-u= & \sum_{\substack{I=\left(\alpha_{1}, \ldots, \alpha_{r}\right) \\
\alpha_{1}<0}}\left(a_{I}^{\left(n_{k}\right)}-a_{I}^{\left(n_{0}\right)}\right) f_{1}^{\alpha_{1}} f_{2}^{\alpha_{2}} \cdots f_{r}^{\alpha_{r}}+\sum_{\substack{I=\left(\alpha_{1}, \ldots, \alpha_{r}\right) \\
0 \leq \alpha_{1} \leq k}}\left(a_{I}^{\left(n_{k}\right)}-a_{I}^{\left(n_{\alpha_{1}}\right)}\right) f_{1}^{\alpha_{1}} f_{2}^{\alpha_{2}} \cdots f_{r}^{\alpha_{r}} \\
& +\sum_{\substack{I=\left(\alpha_{1}, \ldots, \alpha_{r}\right) \\
\alpha_{1} \geq k+1}}\left(a_{I}^{\left(n_{k}\right)}-a_{I}^{\left(n_{\alpha_{1}}\right)}\right) f_{1}^{\alpha_{1}} f_{2}^{\alpha_{2}} \cdots f_{r}^{\alpha_{r}} \\
= & \sum_{\substack{I=\left(\alpha_{1}, \ldots, \alpha_{r}\right) \\
\alpha_{1} \geq k+1}}\left(a_{I}^{\left(n_{k}\right)}-a_{I}^{\left(n_{\alpha_{1}}\right)}\right) f_{1}^{\alpha_{1}} f_{2}^{\alpha_{2}} \cdots f_{r}^{\alpha_{r}},
\end{aligned}
$$

por (3.2) e (3.3). Então $\hat{\nu}\left(u_{n_{k}}-u\right) \geq k+1>k$ e portanto $\hat{d}\left(u_{n_{k}}, u\right)=c^{\hat{\nu}\left(u_{n_{k}}-u\right)}<c^{k}<\epsilon$. Portanto a subseqüência $\left(u_{n_{k}}\right)$ de $\left(u_{n}\right)$ converge para $u$. Como $\left(u_{n}\right)$ é de Cauchy então $u_{n} \rightarrow u$ e portanto $K((G))$ é completo.

\subsection{Corpos livres em $K((G))$}

Nesta seção usaremos o Teorema 2.2.4 para mostrar que o corpo das séries de Malcev-Neumann $K((G))$ contém um corpo livre sobre o centro de $Q(K G)$. Primeiramente, vamos encontrar o centro de $Q(K G)$. Para isso, vamos utilizar o seguinte resultado:

Teorema 3.2.1 ([44], pg.332). Se G é nilpotente, finitamente gerado, livre de torção e $K$ é um corpo comutativo então

$$
Z(Q(K G))=Q(K \mathcal{Z}(G))
$$

Vamos considerar uma $\mathcal{F}$-série de $G$

$$
G=F_{1} \supset F_{2} \supset \cdots \supset F_{r} \supset F_{r+1}=\{1\}
$$

que seja um refinamento da série central superior de $G$, como na demonstração da Proposição 1.4.7.

Lema 3.2.2. $Z(Q(K G))=K\left(f_{j}, \ldots, f_{r}\right)$, onde $F_{j}=\mathcal{Z}(G)$.

Demonstração: Como $G$ é não-abeliano então $f_{1} \notin \mathcal{Z}(G)$ pois, caso contrário, $f_{1} \in \mathcal{Z}(G) \subset F_{2} \Rightarrow$ 
$F_{1} / F_{2}=\left\langle f_{1} F_{2}\right\rangle=\{1\}$, o que é uma contradição já que $F_{1} / F_{2}$ é um grupo cíclico infinito. Seja $j>1$ tal que $F_{j}=\mathcal{Z}(G)$. Então

$$
\mathcal{Z}(G)=F_{j} \supset F_{j+1} \supset \cdots \supset F_{r} \supset F_{r+1}=\{1\}
$$

é uma $\mathcal{F}$-série para $\mathcal{Z}(G)$. Logo todo elemento de $\mathcal{Z}(G)$ é escrito de maneira única na forma $g=$ $f_{j}^{\alpha_{j}} \cdots f_{r}^{\alpha_{r}}$ e portanto

$$
K \mathcal{Z}(G)=\left\{\sum_{I} \alpha_{I} f_{j}^{\alpha_{j}} \cdots f_{r}^{\alpha_{r}}: I=\left(\alpha_{j}, \ldots, \alpha_{r}\right) \in \mathbb{Z}^{r-j+1}\right\} .
$$

Como $\left\{f_{j}^{\alpha_{j}} \cdots f_{r}^{\alpha_{r}}: \alpha_{i} \in \mathbb{Z}\right\}$ é linearmente independente sobre $K$ então

$$
K \mathcal{Z}(G)=K\left[f_{j}, f_{j}^{-1}, \ldots, f_{r}, f_{r}^{-1}\right]
$$

o anel dos polinômios de Laurent nas indeterminadas centrais $f_{j}, \ldots, f_{r}$ sobre $K$. Como

$$
K\left[f_{j}, \ldots, f_{r}\right] \subset K\left[f_{j}, f_{j}^{-1}, \ldots, f_{r}, f_{r}^{-1}\right] \subset K\left(f_{j}, \ldots, f_{r}\right)
$$

onde $K\left(f_{j}, \ldots, f_{r}\right)$ é o corpo das funções racionais em $f_{j}, \ldots, f_{r}$ sobre $K$, segue que $Q(K \mathcal{Z}(G))=$ $Q\left(K\left[f_{j}, f_{j}^{-1}, \ldots, f_{r}, f_{r}^{-1}\right]\right)=K\left(f_{j}, \ldots, f_{r}\right)$. Logo, pelo teorema acima, $Z(Q(K G))=K\left(f_{j}, \ldots, f_{r}\right)$.

Teorema 3.2.3. Seja $K$ um corpo comutativo e seja $G$ um grupo nilpotente, finitamente gerado, livre de torção, não-abeliano. Então $K((G))$ contém um corpo livre $Z \nless X \ngtr$, onde $Z$ é o centro de $Q(K G)$ e $X$ é um conjunto enumerável.

Demonstração: Já sabemos que $Z(Q(K G))=K\left(f_{j}, \ldots, f_{r}\right)$, que é um corpo infinito. Mostremos que $Q(K G)$ tem dimensão infinita sobre $K\left(f_{j}, \ldots, f_{r}\right){ }^{1}$ Para isso, considere o conjunto $\left\{f_{1}^{i}: i \geq\right.$ 1\} $\subset K G$ e sejam $g_{1} h_{1}^{-1}, g_{2} h_{2}^{-1}, \ldots, g_{n} h_{n}^{-1} \in K\left(f_{j}, \ldots, f_{r}\right), g_{i}, h_{i} \in K\left[f_{j}, \ldots, f_{r}\right], h_{i} \neq 0$, tais que $\left(g_{1} h_{1}^{-1}\right) f_{1}+\left(g_{2} h_{2}^{-1}\right) f_{1}^{2}+\cdots+\left(g_{n} h_{n}^{-1}\right) f_{1}^{n}=0$. Seja $h \in K\left[f_{j}, \ldots, f_{r}\right]$ tal que $g_{i} h_{i}^{-1}=k_{i} h^{-1}$, para algum $k_{i} \in K\left[f_{j}, \ldots, f_{r}\right]$, para todo $i=1, \ldots, n$. Então $\left(k_{1} h^{-1}\right) f_{1}+\left(k_{2} h^{-1}\right) f_{1}^{2}+\cdots+\left(k_{n} h^{-1}\right) f_{1}^{n}=0$

\footnotetext{
${ }^{1}$ Vale dizer que já sabemos de [30] que $Q(K G)$ tem dimensão infinita sobre seu centro, uma vez que $Q(K G)$ contém uma álgebra livre. Mas, uma vez que o cálculo da dimensão é simples, faremos uma demonstração direta.
} 
e portanto $k_{1} f_{1}+k_{2} f_{1}^{2}+\cdots+k_{n} f_{1}^{n}=0$. Escrevamos $k_{i}=\sum_{I} a_{I}^{(i)} f_{j}^{\alpha_{j}} \cdots f_{r}^{\alpha_{r}} \in K\left[f_{j}, \ldots, f_{r}\right]$. Então

$$
\begin{aligned}
0 & =\sum_{I} a_{I}^{(1)} f_{j}^{\alpha_{j}} \cdots f_{r}^{\alpha_{r}} f_{1}+\cdots+\sum_{I} a_{I}^{(n)} f_{j}^{\alpha_{j}} \cdots f_{r}^{\alpha_{r}} f_{1}^{n} \\
& =\sum_{I} a_{I}^{(1)} f_{1} f_{j}^{\alpha_{j}} \cdots f_{r}^{\alpha_{r}}+\cdots+\sum_{I} a_{I}^{(n)} f_{1}^{n} f_{j}^{\alpha_{j}} \cdots f_{r}^{\alpha_{r}} \\
& \Rightarrow a_{I}^{(i)}=0, \forall I=\left(\alpha_{j}, \ldots, \alpha_{r}\right), \forall i \Rightarrow k_{i}=0, \forall i \Rightarrow g_{i} h_{i}^{-1}=0, \forall i .
\end{aligned}
$$

Logo $\left\{f_{1}^{i}: i \geq 1\right\} \subset K G$ é linearmente independente sobre $K\left(f_{j}, \ldots, f_{r}\right)$ e, portanto, $Q(K G)$ tem dimensão infinita sobre $K\left(f_{j}, \ldots, f_{r}\right)$. Se $\nu$ é a única valorização de $Q(K G)$ tal que $\left.\nu\right|_{K G}=o_{f_{1}}$, a função ordem com relação a $f_{1}$, então $\nu$ é uma valorização discreta e portanto o completamento de $Q(K G)$ com relação à topologia definida por $\nu$ contém um corpo livre $Z \nless X \ngtr$, sobre um conjunto enumerável $X$.

A inclusão $f: Q(K G) \longrightarrow K((G))$ é um mergulho isométrico, ou seja,

$$
\hat{d}(f(x), f(y))=c^{\hat{\nu}(f(x)-f(y))}=c^{\hat{\nu}(x-y)}=c^{\nu(x-y)}=d(x, y)
$$

para todos $x, y \in Q(K G)$, pelo Lema 3.1.2. Como $K((G))$ é completo então o fecho $\overline{f(Q(K G))}$ do subespaço $f(Q(K G))$ do espaço métrico $K((G))$ é completo e, portanto, $\overline{f(Q(K G))}$ é o completamento de $Q(K G)$. Portanto $K((G))$ contém $Z \nless X \ngtr$.

Vale observar que o Teorema 3.2.3 continua valendo se $K$ não for comutativo. De fato, $K G$ é um domínio noetheriano, pela Proposição 1.6 de [35, pg. 7], e todos os resultados da Seção 3.1 são válidos para $K$ não comutativo, com as mesmas demonstrações. Como $\left\{f_{r}{ }^{i}: i \in \mathbb{Z}\right\} \subset Z=Z(Q(K G))$ então $Z$ é infinito. Se $C=Z(K)$ então, pelo Corolário pg. 47 de [30], $Q(C G)$ contém uma $C$-álgebra livre e, portanto, $Q(K G)$ também contém. Logo a dimensão de $Q(K G)$ sobre $Z$ é infinita (uma vez que toda álgebra de dimensão finita $n$ satisfaz o polinômio standard $S_{n+1}$ e, portanto, não pode conter uma álgebra livre). Logo $\widehat{Q(K G)}$ contém um corpo livre $Z \nless X \ngtr$ e, portanto, $K((G))$ também contém.

Se $H$ for um grupo residualmente-\{nilpotente, livre de torção\}, finitamente gerado, então $H$ é um grupo ordenável, pelo Lema 13.2.2 de [34]. As investigações feitas até o momento nos levam a acreditar que existe uma especialização, nas hipóteses do Teorema 2.3.1, de um corpo de séries de Malcev-Neumann $K((H))$ em $K((G))$, onde $G$ é um grupo nilpotente, finitamente gerado, livre de torção, com a relação de ordem definida na Seção 1.4, e $K$ é um corpo comutativo. Se essa suspeita 
for confirmada teremos então um corpo livre em $K((H))$, pelo Teorema 2.3.1.

\subsection{Corpos livres em $K((G, \sigma))$}

Consideremos agora o corpo das séries de Malcev-Neumann skew $K((G, \sigma))$, onde $\sigma: G \longrightarrow$ Aut $(K)$ é um homomorfismo. Uma vez que $K(G, \sigma) \subset K((G, \sigma))$ então $K(G, \sigma)$ é um domínio. Além disso, segue da Proposição 1.6 de [35, pg. 7] que $K(G, \sigma)$ é um anel noetheriano à direita. Em particular, $K(G, \sigma)$ é um domínio de Ore à direita e seu corpo clássico de frações será denotado por $Q(K(G, \sigma))$. Temos então as inclusões $K(G, \sigma) \subset Q(K(G, \sigma)) \subset K((G, \sigma))$.

Assim como no caso das séries de Malcev-Neumann $K((G))$, também conseguimos encontrar casos em que $K((G, \sigma))$ contém um corpo livre. Considere a função $o_{f_{1}}: K(G, \sigma) \longrightarrow \mathbb{Z} \cup\{\infty\}$ definida de forma análoga ao caso $\sigma=1$. Do mesmo modo, mostra-se que $o_{f_{1}}$ é uma valorização de $K(G, \sigma)$. Seja $\nu: Q(K(G, \sigma)) \longrightarrow \mathbb{Z} \cup\{\infty\}$ a única valorização de $Q(K G)$ tal que $\left.\nu\right|_{K(G, \sigma)}=o_{f_{1}}$. Também definida de forma análoga ao caso $\sigma=1$, considere a função $\hat{\nu}: K((G, \sigma)) \longrightarrow \mathbb{Z} \cup\{\infty\}$, que é uma valorização de $K((G, \sigma))$. Como $\mu:=\left.\hat{\nu}\right|_{Q(K(G, \sigma))}$ é uma valorização de $Q(K(G, \sigma))$ e $\left.\mu\right|_{K(G, \sigma)}=o_{f_{1}}$, então $\left.\hat{\nu}\right|_{Q(K(G, \sigma))}=\nu$, pela Proposição 1.3.4. Sejam $d$ e $\hat{d}$ as métricas definidas sobre $Q(K(G, \sigma))$ e $K((G, \sigma))$, respectivamente, como no caso $\sigma=1$. Prosseguindo de maneira análoga ao que fizemos na Seção 3.1 é possível mostrar que $(K((G, \sigma)), \hat{d})$ é um espaço métrico completo.

No caso em que $\sigma \neq 1$ não temos um resultado que nos ajude a calcular o centro de $Q(K(G, \sigma))$, como tínhamos o Teorema 3.2.1 no caso $\sigma=1 \mathrm{e}$, portanto, não é possível afirmar se $Q(K(G, \sigma))$ tem dimensão infinita sobre seu centro e se seu centro é infinito. No entanto, com algumas hipóteses sobre $\sigma$ ainda é possível garantir que $K((G, \sigma))$ contenha um corpo livre. Por exemplo, temos o seguinte

Teorema 3.3.1. Seja $K$ um corpo comutativo e seja $G$ um grupo nilpotente, finitamente gerado, livre de torção, não-abeliano. Se $\sigma: G \longrightarrow \operatorname{Aut}(K)$ for um homomorfismo injetor tal que o corpo $K^{G}=\left\{a \in K: \sigma_{g}(a)=a, \forall g \in G\right\}$ seja infinito então $K((G, \sigma))$ contém um corpo livre $K^{G} \nless X \ngtr$ sobre um conjunto enumerável $X$.

A demonstração deste teorema segue o roteiro descrito acima e faz uso da seguinte caracterização do centro de $K((G, \sigma))$ :

Teorema 3.3.2 ([18], Corolário 14.26). Se $R$ é um corpo comutativo, $G$ é um grupo ordenado e 
$\omega: G \longrightarrow \operatorname{Aut}(R)$ é um homomorfismo injetor, então

$$
Z(R((G, \omega)))=R^{G}:=\left\{r \in R: \omega_{g}(r)=r, \forall g \in G\right\}
$$

e $R((G, \omega))$ tem dimensão infinita sobre $R^{G}$. 


\section{Capítulo 4}

\section{Séries de Laurent}

Neste capítulo usaremos o Teorema 2.2.4 para provar a existência de corpos livres em alguns corpos de séries de Laurent skew, como, por exemplo, o completamento do corpo de Weyl e do corpo das matrizes quânticas $2 \times 2$.

\subsection{Séries de Laurent skew}

Sejam $K$ um corpo, $\alpha$ um automorfismo de $K$ e $\delta$ uma $\alpha$-derivação não-nula de $K$. Considere o corpo $K((y ; \alpha, \delta))$, onde $y a=\alpha(a) y+y \delta(a) y, a \in K$, como no Teorema 1.6.6. Defina sobre $K((y ; \alpha, \delta))$ uma função $\omega: K((y ; \alpha, \delta)) \longrightarrow \mathbb{Z} \cup\{\infty\}$ da seguinte forma: se $f \in K((y ; \alpha, \delta))$,

$$
\omega(f)=\sup \left\{n: f \in K[[y ; \alpha, \delta]] y^{n}=y^{n} K[[y ; \alpha, \delta]]\right\} .
$$

Lema 4.1.1. $\omega$ é uma valorização de $K((y ; \alpha, \delta))$.

Demonstração: É óbvio que $\omega(1)=0$ e $\omega(0)=\infty$. Sejam $f=\sum_{i=k}^{\infty} a_{i} y^{i}, g=\sum_{i=l}^{\infty} b_{i} y^{i}$ com $a_{k}, b_{l} \neq 0$. Suponha sem perda de generalidade que $k \leq l$. Então $f+g=\sum_{i=k}^{\infty}\left(a_{i}+b_{i}\right) y^{i}$, onde $b_{i}=0$ se $i<l$. Se $a_{k}+b_{k} \neq 0$ então $\omega(f+g)=k=\min \{k, l\}=\min \{\omega(f), \omega(g)\}$. Se $a_{k}+b_{k}=0$ então $\omega(f+g)>k=\min \{\omega(f), \omega(g)\}$. Portanto $\omega(f+g) \geq \min \{\omega(f), \omega(g)\}$. Se $f=0$ ou $g=0$ 
então $\omega(f+g)=\min \{\omega(f), \omega(g)\}$. Agora, sejam $f=\sum_{i=-k}^{\infty} a_{i} y^{i}, g=\sum_{j=-l}^{\infty} b_{j} y^{j}, k, l \geq 0$. Então

$$
\begin{aligned}
f g & =\sum_{i=-k}^{\infty} \sum_{j=-l}^{\infty} a_{i} y^{i} b_{j} y^{j}=\sum_{i \leq 0} \sum_{j=-l}^{\infty} a_{i} y^{i} b_{j} y^{j}+\sum_{i>0} \sum_{j=-l}^{\infty} a_{i} y^{i} b_{j} y^{j} \\
& =\sum_{i \leq 0} \sum_{j=-l}^{\infty} a_{i} x^{-i} b_{j} y^{j}+\sum_{i>0} \sum_{j=-l}^{\infty} a_{i}\left(\sum_{n_{1}, \ldots, n_{i} \geq 0} \alpha \delta^{n_{1}} \cdots \alpha \delta^{n_{i}}\left(b_{j}\right) y^{n_{1}+\cdots+n_{i}+i}\right) y^{j}, \text { por } \\
& =\sum_{i=0}^{k} \sum_{j=-l}^{\infty} a_{-i} x^{i} b_{j} y^{j}+\sum_{i=1}^{\infty} \sum_{j=-l}^{\infty} \sum_{n_{1}, \ldots, n_{i} \geq 0} a_{i} \alpha \delta^{n_{1}} \cdots \alpha \delta^{n_{i}}\left(b_{j}\right) y^{n_{1}+\cdots+n_{i}+i+j}
\end{aligned}
$$

Agora,

$$
\begin{aligned}
\sum_{i=0}^{k} \sum_{j=-l}^{\infty} a_{-i} x^{i} b_{j} y^{j} & =\sum_{i=0}^{k} \sum_{j=-l}^{\infty} a_{-i}\left(\sum_{m=0}^{i}(-1)^{i-m} g^{\prime}(m, i-m)\left(b_{j}\right) x^{m}\right) y^{j}, \text { por }( \\
& =\sum_{i=0}^{k} \sum_{j=-l}^{\infty}\left(\sum_{m=0}^{i}(-1)^{i-m} a_{-i} g^{\prime}(m, i-m)\left(b_{j}\right) y^{j-m}\right)
\end{aligned}
$$

e, portanto,

$$
\begin{aligned}
f g= & \sum_{i=0}^{k} \sum_{j=-l}^{\infty}\left(\sum_{m=0}^{i}(-1)^{i-m} a_{-i} g^{\prime}(m, i-m)\left(b_{j}\right) y^{j-m}\right) \\
& +\sum_{i=1}^{\infty} \sum_{j=-l}^{\infty} \sum_{n_{1}, \ldots, n_{i} \geq 0} a_{i} \alpha \delta^{n_{1}} \cdots \alpha \delta^{n_{i}}\left(b_{j}\right) y^{n_{1}+\cdots+n_{i}+i+j}
\end{aligned}
$$

Denotemos por $A$ a primeira parcela da soma acima e por $B$ a segunda parcela. Sejam $p$ e $q$ os menores índices tais que $a_{p} \neq 0$ e $b_{q} \neq 0$. Então $\omega(f)=p$ e $\omega(g)=q$. Vamos considerar dois casos:

1ํㅡ caso: $p \leq 0$.

Neste caso, $A=\sum_{i=0}^{-p} \sum_{j=q}^{\infty}\left(\sum_{m=0}^{i}(-1)^{i-m} a_{-i} g^{\prime}(m, i-m)\left(b_{j}\right) y^{j-m}\right)$ e portanto a menor potência possível de $y$ em $A$ é $p+q$, que ocorre quando $i=m=-p$ e $j=q$. Tomando esses valores para $i, j$ e $m$ temos $a_{-i} g^{\prime}(m, i-m)\left(b_{j}\right)=a_{p} g^{\prime}(-p, 0)\left(b_{q}\right)=a_{p}\left(\alpha^{-1}\right)^{-p}\left(b_{q}\right)=$ $a_{p} \alpha^{p}\left(b_{q}\right) \neq 0$. Além disso, $B=\sum_{i=1}^{\infty} \sum_{j=q}^{\infty} \sum_{n_{1}, \ldots, n_{i} \geq 0} a_{i} \alpha \delta^{n_{1}} \cdots \alpha \delta^{n_{i}}\left(b_{j}\right) y^{n_{1}+\cdots+n_{i}+i+j}$ e portanto as potências de $y$ que aparecem em $B$ são maiores ou iguais a $q+1$. Como $p \leq 0$ então $p+q<q+1$ e, portanto, $\omega(f g)=p+q$. 
$2^{0}$ caso: $p>0$.

Neste caso, $A=0$ e $B=\sum_{i=p}^{\infty} \sum_{j=q}^{\infty} \sum_{n_{1}, \ldots, n_{i} \geq 0} a_{i} \alpha \delta^{n_{1}} \cdots \alpha \delta^{n_{i}}\left(b_{j}\right) y^{n_{1}+\cdots+n_{i}+i+j}$. Então a menor potência possível de $y$ em $B$ é $p+q$, que ocorre quando $i=p, n_{1}=\cdots=n_{i}=0$ e $j=q$. Para esses valores temos $a_{i} \alpha \delta^{n_{1}} \cdots \alpha \delta^{n_{i}}\left(b_{j}\right)=a_{p} \alpha^{p}\left(b_{q}\right) \neq 0$ e, portanto, $\omega(f g)=p+q$.

Se $f=0$ ou $g=0$ é óbvio que $\omega(f g)=p+q$. Portanto $\omega$ é uma valorização de $K((y ; \alpha, \delta))$.

A função - deg : $K[x ; \alpha, \delta] \longrightarrow \mathbb{Z} \cup\{\infty\}$ (onde $y=x^{-1}$ ) é uma valorização e se estende de modo único a uma valorização $\nu$ sobre $K(x ; \alpha, \delta)$. Mais precisamente, se $f, g \in K[x ; \alpha, \delta], g \neq 0$, então

$$
\nu\left(f g^{-1}\right)=-\operatorname{deg}(f)-(-\operatorname{deg}(g))=\operatorname{deg}(g)-\operatorname{deg}(f) .
$$

É fácil ver que $\left.\omega\right|_{K(x ; \alpha, \delta)}=\nu$, por (1.7). Considere a métrica $d(u, v)=c^{\omega(u-v)}$ sobre $K((y ; \alpha, \delta))$, onde $c \in(0,1)$ é um número real fixo.

Lema 4.1.2. $K((y ; \alpha, \delta))$ é o completamento de $K(x ; \alpha, \delta)$.

Demonstração: Seja $u=\sum_{i=n}^{\infty} a_{i} y^{i} \in K((y ; \alpha, \delta)), a_{n} \neq 0$. Então $u=\left(\sum_{j=0}^{\infty} a_{n+j} y^{j}\right) y^{n}$. Considere a seqüência em $K(x ; \alpha, \delta)$ dada por $b_{m}=\left(\sum_{j=0}^{m} a_{n+j} x^{-j}\right) x^{-n}$. Dado $\epsilon>0$ seja $N$ um inteiro positivo tal que $c^{N+n+1}<\epsilon$. Então, se $m \geq N$,

$d\left(b_{m}, u\right)=c^{\omega\left(b_{m}-u\right)}=c^{\omega\left(\left(\sum_{j=m+1}^{\infty} a_{n+j} y^{j}\right) y^{n}\right)}=c^{\omega\left(\left(\sum_{j=m+1}^{\infty} a_{n+j} y^{j-m-1}\right) y^{n+m+1}\right)} \leq c^{n+m+1}<c^{n+N+1}<\epsilon$

e portanto $b_{m} \rightarrow u$. Logo $u \in \overline{K(x ; \alpha, \delta)}$ e portanto $\overline{K(x ; \alpha, \delta)}=K((y ; \alpha, \delta))$. Resta mostrar que $K((y ; \alpha, \delta))$ é completo. Seja $\left(u_{n}\right)$ uma seqüência de Cauchy em $K((y ; \alpha, \delta))$,

$$
u_{n}=\sum_{i \geq m_{n}} a_{i}^{(n)} y^{i}, a_{i}^{(n)} \in K, m_{n} \in \mathbb{Z}
$$

Para cada $k \in \mathbb{Z}$ seja $n_{k} \in \mathbb{N}$ tal que $d\left(u_{p}, u_{q}\right)<c^{k}$, se $p, q \geq n_{k}$. Se $\eta \in \mathbb{N}$ é tal que $\eta>\max \left\{n_{0}, n_{1}\right\}$ então, $d\left(u_{p}, u_{q}\right)<c^{1}=c$, para todo $p, q \geq \eta$. Logo $\eta$ é um número natural tal que $n_{0}<\eta \mathrm{e}$ $d\left(u_{p}, u_{q}\right)<c^{1}$, para todo $p, q \geq \eta$. Podemos então supor que, $n_{0}<n_{1}$. É possível então tomar

$$
n_{0}<n_{1}<n_{2}<\cdots<n_{k}<\cdots \quad(k>0) .
$$


Observe que, para todo $k \in \mathbb{Z}$,

$$
d\left(u_{p}, u_{q}\right)<c^{k} \Leftrightarrow a_{i}^{(p)}=a_{i}^{(q)}, \forall i \leq k
$$

De fato,

$$
c^{k}>d\left(u_{p}, u_{q}\right)=c^{\omega\left(u_{p}-u_{q}\right)} \Leftrightarrow k<\omega\left(u_{p}-u_{q}\right)=\omega\left(\sum\left(a_{i}^{(p)}-a_{i}^{(q)}\right) y^{i}\right) \Leftrightarrow a_{i}^{(p)}-a_{i}^{(q)}=0, \forall i \leq k .
$$

Considere a subseqüência $\left(u_{n_{k}}\right)_{k \geq 0}$ de $\left(u_{n}\right)$. Então $u_{n_{k}}=\sum_{i \geq m_{n_{k}}} a_{i}^{\left(n_{k}\right)} y^{i}, k \geq 0$. Se $l \geq k \geq 0$ então $n_{l} \geq n_{k} \geq n_{0}$. Segue de (4.2) que,

$$
\begin{aligned}
& a_{i}^{\left(n_{k}\right)}=a_{i}^{\left(n_{0}\right)}, \forall i \leq 0, \\
& a_{i}^{\left(n_{l}\right)}=a_{i}^{\left(n_{k}\right)}, \forall i \leq k,
\end{aligned}
$$

para todo $l \geq k \geq 0$. Defina

$$
u=\sum_{i<0} a_{i}^{\left(n_{0}\right)} y^{i}+\sum_{i \geq 0} a_{i}^{\left(n_{i}\right)} y^{i}
$$

Como $a_{i}^{\left(n_{0}\right)}=0$ para todo $i<m_{n_{0}}$, então a primeira parcela da soma em $u$ é finita (podendo, claro, ser nula) e, portanto, $u \in K((y ; \alpha, \delta))$. Mostremos que $u_{n} \rightarrow u$. Dado $\epsilon>0$ seja $t$ um inteiro positivo tal que $c^{t}<\epsilon$. Se $k \geq t$ então $c^{k} \leq c^{t}<\epsilon$. Temos

$$
\begin{aligned}
u_{n_{k}}-u & =\sum_{i<0}\left(a_{i}^{\left(n_{k}\right)}-a_{i}^{\left(n_{0}\right)}\right) y^{i}+\sum_{0 \leq i \leq k}\left(a_{i}^{\left(n_{k}\right)}-a_{i}^{\left(n_{i}\right)}\right) y^{i}+\sum_{i \geq k+1}\left(a_{i}^{\left(n_{k}\right)}-a_{i}^{\left(n_{i}\right)}\right) y^{i} \\
& =\sum_{i \geq k+1}\left(a_{i}^{\left(n_{k}\right)}-a_{i}^{\left(n_{i}\right)}\right) y^{i}
\end{aligned}
$$

por (4.3) e (4.4). Então $\omega\left(u_{n_{k}}-u\right) \geq k+1>k$ e portanto $d\left(u_{n_{k}}, u\right)=c^{\omega\left(u_{n_{k}}-u\right)}<c^{k}<\epsilon$. Portanto a subseqüência $\left(u_{n_{k}}\right)$ de $\left(u_{n}\right)$ converge para $u$. Como $\left(u_{n}\right)$ é de Cauchy então $u_{n} \rightarrow u$. Logo $K((y ; \alpha, \delta))$ é completo e, portanto, $K((y ; \alpha, \delta))$ é o completamento de $K(x ; \alpha, \delta)$.

Recordemos que o automorfismo $\alpha$ é dito ter ordem interna $r$ se $\alpha^{r}$ é a menor potência positiva de $\alpha$ a qual é um automorfismo interno. Se $\alpha^{r}$ não é interno para todo $r>0, \alpha$ é dito ter ordem interna infinita. O seguinte teorema será muito útil.

Teorema 4.1.3 ([8], Teorema 2.2.10). Sejam $D$ um anel com divisão com centro $C$, $\alpha$ um automorfismo de $D$ e $\delta$ uma $\alpha$-derivação. Considere o subcorpo $C_{0}=\{c \in C: \alpha(c)=c$ e $\delta(c)=0\}$, 
chamado o $(\alpha, \delta)$-centro reduzido de $D$. Se $D[x ; \alpha, \delta]$ for simples ou $\alpha$ tiver ordem interna infinita então $Z(D(x ; \alpha, \delta))=C_{0}$.

Temos então o seguinte resultado:

Teorema 4.1.4. Sejam $K$ um anel com divisão com centro $C, \alpha$ um automorfismo de $K$ e $\delta$ uma $\alpha$-derivação tal que o corpo $C_{0}=\{c \in C: \alpha(c)=c$ e $\delta(c)=0\}$ seja infinito. Se $K[x ; \alpha, \delta]$ for simples ou $\alpha$ tiver ordem interna infinita então o corpo das séries de Laurent $K((y ; \alpha, \delta)), y=x^{-1}$, contém um corpo livre $C_{0} \nless X \ngtr$, onde $X$ é um conjunto enumerável.

Demonstração: Sabemos que $Z(K(x ; \alpha, \delta))=C_{0}$, pelo Teorema 4.1.3. Como o conjunto $\left\{x^{i}: i \in\right.$ $\mathbb{Z}\} \subset K(x ; \alpha, \delta)$ é linearmente independente sobre $C_{0}$ (pois o é sobre $K$ ) então $\left[K(x ; \alpha, \delta): C_{0}\right]=$ $\infty$. Seja $\omega$ a valorização de $K((y ; \alpha, \delta))$ definida em $(4.1)$ e $\nu$ a valorização de $K(x ; \alpha, \delta)$ definida anteriormente. Então $\left.\omega\right|_{K(x ; \alpha, \delta)}=\nu$ é uma valorização discreta de $K(x ; \alpha, \delta)$. Resumindo, temos: $K(x ; \alpha, \delta)$, um corpo com centro infinito $C_{0},\left[K(x ; \alpha, \delta): C_{0}\right]=\infty$ e $\nu$ uma valorização discreta de $K(x ; \alpha, \delta)$. Além disso, $K((y ; \alpha, \delta))$ é o completamento de $K(x ; \alpha, \delta)$ com a topologia dada por essa valorização, pelo Lema 4.1.2. Logo, pelo Teorema 2.2.4, $K((y ; \alpha, \delta))$ contém um corpo livre $C_{0} \nless X \ngtr$, onde $X$ é um conjunto enumerável.

No caso em que $\delta=0$, defina sobre $K(x ; \alpha)$ a seguinte função: $\eta\left(f g^{-1}\right)=o(f)-o(g)$, onde $o\left(\sum x^{i} a_{i}\right)=\min \left\{i: a_{i} \neq 0\right\}$ é a ordem de $f$, que é uma valorização de $K[x ; \alpha]$. Seja ainda $\eta_{x}$ a valorização de $K((x ; \alpha))$ definida do seguinte modo: se $h=\sum x^{i} a_{i} \in K((x ; \alpha)), a_{i} \in K$, então $\eta_{x}(h)=\sup \left\{n: h \in x^{n} K[[x ; \alpha]]\right\}$. É fácil ver que $\left.\eta_{x}\right|_{K(x ; \alpha)}=\eta$. Temos $\eta(x)=o(x)=1$. Além disso, pode-se mostrar de maneira análoga à demonstração do Lema 4.1 .2 que $K((x ; \alpha))$ é o completamento de $K(x ; \alpha)$ com relação à topologia definida por $\eta$. Temos então o seguinte

Teorema 4.1.5. Seja $K$ um anel com divisão com centro $C$ e seja $\alpha$ um automorfismo de $K$ tal que o corpo $C_{0}=\{c \in C: \alpha(c)=c\}$ seja infinito. Se $K[x ; \alpha]$ for simples ou $\alpha$ tiver ordem interna infinita então o corpo das séries de Laurent $K((x ; \alpha))$ contém um corpo livre $C_{0} \nless X \ngtr$, onde $X$ é um conjunto enumerável.

Demonstração: Sabemos que $Z(K(x ; \alpha))=C_{0}$, que é infinito, por hipótese. Como o conjunto $\left\{x^{i}\right.$ : $i \in \mathbb{Z}\} \subset K(x ; \alpha)$ é linearmente independente sobre $C_{0}$ (pois o é sobre $K$ ) então $\left[K(x ; \alpha): C_{0}\right]=\infty$. Como $\eta$ é uma valorização discreta de $K(x ; \alpha)$ e $K((x ; \alpha))$ é o completamento de $K(x ; \alpha)$ com a 
topologia dada por essa valorização, segue pelo Teorema 2.2.4 que $K((x ; \alpha))$ contém um corpo livre $C_{0} \nless X \ngtr$, onde $X$ é um conjunto enumerável.

Observamos que o teorema acima é uma generalização do Teorema 4 de [3] no qual o corpo $K$ considerado é comutativo.

Vejamos uma aplicação deste último teorema. Seja $k$ um corpo comutativo e seja $\lambda \in K^{*}$. Seja $B_{\lambda}$ a $k$-álgebra gerada por quatro indeterminadas $x, x^{-1}, y, y^{-1}$, sujeita à relação $x y=\lambda y x$, ou mais precisamente, $B_{\lambda}=k\langle x, u, y, v\rangle / I$ onde $I$ é o ideal gerado por $\{x u-1, u x-1, y v-1, v y-1, x y-\lambda y x\}$. Esta álgebra foi definida por Jategaonkar e tratada por Lorenz em [27]. Ela pode ser vista como um quociente $B_{\lambda}=k G /\langle(a, b)-\lambda\rangle$ da álgebra de grupo $k G$, onde $G=\langle a, b:(a,(a, b))=(b,(a, b))=1\rangle$

é o grupo nilpotente livre de índice 2 , ou também como uma extensão de Ore $B_{\lambda}=k\left[x, x^{-1}\right]\left[y, y^{-1} ; \alpha\right]$, onde $\alpha$ é o $k$-automorfismo de $k[x]$ definido por $\alpha(x)=\lambda x$. Segue desta última caracterização que $k[x][y ; \alpha] \subset B_{\lambda} \subset k(x)(y ; \alpha)$ e, portanto, $B_{\lambda}$ é um domínio de Ore com corpo de frações $Q_{\lambda}=$ $k(x)(y ; \alpha)$. Se $\lambda$ não for uma raiz da unidade então $\alpha$ tem ordem infinita e, portanto, pelo Teorema 4.1.3, $Z\left(Q_{\lambda}\right)=\{r \in k(x): \alpha(r)=r\}=k$, que é um corpo infinito. A função ordem $o: k[x][y ; \alpha] \longrightarrow$ $\mathbb{N}, o(y)=1$, é uma valorização e se estende a uma valorização em $B_{\lambda}$ e, conseqüentemente, em $Q_{\lambda}$. Assim, com respeito a essa valorização, pelo Teorema 4.1.5, o completamento $\widehat{Q_{\lambda}}=k(x)((y ; \alpha))$ contém um corpo livre $k \nless X \ngtr$, onde $X$ é um conjunto enumerável. Este exemplo deve ser comparado com o caso considerado no Capítulo 3 , de cujos resultados segue que o corpo clássico de frações de $k G$, para esse mesmo grupo $G$, tem uma valorização que define um completamento contendo um corpo livre.

\subsection{O completamento do corpo de Weyl}

Consideremos agora o corpo de Weyl $K(x)\left(y ; \frac{d}{d x}\right)$. Nesta seção aplicaremos o Teorema 4.1.4 para mostrar que, em característica zero, o completamento do corpo de Weyl, ou seja, o corpo $K(x)\left(\left(z ; \frac{d}{d x}\right)\right), z=y^{-1}$, contém um corpo livre.

Suponhamos que $K$ tenha característica zero e seja $R=K(x)\left[y ; \frac{d}{d x}\right]$. Então $A_{1}(K) \subset R \subset$ $K(x)\left(y ; \frac{d}{d x}\right)$ e $Q(R)=K(x)\left(y ; \frac{d}{d x}\right)$.

Lema 4.2.1 ([18], Corolário 3.16). Seja A um anel simples de característica zero. Então para toda derivação não-interna $\delta$ de $A, A[x ; \delta]$ é um anel simples não-artiniano.

Segue deste último lema que $R$ é um anel simples. Para isso, basta observar que $\frac{d}{d x}$ não é interna. 
De fato, se existisse $u \in K(x)$ tal que $\frac{d}{d x}(v)=u v-v u$, para todo $v \in K(x)$ então, em particular, teríamos $1=\frac{d}{d x}(x)=u x-x u=u x-u x=0$. Temos então o seguinte

Teorema 4.2.2. Seja $K$ um anel com divisão com centro $C$. Se car $(K)=0$ então o completamento do corpo de Weyl, isto é, o corpo das séries de Laurent skew $K(x)\left(\left(z ; \frac{d}{d x}\right)\right)$, contém um corpo livre $C \nless X \ngtr$, onde $X$ é um conjunto enumerável.

Demonstração: Já sabemos que $R$ é simples, pelo lema anterior. Além disso, o corpo $Z:=Z(K(x))_{0}=$ $\left\{v \in Z(K(x)): \frac{d}{d x}(v)=0\right\}$ é infinito pois $C \subset Z(K(x))_{0}$ e $\operatorname{car}(C)=0$. Pelo Teorema 4.1.4, $K(x)\left(\left(z ; \frac{d}{d x}\right)\right)$, contém um corpo livre $Z \Varangle X \ngtr$, onde $X$ é um conjunto enumerável. Como $C \subset Z$ então, pelo Teorema 1.1.15, $K(x)\left(\left(z ; \frac{d}{d x}\right)\right)$ contém o corpo livre $C \nless X \ngtr$.

Vale observar que $C$ é de fato o centro de $Q(R)$. Como $R$ é simples então $Z=Z(Q(R))=$ $Z(K(x))_{0}$, pelo Teorema 4.1.3. Agora, pela Proposição 2.2.3, $Z(K(x))=C(x)$ onde $C$ é o centro de $K$. Logo $Z=C(x)_{0}=\left\{r \in C(x): \frac{d}{d x}(r)=0\right\}$. Seja $r \in C(x)$ um elemento não-nulo tal que $\frac{d}{d x}(r)=0$. Escrevamos $r=f g^{-1}, f, g \in C[x]$, tal que $\operatorname{mdc}(f, g)=1$. Como $0=\frac{d}{d x}(1)=\frac{d}{d x}\left(g g^{-1}\right)=$ $\frac{d}{d x}(g) g^{-1}+g \frac{d}{d x}\left(g^{-1}\right)$ então $g \frac{d}{d x}\left(g^{-1}\right)=-\frac{d}{d x}(g) g^{-1}$ e assim $\frac{d}{d x}\left(g^{-1}\right)=-g^{-1} \frac{d}{d x}(g) g^{-1}$. Logo $0=$ $\frac{d}{d x}\left(f g^{-1}\right)=\frac{d}{d x}(f) g^{-1}-f g^{-1} \frac{d}{d x}(g) g^{-1}$ e, portanto, $\frac{d}{d x}(f)=f g^{-1} \frac{d}{d x}(g)$. Como $C$ é comutativo segue que $\frac{d}{d x}(f) g=f \frac{d}{d x}(g)$ em $C[x]$. Suponha que $\frac{d}{d x}(f) \neq 0$. Como $f$ divide $\frac{d}{d x}(f) g$ e $\operatorname{mdc}(f, g)=1$, então $f$ divide $\frac{d}{d x}(f)$. Mas isso é impossível uma vez que $\operatorname{deg}(f)>\frac{d}{d x}(f)$. Logo $\frac{d}{d x}(f)=0$ e, como $\operatorname{car}(C)=0$, então $f \in C$. Logo $\frac{d}{d x}(g)=0$ o que implica $g \in C$ e, portanto, $r \in C$.

\subsection{O completamento do corpo das matrizes quânticas}

Seja $k$ um corpo comutativo e seja $q \in k$ um elemento não-nulo. A álgebra de matrizes quânticas $2 \times 2$ sobre $k$ é a $k$-álgebra $M_{q}(2)$ dada por geradores $a, b, c, d$ e relações

$$
\begin{aligned}
& a b=q b a \quad a c=q c a \quad b c=c b \\
& b d=q d b \quad c d=q d c \quad a d-d a=\left(q-q^{-1}\right) c b
\end{aligned}
$$

A álgebra $M_{q}(2)$ pode também ser definida como uma extensão de Ore $k[a]\left[b ; \sigma_{b}\right]\left[c ; \sigma_{c}\right]\left[d ; \sigma_{d}, \delta_{d}\right]$, onde $\sigma_{b}: k[a] \longrightarrow k[a], \sigma_{c}: k[a]\left[b ; \sigma_{b}\right] \longrightarrow k[a]\left[b ; \sigma_{b}\right], \sigma_{d}: k[a]\left[b ; \sigma_{b}\right]\left[c ; \sigma_{c}\right] \longrightarrow k[a]\left[b ; \sigma_{b}\right]\left[c ; \sigma_{c}\right]$ são $k$-automorfismos definidos por

$$
\sigma_{b}(a)=q a,
$$




$$
\begin{gathered}
\sigma_{c}(b)=b, \sigma_{c}(a)=q a, \\
\sigma_{d}(c)=q c, \sigma_{d}(b)=q b, \sigma_{d}(a)=a,
\end{gathered}
$$

e $\delta_{d}: k[a]\left[b ; \sigma_{b}\right]\left[c ; \sigma_{c}\right] \longrightarrow k[a]\left[b ; \sigma_{b}\right]\left[c ; \sigma_{c}\right]$ é a $\sigma_{d}$-derivação definida da seguinte maneira: se $f=$ $\sum_{i, j, k \geq 0} c^{i} b^{j} a^{k} \alpha_{i j k} \in k[a]\left[b ; \sigma_{b}\right]\left[c ; \sigma_{c}\right]$, então

$$
\delta_{d}(f)=\sum_{\substack{i, j \geq 0 \\ k>0}} c^{i+1} b^{j+1} a^{k-1}\left(q^{2 k-1}-q^{-1}\right) \alpha_{i j k} .
$$

Esses e outros detalhes sobre matrizes quânticas podem ser encontrados em [17].

Para simplificar as notações vamos escrever $R_{a}=k[a], R_{b}=R_{a}\left[b ; \sigma_{b}\right], R_{c}=R_{b}\left[c ; \sigma_{c}\right]$ e $R_{d}=$ $R_{c}\left[d ; \sigma_{d}, \delta_{d}\right]=M_{q}(2)$. Como $k$ é um corpo então, em particular, $k$ é um domínio noetheriano (à direita e à esquerda). Pela Proposição $1.6 .9, R_{a}=k[a]$ é um domínio noetheriano. Como $\sigma_{b}$ e $\sigma_{c}$ são automorfismos de $R_{a}$ e $R_{b}$, respectivamente, segue pela Proposição 1.6 .9 que $R_{b}=R_{a}\left[b ; \sigma_{b}\right]$ é um domínio noetheriano e, portanto, $R_{c}=R_{b}\left[c ; \sigma_{c}\right]$ também é. Pelo mesmo argumento, $R_{d}$ é um domínio noetheriano. Segue pela Observação 1.6.8 que

$$
Q\left(R_{d}\right)=Q\left(R_{c}\right)\left(d ; \sigma_{d}, \delta_{d}\right)=Q\left(R_{b}\right)\left(c ; \sigma_{c}\right)\left(d ; \sigma_{d}, \delta_{d}\right)=Q\left(R_{a}\right)\left(b ; \sigma_{b}\right)\left(c ; \sigma_{c}\right)\left(d ; \sigma_{d}, \delta_{d}\right)
$$

Seja $R=Q\left(R_{c}\right)\left[d ; \sigma_{d}, \delta_{d}\right]$. Então $R_{d}=R_{c}\left[d ; \sigma_{d}, \delta_{d}\right] \subset R \subset Q\left(R_{d}\right)$ e $Q(R)=Q\left(R_{d}\right)$. Para podermos aplicar o Teorema 4.1 .4 e assim concluirmos que o completamento $\widehat{Q\left(R_{d}\right)}=\widehat{Q(R)}=$ $Q\left(R_{c}\right)\left(\left(e ; \sigma_{d}, \delta_{d}\right)\right), e=d^{-1}$, contém um corpo livre, temos que provar que $R$ é simples ou que $\sigma_{d}$ tem ordem interna infinita. No entanto, observe que $\sigma_{d}$ é um automorfismo interno de $Q\left(R_{c}\right)$. De fato, 
se $f g^{-1} \in Q\left(R_{c}\right), f=\sum c^{i} b^{j} a^{k} \alpha_{i j k}, g=\sum c^{i} b^{j} a^{k} \beta_{i j k} \in R_{c}, g \neq 0$, então

$$
\begin{aligned}
\sigma_{d}\left(f g^{-1}\right) & =\sigma_{d}\left(\sum c^{i} b^{j} a^{k} \alpha_{i j k}\right) \sigma_{d}\left(\sum c^{i} b^{j} a^{k} \beta_{i j k}\right)^{-1} \\
& =\left(\sum c^{i} b^{j} a^{k} q^{i+j} \alpha_{i j k}\right)\left(\sum c^{i} b^{j} a^{k} q^{i+j} \beta_{i j k}\right)^{-1} \\
& =\left(\sum c^{i} b^{j} a^{k} q^{i+j} \alpha_{i j k}\right) a a^{-1}\left(\sum c^{i} b^{j} a^{k} q^{i+j} \beta_{i j k}\right)^{-1} \\
& =\left(\left(\sum c^{i} b^{j} a^{k} q^{i+j} \alpha_{i j k}\right) a\right)\left(\left(\sum c^{i} b^{j} a^{k} q^{i+j} \beta_{i j k}\right) a\right)^{-1} \\
& =\left(\sum c^{i} b^{j} a^{k+1} q^{i+j} \alpha_{i j k}\right)\left(\sum c^{i} b^{j} a^{k+1} q^{i+j} \beta_{i j k}\right)^{-1} \\
& =\left(\sum a c^{i} b^{j} a^{k} \alpha_{i j k}\right)\left(\sum a c^{i} b^{j} a^{k} \beta_{i j k}\right)^{-1} \\
& =a\left(\sum c^{i} b^{j} a^{k} q^{i+j} \alpha_{i j k}\right)\left(\sum c^{i} b^{j} a^{k} \beta_{i j k}\right)^{-1} a^{-1} \\
& =a\left(f g^{-1}\right) a^{-1} .
\end{aligned}
$$

Também não conseguimos provar que $R$ é simples e portanto não podemos aplicar o Teorema 4.1.4. Mesmo assim, mostraremos que $\widehat{Q\left(R_{d}\right)}$ contém um corpo livre se $q$ não for uma raiz da unidade. Para isso, precisamos do seguinte resultado:

Lema 4.3.1 ([28], Teorema pg. 401). Seja $K=k(t)$ o corpo das funções racionais sobre um corpo comutativo $k$ e seja $\alpha$ um k-automorfismo de $K$ de ordem infinita. Então $K(x ; \alpha)$ contém uma k-subálgebra livre não-comutativa.

De agora em diante vamos supor que $q$ não seja uma raiz da unidade. Mostremos que $\sigma_{b}$ é um automorfismo de $k(a)$ de ordem infinita. Suponhamos que $\sigma_{b}$ tenha ordem $n$ para algum $n \geq 1$. Então $\sigma_{b}^{n}=\operatorname{Id}_{k(a)}$. Em particular, $a=\sigma_{b}^{n}(a)=q^{n} a$ e, portanto, $\left(q^{n}-1\right) a=0$ o que implica $q^{n}=1$. Mas isso é uma contradição uma vez que estamos supondo que $q$ não é uma raiz da unidade. Pelo Lema 4.3.1, $Q\left(R_{b}\right)=k(a)\left(b ; \sigma_{b}\right)$ contém uma $k$-subálgebra livre não-comutativa. Como $Q\left(R_{b}\right) \subset Q\left(R_{c}\right) \subset Q(R)$ segue que $Q(R)$ contém uma $k$-subálgebra livre não-comutativa. Logo $Q(R)$ tem dimensão infinita sobre $Z=Z(Q(R))$ (uma vez que qualquer álgebra de dimensão finita $n$ satisfaz o polinômio standard $S_{n+1}$ e, portanto, não pode conter uma álgebra livre). Além disso, como $q$ não é raiz da unidade, $Z$ é infinito. Segue diretamente do Teorema 2.2.4 o seguinte:

Teorema 4.3.2. Nas notaçôes acima, $\widehat{Q\left(R_{d}\right)}=Q\left(R_{c}\right)\left(\left(e ; \sigma_{d}, \delta_{d}\right)\right)$ contém um corpo livre $Z \nless X \ngtr$, sobre um conjunto enumerável $X$. Em particular, $\widehat{Q\left(R_{d}\right)}$ contém $k \nless X \ngtr$, pelo Teorema 1.1.15. 


\section{Capítulo 5}

\section{Uma classe de anéis com valorização $t$-ádica}

Seja $R$ um anel com um elemento central e regular $t$ tal que $\bigcap t^{n} R=0$ e $R / t R$ é um domínio de Ore à direita. Em [25], Lichtman provou que $R$ pode ser imerso num anel com divisão $D$. Mostraremos neste capítulo que, em alguns casos, tal anel com divisão $D$ contém um corpo livre. Também neste capítulo provaremos a existência de um corpo livre no completamento de um corpo de frações da envolvente universal de uma álgebra de Lie em característica zero.

\subsection{O completamento de um anel com divisão gerado por um anel com valoriza- ção $t$-ádica}

Seja $R$ um anel e seja $t$ um elemento central e regular de $R$ tal que $\bigcap t^{n} R=0$ e $R / t R$ é um domínio. Como vimos no Exemplo 1.3.3(1), $R$ tem uma valorização $\nu_{t}$ definida por $\nu_{t}(x)=\sup \left\{n: x \in t^{n} R\right\}$, chamada valorização $t$-ádica . Com algumas hipóteses adicionais é possível mostrar que $R$ pode ser imerso num anel com divisão. Mais precisamente Lichtman provou o seguinte

Teorema 5.1.1 ([25], Teorema 1). Seja $R$ um anel e $t$ um elemento central e regular de $R$. Assuma $q u e \bigcap t^{n} R=0$ e que $R / t R$ é um dominio de Ore à direita. Então $R$ pode ser imerso em um anel com divisão $D$, a valorização t-ádica $\nu_{t}$ se estende a uma valorização $\nu$ de $D$ e o subconjunto $R\left(R^{*}\right)^{-1}$ é denso em $D$. Além disso, se $S$ é o anel de valorização de $D$ então $S / t S$ é isomorfo a $\Delta$, o corpo de frações de $R / t R$.

Este teorema também pode ser visto em [8], Teorema 2.6.2. Também segue da demonstração desse teorema que $t$ é um elemento central de $D, S$ é um anel local com ideal maximal $t S$ e, além disso, $t S$ define uma valorização $t$-ádica em $S$, que estende $\nu_{t}$ e que é igual a $\left.\nu\right|_{S}$. 
Fixemos uma constante real $c \in(0,1)$ e considere a métrica definida sobre $D$ por $d(x, y)=c^{\nu(x-y)}$. Não é difícil provar que $D$ é completo com a topologia definida por essa métrica. Provemos agora o seguinte teorema, o qual fornece uma condição suficiente para garantir a existência de um corpo livre no anel com divisão $D$. Este é mais uma aplicação do Teorema 2.2.4.

Teorema 5.1.2. Nas condições do teorema acima, se $\Delta$ tem dimensão infinita sobre $Z(\Delta)$, então $D$ contém um corpo livre $Z(D) \nless X \ngtr$, onde $X$ é um conjunto enumerável.

Demonstração: Uma vez que $D$ é uma localização de $S$ (mais precisamente, $D=S T^{-1}$, onde $T=$ $\left.\left\{1, t, t^{2}, \ldots\right\}\right)$ então $\nu$ é uma valorização discreta de $D$. Como as potências de $t$ são todas distintas (pois, caso contrário $t$ seria inversível em $R$ ) então $Z(D)$ é infinito. Mostremos agora que $D$ tem dimensão infinita sobre $Z(D)$. Suponhamos que $[D: Z(D)]=n<\infty$. Sejam $s_{1}+t S, s_{2}+$ $t S, \ldots, s_{n+1}+t S, n+1$ elementos distintos de $S / t S$. Então $s_{1}, s_{2}, \ldots, s_{n+1}$, são $n+1$ elementos distintos de $D$. Existem $z_{1}, z_{2}, \ldots, z_{n+1} \in Z(D)$, não todos nulos, tais que $\sum_{i=1}^{n+1} z_{i} s_{i}=0$. Podemos então escrever $\sum_{j=1}^{m} z_{i_{j}} s_{i_{j}}=0$, onde $i_{j} \in\{1, \ldots, n+1\}, j=1, \ldots, m$, são tais que $z_{i_{j}} \neq 0$. Se $\nu\left(z_{i_{j}}\right)=$ $l_{j}$ então $\nu\left(z_{i_{j}} t^{-l_{j}}\right)=\nu\left(z_{i_{j}}\right)-l_{j} \nu(t)=\nu\left(z_{i_{j}}\right)-l_{j}=0$. Logo $u_{j}:=z_{i_{j}} t^{-l_{j}} \in S \backslash t S$. Assim, $z_{i_{j}}=u_{j} t^{l_{j}}$, com $u_{j} \in S \backslash t S$. Como $z_{i_{j}} \in Z(D)$ então, para todo $d \in D, u_{j} t^{l_{j}} d=d u_{j} t^{l_{j}}$ e, portanto, $u_{j} d=d u_{j}$. Logo $u_{j} \in Z(D) \cap S \subset Z(S)$, para todo $j=1, \ldots, m$. Temos $0=\sum_{j=1}^{m} u_{j} t^{l_{j}} s_{i_{j}}=\sum_{j=1}^{m} u_{j} s_{i_{j}} t^{l_{j}}$. Seja $r=\min \left\{l_{j}: j=1, \ldots, m\right\}$. Sem perda de generalidade podemos supor que $r=l_{1}$. Então $l_{j}-r \geq 0$, e portanto $t^{l_{j}-r} \in R \subset S$, para todo $1 \leq j \leq m$. Assim $\left(\sum_{j=1}^{m} u_{j} s_{i_{j}} t^{l_{j}-r}\right) t^{r}=0$ o que implica $0=\sum_{j=1}^{m} u_{j} s_{i_{j}} t^{l_{j}-r}=u_{1} s_{i_{1}}+\sum_{j=2}^{m} u_{j} s_{i_{j}} t^{l_{j}-r}$. Em $S / t S$ temos $0=\left(u_{1}+t S\right)\left(s_{i_{1}}+t S\right)+\sum_{j=2}^{m}\left(u_{j} t^{l_{j}-r}+\right.$ $t S)\left(s_{i_{j}}+t S\right)$, onde $u_{1}+t S, u_{j} t^{l_{j}-r}+t S \in Z(S / t S)$, para todo $j=1, \ldots, m$, com $u_{1}+t S \neq 0$. Logo $\left\{s_{i_{j}}+t S: j=1, \ldots, m\right\} \subset S / t S$ é linearmente dependente sobre $Z(S / t S)$ e, portanto, o conjunto $\left\{s_{i}+t S: i=1, \ldots, n+1\right\}$ também é. Portanto $[\Delta: Z(\Delta)]=[S / t S: Z(S / t S)]<n+1$, o que é uma contradição. Portanto $D$ tem dimensão infinita sobre $Z(D)$. Como $D$ é completo segue pelo Teorema 2.2.4 que $D$ contém um corpo livre $Z(D) \nless X \ngtr$, onde $X$ é um conjunto enumerável.

\subsection{O completamento do corpo de Lie}

Seja $L$ uma álgebra de Lie sobre um corpo comutativo $k$. Como dissemos na Seção 1.7, Cohn provou que a envolvente universal $U(L)$ pode ser imersa num corpo $D(L)$, chamado corpo de Lie; mais ainda, $D(L)$ está contido num corpo maior que será denotado por $\widehat{D(L)}$. Nosso objetivo nesta seção será provar que, em característica zero, $\widehat{D(L)}$ contém um corpo livre. Para isso precisamos de algumas definições e resultados. 
Um anel filtrado $R$ é um anel com uma série de $\mathbb{Z}$-submódulos indexados por $\mathbb{Z}$,

$$
\cdots \supset R_{-1} \supset R_{0} \supset R_{1} \supset \cdots,
$$

tais que

(1) $R_{i} R_{j} \subset R_{i+j}$,

(2) $\cap R_{n}=0$,

(3) $\bigcup R_{n}=R$.

Se em (5.1) $R_{0}=R$ e $R_{-1}, R_{-2}, \ldots$ estão ausentes dizemos que a filtração é positiva. Analogamente, quando $R_{1}=R_{2}=\cdots=0$, temos uma filtração negativa (neste caso, é conveniente trocar o sinal dos índices).

Sobre um anel filtrado podemos definir uma função com valores em $\mathbb{Z}$ colocando

$$
\nu(x)=\sup \left\{n: x \in R_{n}\right\} .
$$

É fácil verificar que $\nu$ satisfaz

(1) $\nu(x) \in \mathbb{Z}$ se $x \neq 0, \nu(0)=\infty$,

(2) $\nu(x+y) \geq \min \{\nu(x), \nu(y)\}$,

(3) $\nu(x y) \geq \nu(x)+\nu(y)$.

Tal função é chamada subvalorização, pseudovalorização ou também filtração de $R$. Obviamente, se vale a igualdade em (3), $\nu$ é uma valorização.

Quando a filtração é negativa e mudamos o sinal dos índices em (5.1), é fácil ver que $\nu$ passa a ser dada por

$$
\nu(x)=-\inf \left\{n: x \in R_{n}\right\} .
$$

Um anel $R$ é dito ser graduado se ele é uma soma direta de $\mathbb{Z}$-submódulos indexados por $\mathbb{Z}$ : $R=\bigoplus A_{n}$ tais que $A_{i} A_{j}=A_{i+j}$. Um elemento de $A_{i}$ é dito ser homogêneo de grau $i$. Todo anel graduado pode ser filtrado definindo $R_{n}=\sum_{i=n}^{\infty} A_{i}$. A todo anel filtrado podemos associar um anel 
graduado $G(R)$, cujo grupo aditivo é a soma direta $\bigoplus\left(R_{n} / R_{n+1}\right)$, com multiplicação definida como segue: dados $\alpha \in R_{i} / R_{i+1}, \beta \in R_{j} / R_{j+1}$, tomamos representantes $a \in R_{i}$ para $\alpha$ e $b \in R_{j}$ para $\beta$ e colocamos

$$
\alpha \beta=\left(a+R_{i+1}\right)\left(b+R_{j+1}\right)=a b+R_{i+j+1} .
$$

Proposição 5.2.1 ([8], Proposição 2.6.1). Seja $R$ um anel filtrado com filtração associada $\nu$ e anel graduado $G(R)$. Então $G(R)$ é um domínio se e somente se $\nu$ for uma valorização. Neste caso, o próprio anel $R$ é um domínio.

O teorema seguinte foi provado por Cohn em [4] e posteriormente foi redemonstrado por Lichtman usando o Teorema 5.1.1.

Teorema 5.2.2 ([8], Teorema 2.6.5). Seja $R$ um anel filtrado cujo anel graduado associado é um domínio de Ore à direita. Então $R$ pode ser imerso em um corpo $D$, a valorização induzida da filtração de $R$ se estende a $D$ e $D$ é completo na topologia definida por essa valorização.

Se $L$ é uma álgebra de Lie sobre um corpo comutativo $k, L$ gera $U(L)$ como álgebra. Logo $U(L)$ tem uma filtração canônica dada pelos subespaços

$$
U^{-1}=0, \quad U^{i}=k+L+L^{2}+\cdots+L^{i}, i \geq 0
$$

onde $L^{i}$ é o subespaço gerado por todos os produtos de $i$ elementos de $L$. Segue do Teorema de Poincaré-Birkhoff-Witt que o anel graduado associado a essa filtração é um domínio comutativo (ver [8, pg. 89]) e portanto a filtração associada $\nu: U(L) \longrightarrow \mathbb{Z} \cup\{\infty\}$ dada por $\nu(x)=-\inf \left\{n: x \in U^{n}\right\}$ é uma valorização. Em particular, $U(L)$ é um domínio. Vamos chamar $\nu$ de valorização canônica de $U(L)$. Uma conseqüência particularmente interessante do Teorema 5.2.2 é o seguinte

Teorema 5.2.3 ([8], Teorema 2.6.6). A envolvente universal associativa de qualquer álgebra de Lie sobre um corpo comutativo pode ser imersa em um anel com divisão $\widehat{D(L)}$, o qual é completo na topologia definida pela valorização canônica de $U(L)$.

O anel com divisão $D(L)$ citado acima é o subcorpo de $\widehat{D(L)}$ gerado por $U(L)$. Como $\widehat{D(L)}$ é completo então o fecho $\overline{D(L)}$ do subespaço $D(L)$ do espaço métrico $\widehat{D(L)}$ é completo e, portanto, $\overline{D(L)}$ é o completamento de $D(L)$. 
Lichtman provou em [26] que, se $\operatorname{car}(k)=0$, então qualquer anel com divisão que contenha $U(L)$, contém uma $k$-álgebra livre de posto $2 \mathrm{e}$, portanto, tem dimensão infinita sobre seu centro, que é infinito, pois $\operatorname{car}(k)=0$. Segue imediatamente do Teorema 2.2.4 e do Teorema 5.2.3 o seguinte

Teorema 5.2.4. Seja L uma álgebra de Lie não-abeliana sobre um corpo comutativo de característica zero e seja $U(L)$ sua envolvente universal associativa. Seja $\widehat{D(L)}$ o corpo obtido no Teorema 5.2.3 e $D(L)$ o subcorpo de $\widehat{D(L)}$ gerado por $U(L)$. Então $\overline{D(L)}$ contém um corpo livre $Z(D(L)) \nless X \ngtr$, sobre um conjunto enumerável $X$ e, portanto, $\widehat{D(L)}$ contém $Z(D(L)) \nless X \ngtr$. 


\section{Capítulo 6}

\section{Alguns anéis com divisão que não contêm corpos livres}

Seja $K$ um corpo comutativo e seja $G$ um grupo nilpotente, finitamente gerado, livre de torção, não-abeliano. Vimos na Seção 3.2 que o completamento do corpo clássico de frações $Q(K G)$, que está contido em $K((G))$, contém um corpo livre $Z \nless X$ ஓ, sobre um conjunto enumerável $X$, onde $Z$ é o centro de $Q(K G)$. Como $K \subset Z$ segue pelo Teorema 1.1.15 que $K((G))$ contém o corpo livre $K \nless X \ngtr$. Já na Seção 4.2 mostramos que o completamento do corpo de Weyl $K(x)\left(y ; \frac{d}{d x}\right)$ contém um corpo livre sobre $K$, se $\operatorname{car}(K)=0$. Neste momento, é natural surgirem as seguintes perguntas (principalmente se o leitor conhece os artigos [29] e [30] das referências): O corpo de Weyl contém um corpo livre sobre $K$ ? E quanto ao corpo de frações da álgebra de grupo acima? A resposta para essas perguntas é não, como veremos neste capítulo.

\subsection{Um resultado geral}

Recordemos o seguinte conceito envolvendo produtos tensoriais de módulos:

Definição 6.1.1. Seja $R$ um anel e seja $M$ um $R$-módulo à direita. Dizemos que $M$ é fielmente plano se dada uma seqüência de $R$-módulos à esquerda

$$
N^{\prime} \stackrel{f}{\rightarrow} N \stackrel{g}{\rightarrow} N^{\prime \prime}
$$

a seqüência

$$
M \otimes_{R} N^{\prime} \stackrel{1_{M} \otimes f}{\longrightarrow} M \otimes_{R} N \stackrel{1_{M} \otimes g}{\longrightarrow} M \otimes_{R} N^{\prime \prime}
$$

é exata se e somente se a seqüência (6.1) for exata. 
Módulos à esquerda fielmente planos são definidos de maneira análoga.

Proposição 6.1.2. Todo módulo livre é fielmente plano.

Demonstração: Ver Exemplo 2 pg. 29 de [1].

Proposição 6.1.3 ([1], Proposição 2 pg. 29). Seja $M$ um R-módulo à direita fielmente plano e seja $f: N^{\prime} \longrightarrow N$ um homomorfismo de $R$-módulos à esquerda. Então $f$ é injetor (respectivamente sobrejetor, bijetor) se e somente se $1_{M} \otimes f: M \otimes_{R} N^{\prime} \longrightarrow M \otimes_{R} N$ for.

Seja $R$ uma $k$-álgebra, $k$ um corpo comutativo. Seja $R^{o p}$ o anel oposto a $R$, ou seja, $R^{o p}=\left\{r^{o p}\right.$ : $r \in R\}$, com adição e multiplicação definidas por

$$
a^{o p}+b^{o p}=(a+b)^{o p} \quad \text { e } \quad a^{o p} b^{o p}=(b a)^{o p} .
$$

Como $k$ é central em $R$ então $R^{o p}$ também é uma $k$-álgebra. Considere o produto tensorial $R \otimes_{k} R^{o p}$, que é uma $k$-álgebra, onde a multiplicação é dada por

$$
\left(a_{1} \otimes b_{1}^{o p}\right)\left(a_{2} \otimes b_{2}^{o p}\right)=a_{1} a_{2} \otimes b_{1}^{o p} b_{2}^{o p}=a_{1} a_{2} \otimes\left(b_{2} b_{1}\right)^{o p} .
$$

$\mathrm{O}$ anel $R$ pode ser visto como um $R \otimes_{k} R^{o p}$-módulo à esquerda se definimos

$$
\left(\sum a_{i} \otimes b_{i}^{o p}\right) r=\sum a_{i} r b_{i}, a_{i}, b_{i}, r \in R
$$

Seja

$$
\begin{aligned}
\mu: R \otimes_{k} R^{o p} & \longrightarrow R \\
a \otimes b^{o p} & \longmapsto a b
\end{aligned} .
$$

Então $\mu$ é um homomorfismo sobrejetor de $R \otimes_{k} R^{o p}$-módulos à esquerda. O conjunto $\left\{\sum a_{i} \otimes b_{i}^{o p}\right.$ : $\left.\sum a_{i} b_{i}=0\right\}$ é o núcleo desse homomorfismo e é portanto um ideal à esquerda de $R \otimes_{k} R^{o p}$.

Lema 6.1.4. Nas notações acima, $\operatorname{Ker}(\mu)$ é gerado como $R$-módulo à esquerda pelos elementos $x \otimes 1^{o p}-1 \otimes x^{o p}, x \in R$.

Demonstração: Ver Proposição 2.2.5 de [7].

O lema abaixo nos foi apresentado por Lichtman. 
Lema 6.1.5. Seja $D$ um anel com divisão o qual é uma $k$-álgebra sobre um corpo comutativo $k$. Suponha que D contenha uma cadeia infinita de $k$-subálgebras com divisão

$$
D_{1} \varsubsetneqq D_{2} \varsubsetneqq D_{3} \varsubsetneqq \cdots
$$

Denotemos por $R_{n}:=D_{n} \otimes_{k} D_{n}^{o p}, \mu_{n}: R_{n} \longrightarrow D_{n}, \mu_{n}\left(a \otimes b^{o p}\right)=a b$, e $I_{n}:=\operatorname{Ker}\left(\mu_{n}\right)$. Então a álgebra $R:=D \otimes_{k} D^{o p}$ contém uma cadeia infinita de ideais à esquerda

$$
R I_{1} \varsubsetneqq R I_{2} \varsubsetneqq R I_{3} \varsubsetneqq \cdots
$$

Demonstração: Como $D_{n} \varsubsetneqq D_{n+1}$ então $R_{n} \varsubsetneqq R_{n+1}$. Se $\sum a_{i} \otimes b_{i}^{o p} \in I_{n}$ então $\sum a_{i} \otimes b_{i}^{o p} \in R_{n+1} \mathrm{e}$ $\sum a_{i} b_{i}=0$. Logo $\sum a_{i} \otimes b_{i}^{o p} \in I_{n+1}$ e, portanto, $I_{n} \subset I_{n+1}$ o que implica $R I_{n} \subset R I_{n+1}$, para todo $n \geq 1$. Queremos mostrar agora que essa inclusão é estrita. Por analogia é suficiente mostrar que $R I_{1} \varsubsetneqq R I_{2}$.

Como $I_{1} \subset I_{2}$ e $I_{2}$ é um ideal à esquerda de $R_{2}$ então $R_{2} I_{1} \subset I_{2}$. Suponhamos que $R_{2} I_{1}=I_{2}$. Como $\mu_{2}$ é um homomorfismo sobrejetor de $R_{2}$-módulos à esquerda então $D_{2} \cong R_{2} / I_{2}=R_{2} / R_{2} I_{1}$. Como $D_{1} \subset D_{2}$ então $D_{1}^{o p} \subset D_{2}^{o p}$. Logo $D_{2}^{o p}$ é um $D_{1}^{o p}$-módulo à direita com multiplicação por escalar dada pela multiplicação de $D_{2}^{o p}$; portanto, $D_{2}^{o p}$ é um $\left(D_{1}^{o p}\right)^{o p}=D_{1}$-módulo à esquerda com multiplicação por escalar dada por $d_{1} d_{2}^{o p}=\left(d_{1}^{o p}\right)^{o p} d_{2}^{o p}=d_{2}^{o p} d_{1}^{o p}=\left(d_{1} d_{2}\right)^{o p}$. Consideremos então $S=D_{2} \otimes_{D_{1}} D_{2}^{o p}$. Seja $x \in D_{2} \backslash D_{1}$. Então $\left\{1^{o p}, x^{o p}\right\} \subset D_{2}^{o p}$ é linearmente independente sobre $D_{1}$ à esquerda. Completemos $\left\{1^{o p}, x^{o p}\right\}$ a uma base de $D_{2}^{o p}$ sobre $D_{1}:\left\{1^{o p}, x^{o p}, x_{j}^{o p}: j \in J\right\}$. Segue pelo Corolário 1.7.16 de [40] que $S$ é um $D_{2}$-módulo livre à esquerda com base $\left\{1 \otimes_{D_{1}} 1^{o p}, 1 \otimes_{D_{1}}\right.$ $\left.x^{o p}, 1 \otimes_{D_{1}} x_{j}^{o p}: j \in J\right\}$. Em particular, $\left\{1 \otimes_{D_{1}} 1^{o p}, 1 \otimes_{D_{1}} x^{o p}\right\}$ é linearmente independente sobre $D_{2}$ à esquerda.

É fácil ver que existe um único homomorfismo de grupos aditivos $\varphi: R_{2} \longrightarrow S$ tal que $\varphi\left(x \otimes_{k}\right.$ $\left.y^{o p}\right)=x \otimes_{D_{1}} y^{o p}$. Observe agora que $S$ é um $R_{2}$-módulo à esquerda se definimos $\left(a \otimes_{k} b^{o p}\right)\left(x \otimes_{D_{1}} y^{o p}\right)=$ $a x \otimes_{D_{1}} b^{o p} y^{o p}=a x \otimes_{D_{1}}(y b)^{o p}$, para todos $a, b, x, y \in D_{2}$. Como

$$
\begin{aligned}
\varphi\left(\left(a \otimes_{k} b^{o p}\right)\left(x \otimes_{k} y^{o p}\right)\right) & =\varphi\left(a x \otimes_{k}(y b)^{o p}\right)=a x \otimes_{D_{1}}(y b)^{o p} \\
& =\left(a \otimes_{k} b^{o p}\right)\left(x \otimes_{D_{1}} y^{o p}\right)=\left(a \otimes_{k} b\right) \varphi\left(x \otimes_{k} y^{o p}\right)
\end{aligned}
$$

então $\varphi$ é um homomorfismo de $R_{2}$-módulos à esquerda. Claramente, $\varphi$ é sobrejetor. Além disso, se 
$a, b \in D_{1}$ e $x, y \in D_{2}$, então

$$
\begin{aligned}
\varphi\left(\left(x \otimes_{k} y^{o p}\right) b\left(a \otimes_{k} 1^{o p}-1 \otimes_{k} a^{o p}\right)\right) & =\varphi\left(\left(x \otimes_{k} y^{o p}\right)\left(b a \otimes_{k} 1^{o p}-b \otimes_{k} a^{o p}\right)\right. \\
& =\varphi\left(x b a \otimes_{k} y^{o p}-x b \otimes_{k}(a y)^{o p}\right) \\
& =x b a \otimes_{D_{1}} y^{o p}-x b \otimes_{D_{1}}(a y)^{o p} \\
& =x b \otimes_{D_{1}} a y^{o p}-x b \otimes_{D_{1}}(a y)^{o p} \\
& =x b \otimes_{D_{1}}(a y)^{o p}-x b \otimes_{D_{1}}(a y)^{o p}=0 .
\end{aligned}
$$

Segue pelo Lema 6.1.4 que $R_{2} I_{1} \subset \operatorname{Ker}(\varphi)$. Pelo Teorema do isomorfismo existe um único homomorfismo (sobrejetor) de $R_{2}$-módulos à esquerda $\bar{\varphi}: R_{2} / R_{2} I_{1} \longrightarrow S$ tal que o diagrama abaixo comuta.

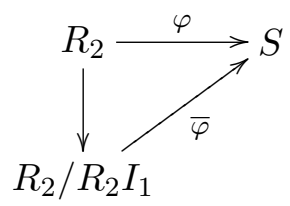

Como $\mu_{2}: R_{2} \longrightarrow D_{2}$ é um homomorfismo sobrejetor de $R_{2}$-módulos à esquerda e $R_{2} I_{1}=I_{2}=$ $\operatorname{Ker}\left(\mu_{2}\right)$ então existe um único isomorfismo $\overline{\mu_{2}}: R_{2} / R_{2} I_{1} \longrightarrow D_{2}$ tal que o diagrama abaixo comuta.

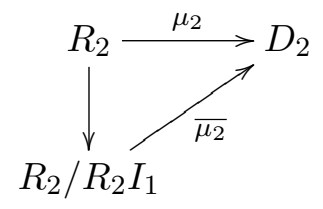

Seja $\psi$ a seguinte composta

$$
D_{2} \stackrel{{\overline{\mu_{2}}}^{-1}}{\longrightarrow} R_{2} / R_{2} I_{1} \stackrel{\bar{\varphi}}{\rightarrow} S
$$

Tome um elemento $x \in D_{2} \backslash D_{1}$. Temos

$$
\psi(x)=\bar{\varphi}{\overline{\mu_{2}}}^{-1}(x)=\bar{\varphi}\left(x \otimes_{k} 1^{o p}+R_{2} I_{1}\right)=\varphi\left(x \otimes_{k} 1^{o p}\right)=x \otimes_{D_{1}} 1^{o p}
$$

e também

$$
\psi(x)=\bar{\varphi}{\overline{\mu_{2}}}^{-1}(x)=\bar{\varphi}\left(1 \otimes_{k} x^{o p}+R_{2} I_{1}\right)=\varphi\left(1 \otimes_{k} x^{o p}\right)=1 \otimes_{D_{1}} x^{o p}
$$


e, portanto, $x \otimes_{D_{1}} 1^{o p}=1 \otimes_{D_{1}} x^{o p}$ de onde segue que $x\left(1 \otimes_{D_{1}} 1^{o p}\right)-1\left(1 \otimes_{D_{1}} x^{o p}\right)=0$. Mas isso é um absurdo pois sabemos que $\left\{1 \otimes_{D_{1}} 1^{o p}, 1 \otimes_{D_{1}} x^{o p}\right\}$ é linearmente independente sobre $D_{2}$ à esquerda. Portanto, $R_{2} I_{1} \varsubsetneqq I_{2}$.

Suponhamos que $R I_{1}=R I_{2}$. Seja $f: R_{2} \otimes_{R_{1}} I_{1} \longrightarrow R_{2} I_{1}$ o único homomorfismo de grupos aditivos tal que $f\left(x \otimes_{R_{1}} a\right)=x a$. Como

$$
f\left(y\left(x \otimes_{R_{1}} a\right)\right)=f\left(y x \otimes_{R_{1}} a\right)=(y x) a=y(x a)=y f\left(x \otimes_{R_{1}} a\right),
$$

para quaisquer $x, y \in R_{2}$ e $a \in I_{1}$, então $f$ é um homomorfismo de $R_{2}$-módulos à esquerda. Além disso, se $x a \in R_{2} I_{1}$, então $x a=f\left(x \otimes_{R_{1}} a\right)$ e portanto $f$ é sobrejetor. Seja $\sum_{i} x_{i} \otimes_{R_{1}} a_{i} \in \operatorname{Ker}(f)$. Como $D_{2}$ é um $D_{1}$-módulo à direita livre e $D_{2}^{o p}$ é um $D_{1}^{o p}$-módulo à direita livre então $R_{2}=D_{2} \otimes_{k} D_{2}^{o p}$ é um $R_{1}=D_{1} \otimes_{k} D_{1}^{o p}$-módulo à direita livre, pelo Lema pg. 166 e pelo Exercício 2 pg. 168 de [36]. Seja $\left\{v_{l}: l \in L\right\}$ uma base de $R_{2}$ sobre $R_{1}$ à direita. Então $x_{i}=\sum_{l} v_{l} \alpha_{i l}$, para alguns $\alpha_{i l} \in R_{1}$. Assim,

$$
\begin{aligned}
\sum_{i} x_{i} \otimes_{R_{1}} a_{i} & =\sum_{i}\left(\sum_{l} v_{l} \alpha_{i l}\right) \otimes_{R_{1}} a_{i}=\sum_{i, l} v_{l} \alpha_{i l} \otimes_{R_{1}} a_{i}=\sum_{i, l} v_{l} \otimes_{R_{1}}\left(\alpha_{i l} a_{i}\right) \\
& =\sum_{l} v_{l} \otimes_{R_{1}}\left(\sum_{i} \alpha_{i l} a_{i}\right)
\end{aligned}
$$

e, portanto,

$$
\left.0=f\left(\sum_{i} x_{i} \otimes_{R_{1}} a_{i}\right)=f\left(\sum_{l} v_{l} \otimes_{R_{1}}\left(\sum_{i} \alpha_{i l} a_{i}\right)\right)\right)=\sum_{l} v_{l}\left(\sum_{i} \alpha_{i l} a_{i}\right) .
$$

Como $\sum_{i} \alpha_{i l} a_{i} \in I_{1} \subset R_{1}$ devemos ter $\sum_{i} \alpha_{i l} a_{i}=0$, para todo $l$, de onde segue que $\sum_{i} x_{i} \otimes_{R_{1}} a_{i}=$ $\sum_{l} v_{l} \otimes_{R_{1}}\left(\sum_{i} \alpha_{i l} a_{i}\right)=0$. Portanto $f$ é injetor. Logo $R_{2} \otimes_{R_{1}} I_{1} \cong R_{2} I_{1}$, como $R_{2}$-módulos à esquerda.

De modo análogo ao que foi feito acima, também podemos mostrar que existe um isomorfismo de $R$-módulos à esquerda

$$
\begin{aligned}
g_{n}: R \otimes_{R_{n}} I_{n} & \longrightarrow R I_{n} \\
x \otimes_{R_{n}} a & \longmapsto x a
\end{aligned}
$$


para todo $n \geq 1$. Além disso, é fácil mostrar que existe um isomorfismo de $\left(R, R_{n}\right)$-bimódulos

$$
\begin{aligned}
h_{n}: R \otimes_{R_{n}} R_{n} & \longrightarrow R \\
r \otimes_{R_{n}} x & \longmapsto r x
\end{aligned},
$$

para todo $n \geq 1$. Seja $\theta$ a seguinte composta

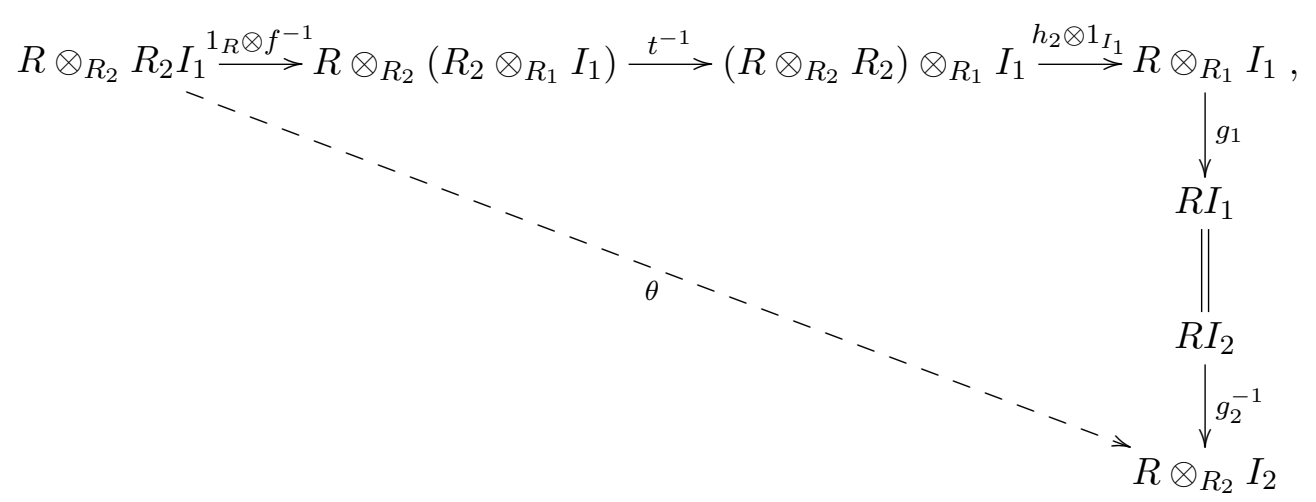

onde $t:\left(R \otimes_{R_{2}} R_{2}\right) \otimes_{R_{1}} I_{1} \longrightarrow R \otimes_{R_{2}}\left(R_{2} \otimes_{R_{1}} I_{1}\right)$ é o isomorfismo de $R$-módulos à esquerda dado por $\left(x_{1} \otimes x_{2}\right) \otimes x_{3} \mapsto x_{1} \otimes\left(x_{2} \otimes x_{3}\right)$. Logo $\theta$ é um isomorfismo de $R$-módulos à esquerda. Se $r \otimes_{R_{2}} r_{2} a \in R \otimes_{R_{2}} R_{2} I_{1}$, então

$$
\begin{aligned}
\theta\left(r \otimes_{R_{2}} r_{2} a\right) & =g_{2}^{-1} g_{1}\left(h_{2} \otimes 1_{I_{1}}\right) t^{-1}\left(1_{R} \otimes f^{-1}\right)\left(r \otimes_{R_{2}} r_{2} a\right) \\
& =g_{2}^{-1} g_{1}\left(h_{2} \otimes 1_{I_{1}}\right) t^{-1}\left(r \otimes_{R_{2}} f^{-1}\left(r_{2} a\right)\right) \\
& =g_{2}^{-1} g_{1}\left(h_{2} \otimes 1_{I_{1}}\right) t^{-1}\left(r \otimes_{R_{2}}\left(r_{2} \otimes_{R_{1}} a\right)\right) \\
& =g_{2}^{-1} g_{1}\left(h_{2} \otimes 1_{I_{1}}\right)\left(\left(r \otimes_{R_{2}} r_{2}\right) \otimes_{R_{1}} a\right) \\
& =g_{2}^{-1} g_{1}\left(h_{2}\left(r \otimes_{R_{2}} r_{2}\right) \otimes_{R_{1}} a\right) \\
& =g_{2}^{-1} g_{1}\left(\left(r r_{2} \otimes_{R_{1}} a\right)\right. \\
& =g_{2}^{-1}\left(\left(r r_{2}\right) a\right)=g_{2}^{-1}\left(r\left(r_{2} a\right)\right) \\
& =r \otimes_{R_{2}} r_{2} a .
\end{aligned}
$$

Logo $\theta=1_{R} \otimes \iota$, onde $\iota: R_{2} I_{1} \longrightarrow I_{2}$ é a inclusão natural que, obviamente, é um homomorfismo de $R_{2}$-módulos à esquerda. Pelo Lema pg. 166 e pelo Exercício 2 pg. 168 de [36], $R$ é um $R_{2}$-módulo à direita livre. Logo, pela Proposição 6.1.2, $R$ é um $R_{2}$-módulo à direita fielmente plano. Como 
$\theta=1_{R} \otimes \iota$ é um isomorfismo segue pela Proposição 6.1 .3 que $\iota$ é um isomorfismo. Mas isso é um absurdo uma vez que nós mostramos que $R_{2} I_{1} \varsubsetneqq I_{2}$. Portanto, $R I_{1} \varsubsetneqq R I_{2}$, como queríamos demonstrar.

Lema 6.1.6 ([8], Corolário 5.5.9). Seja D um anel com divisão e seja $K$ um subcorpo de D. Então todo corpo livre da forma $D_{K} \nless x_{1}, x_{2}, \ldots \ngtr$ pode ser imerso em $D_{K} \nless x, y \ngtr$, mapeando $x_{r} \mapsto y^{-r} x y^{r}$, $r=1,2, \ldots$

Corolário 6.1.7. Todo corpo livre não-comutativo contém um corpo livre com um sistema infinito de geradores.

Demonstração: Seja $D$ um corpo com subcorpo $K$ e seja $X$ um conjunto não-vazio. Seja $Y$ um subconjunto não-vazio de $X$ tal que $Y \varsubsetneqq X$. Escreva $Z:=X \backslash Y$. Pelo Lema 5.4.2 de [8], o homomorfismo natural de $D$-anéis $f: D_{K}\langle Y\rangle \longrightarrow D_{K}\langle X\rangle=D_{K}\langle Y\rangle *_{K} K\langle Z\rangle$ é honesto. Logo $f$ se estende a um monomorfismo $D_{K} \nless Y \ngtr \longrightarrow D_{K} \nless X \ngtr$, pela Observação 1.1.17.

Seja $D_{K} \nless x_{1}, x_{2}, \ldots, x_{n} \ngtr$ um corpo livre com $n \geq 2$. Pelo que acabamos de mostrar, temos a inclusão $D_{K} \nless x_{1}, x_{2} \ngtr \subset D_{K} \nless x_{1}, x_{2}, \ldots, x_{n} \ngtr$. Pelo corolário anterior, $D_{K} \nless x_{1}, x_{2} \ngtr$ contém um corpo livre com um sistema enumerável de geradores e, portanto, $D_{K} \nless x_{1}, x_{2}, \ldots, x_{n} \ngtr$ também contém.

O seguinte lema também nos foi apresentado por Lichtman e fornece uma condição suficiente para que um anel com divisão não contenha um corpo livre.

Lema 6.1.8. Seja $D$ uma k-álgebra com divisão sobre um corpo comutativo $k$. Se $D$ contém um corpo livre sobre $k$ então $D \otimes_{k} D^{o p}$ não pode ser um anel noetheriano à esquerda.

Demonstração: Se $D$ contém um corpo livre sobre $k$ então $D$ contém um corpo livre com um número infinito enumerável de geradores, digamos, $k_{C} \nless x_{1}, x_{2}, \ldots \ngtr$, onde $C$ é um subcorpo de $k$. Como $k_{C} \nless x_{1}, x_{2}, \ldots, x_{n} \ngtr \subset k_{C} \nless x_{1}, x_{2}, \ldots, x_{n+1} \ngtr$, pela demonstração do corolário anterior, então, tomando $D_{n}=k_{C} \nless x_{1}, x_{2}, \ldots, x_{n} \ngtr, n \geq 1$, obtemos uma cadeia infinita de $k$-subálgebras com divisão de $D$,

$$
D_{1} \varsubsetneqq D_{2} \varsubsetneqq \cdots \varsubsetneqq D_{2} \varsubsetneqq \cdots
$$

Se $R=D \otimes_{k} D^{o p}$ então, pelo Lema 6.1.5, $R$ contém uma cadeia infinita de ideais à esquerda e, portanto, $R$ não é noetheriano à esquerda. 


\subsection{O corpo de Weyl}

Seja $k$ um corpo comutativo de característica zero. Makar-Limanov provou em [29] que o corpo de Weyl $k(x)\left(y ; \frac{d}{d x}\right)$ contém uma álgebra livre não-comutativa sobre $k$ e, conseqüentemente, contém um corpo de frações dessa álgebra livre. No entanto, usando o Lema 6.1.8, Lichtman observou que esse corpo de frações não é um corpo livre, como veremos a seguir.

Seja $A_{1}(k)$ a primeira álgebra de Weyl sobre $k$ e seja $W_{1}$ seu corpo clássico de frações à esquerda. Considere $W_{1} \otimes_{k} W_{1}^{o p}$. Podemos construir esta última álgebra em dois passos: primeiro, tome $S:=A_{1}(k) \otimes_{k} W_{1}^{o p}$. Mostremos que $S$ é uma álgebra de Weyl sobre o corpo $W_{1}^{o p}$, isto é, que

$$
S \cong A_{1}\left(W_{1}^{o p}\right)=W_{1}^{o p}\langle z, t: z t-t z=1\rangle \cong W_{1}^{o p}[z]\left[t ; 1, \frac{d}{d z}\right]
$$

Seja $f: k\langle x, y\rangle \longrightarrow A_{1}\left(W_{1}^{o p}\right)$ o único homomorfismo de $k$-álgebras tal que $f(x)=z$ e $f(y)=t$. Então $f(x y-y x-1)=z t-t z-1=0$, o que implica que $\langle x y-y x-1\rangle \subset \operatorname{Ker}(f)$. Logo existe um único homomorfismo de $k$-álgebras $\bar{f}: A_{1}(k) \longrightarrow A_{1}\left(W_{1}^{o p}\right)$ tal que $\bar{f}(x)=z$ e $\bar{f}(y)=t$, onde, com abuso de notação, escrevemos $x$ e $y$ também para suas imagens em $A_{1}(k)$. Seja $g: W_{1}^{o p} \longrightarrow A_{1}\left(W_{1}^{o p}\right)$ a inclusão canônica, que é um homomorfismo de $k$-álgebras. Se $\alpha \in W_{1}^{o p}$ e $\beta \in k \subset Z\left(W_{1}^{o p}\right)$ então

$$
\begin{aligned}
& \bar{f}(x) g(\alpha)=z \alpha=\alpha z=g(\alpha) \bar{f}(x) \\
& \bar{f}(y) g(\alpha)=t \alpha=\alpha t=g(\alpha) \bar{f}(y) \\
& \bar{f}(\beta) g(\alpha)=\beta \alpha=\alpha \beta=g(\alpha) \bar{f}(\beta) .
\end{aligned}
$$

Logo, $\bar{f}(a) g(b)=g(b) \bar{f}(a)$, para todo $a \in A_{1}(k)$, para todo $b \in W_{1}^{o p}$. Portanto existe um único homomorfismo de $k$-álgebras $\varphi: A_{1}(k) \otimes_{k} W_{1}^{o p} \longrightarrow A_{1}\left(W_{1}^{o p}\right)$ tal que $\varphi(a \otimes b)=\bar{f}(a) g(b)$. Como $\varphi(1 \otimes \alpha)=\bar{f}(1) g(\alpha)=g(\alpha)$, para todo $\alpha \in W_{1}^{o p}$, então $\varphi$ é um homomorfismo de $W_{1}^{o p}$-anéis. Agora, como $(x \otimes 1)(1 \otimes \alpha)=(x \otimes \alpha)=(1 \otimes \alpha)(x \otimes 1)$ e $(y \otimes 1)(1 \otimes \alpha)=(y \otimes \alpha)=(1 \otimes \alpha)(y \otimes 1)$, para todo $\alpha \in W_{1}^{o p}$, então existe um único homomorfismo de $W_{1}^{o p}$-anéis $\psi: W_{1}^{o p}\langle z, t\rangle \longrightarrow A_{1}(k) \otimes_{k} W_{1}^{o p}$ tal que $\psi(z)=x \otimes 1$ e $\psi(t)=y \otimes 1$. (Em particular, $\psi$ é um homomorfismo de $k$-álgebras.) Como $\psi(z t-t z-1)=(x \otimes 1)(y \otimes 1)-(y \otimes 1)(x \otimes 1)-1 \otimes 1=x y \otimes 1-y x \otimes 1-1 \otimes 1=(x y-y x-1) \otimes 1=0 \otimes 1=0$ então $\langle z t-t z-1\rangle \subset \operatorname{Ker}(\psi)$. Logo existe um único homomorfismo de $W_{1}^{o p}$-anéis $\bar{\psi}: A_{1}\left(W_{1}^{o p}\right) \longrightarrow$ 
$A_{1}(k) \otimes_{k} W_{1}^{o p}$ tal que $\bar{\psi}(z)=x \otimes 1$ e $\bar{\psi}(t)=y \otimes 1$. Seja $\alpha \in W_{1}^{o p}$. Temos:

$$
\begin{aligned}
& \varphi \bar{\psi}(z)=\varphi(x \otimes 1)=\bar{f}(x) g(1)=z \cdot 1=z, \\
& \varphi \bar{\psi}(t)=\varphi(y \otimes 1)=\bar{f}(y) g(1)=t \cdot 1=t, \\
& \varphi \bar{\psi}(\alpha)=\varphi(1 \otimes \alpha)=\bar{f}(1) g(\alpha)=\alpha,
\end{aligned}
$$

o que implica que $\varphi \bar{\psi}=1_{A_{1}\left(W_{1}^{o p}\right)}$. E, também,

$$
\begin{aligned}
& \bar{\psi} \varphi(x \otimes \alpha)=\bar{\psi}(\bar{f}(x) g(\alpha))=\bar{\psi}(z \alpha)=(x \otimes 1)(1 \otimes \alpha)=x \otimes \alpha, \\
& \bar{\psi} \varphi(y \otimes \alpha)=\bar{\psi}(\bar{f}(y) g(\alpha))=\bar{\psi}(t \alpha)=(y \otimes 1)(1 \otimes \alpha)=y \otimes \alpha,
\end{aligned}
$$

de onde segue que $\bar{\psi} \varphi=1_{S}$. Portanto $\varphi$ é um isomorfismo de $W_{1}^{o p}$-anéis com inversa $\bar{\psi}$. Logo $S \cong A_{1}\left(W_{1}^{o p}\right)$ como $W_{1}^{o p}$-anéis, em particular, como $k$-álgebras.

Já sabemos que $A_{1}\left(W_{1}^{o p}\right)$ é um domínio noetheriano à direita e à esquerda (pela Proposição 1.6.9). Logo $S$ também é. Considere o conjunto $T:=A_{1}(k)^{*} \otimes_{k} 1 \subset S$. Obviamente, $T$ é um conjunto multiplicativo, isto é, $1 \in T, 0 \notin T$ e, se $a, b \in T$, então $a b \in T$. Mostremos que $T$ é um conjunto de Ore à esquerda em $S$, ou seja, que $T\left(\sum a_{i} \otimes \alpha_{i}\right) \cap S(a \otimes 1) \neq \varnothing$, para todo $\sum a_{i} \otimes \alpha_{i} \in S$, para todo $a \in A_{1}(k)^{*}$. Se $\sum a_{i} \otimes \alpha_{i}=0$ então $0 \in T \cdot 0 \cap S(a \otimes 1)$. Suponhamos então que $\sum a_{i} \otimes \alpha_{i} \neq 0$. Sem perda de generalidade podemos supor que $a_{i} \neq 0$ e $\alpha_{i} \neq 0$ para todo $i$. Como $A_{1}(k)$ é um domínio de Ore à esquerda então $A_{1}(k) a_{i} \cap A_{1}(k) a \neq 0$, para todo $i$. Logo existem $c_{i}, b_{i} \in A_{1}(k)^{*}$ tais que $c_{i} a_{i}=b_{i} a$. Em $W_{1}$ temos $a_{i}=c_{i}^{-1}\left(b_{i} a\right)=\left(c_{i}^{-1} b_{i}\right) a$ o que implica $a_{i} a^{-1}=c_{i}^{-1} b_{i}$. Novamente, como $A_{1}(k)$ é Ore à esquerda, podemos colocar os elementos $c_{i}^{-1} b_{i}$ num denominador comum, ou seja, existem $b_{i}^{\prime}, c \in A_{1}(k)^{*}$ tais que $c_{i}^{-1} b_{i}=c^{-1} b_{i}^{\prime}$, para todo $i$. Assim, $a_{i} a^{-1}=c^{-1} b_{i}^{\prime}$ o que implica que $c a_{i}=b_{i}^{\prime} a$, para todo $i$. Logo

$$
0=\sum_{i}\left(c a_{i}-b_{i}^{\prime} a\right) \otimes \alpha_{i}=\sum_{i} c a_{i} \otimes \alpha_{i}-\sum_{i} b_{i}^{\prime} a \otimes \alpha_{i}=\sum_{i}(c \otimes 1)\left(a_{i} \otimes \alpha_{i}\right)-\sum_{i}\left(b_{i}^{\prime} \otimes \alpha_{i}\right)(a \otimes 1) .
$$

Logo, $r:=(c \otimes 1) \sum_{i}\left(a_{i} \otimes \alpha_{i}\right)=\left(\sum_{i} b_{i}^{\prime} \otimes \alpha_{i}\right)(a \otimes 1)$, com $c \otimes 1 \in T$ e $\sum_{i} b_{i}^{\prime} \otimes \alpha_{i} \in S$. Portanto $r \in T\left(\sum a_{i} \otimes \alpha_{i}\right) \cap S(a \otimes 1)$. Consideremos a localização de $S$ em $T$,

$$
T^{-1} S=\left(A_{1}(k)^{*} \otimes_{k} 1\right)^{-1}\left(A_{1}(k) \otimes_{k} W_{1}^{o p}\right)=\left(A_{1}(k)^{*-1} \otimes_{k} 1\right)\left(A_{1}(k) \otimes_{k} W_{1}^{o p}\right) \subset W_{1} \otimes_{k} W_{1}^{o p} .
$$

Se $\alpha, \beta \in W_{1}, \alpha=b^{-1} a$, então $\alpha \otimes \beta=b^{-1} a \otimes \beta=\left(b^{-1} \otimes 1\right)(a \otimes \beta) \in T^{-1} S$. Logo, $T^{-1} S=W_{1} \otimes_{k} W_{1}^{o p}$. 
Como $S$ é noetheriano à direita e à esquerda então $T^{-1} S$ também é, pela Proposição 4.10.32 de [19]. Provamos então que $W_{1} \otimes_{k} W_{1}^{o p}$ é um anel noetheriano à esquerda (e à direita). Pelo Lema 6.1.8, $W_{1}$ não contém um corpo livre (não-comutativo) sobre $k$, ou seja, não contém um corpo livre da forma $k_{C} \nless X \ngtr,|X| \geq 2$, para todo subcorpo $C$ de $k$. Em particular, $W_{1}$ não contém o corpo livre $k \nless X \ngtr$, $|X| \geq 2$ e, portanto, não contém nenhum corpo livre sobre qualquer subcorpo central, pelo Lema 2.2.6. Obtivemos então o seguinte

Teorema 6.2.1. O corpo de Weyl sobre um corpo comutativo $k, k(x)\left(y ; \frac{d}{d x}\right)$, não contém nenhum corpo livre (sobre qualquer subcorpo).

\subsection{O corpo de frações de $K G$}

Seja $G$ um grupo nilpotente, finitamente gerado, livre de torção, não-abeliano, e seja $k$ um corpo comutativo. O mesmo método da seção anterior pode ser usado para mostrar que o corpo de frações $Q(k G)$ da álgebra de grupo $k G$ não contém um corpo livre sobre $k$. Denotemos por $F$ o corpo clássico de frações à esquerda de $k G$. O anel de grupo $F G$ é também uma $k$-álgebra e portanto podemos considerar a $k$-álgebra $R=k G \otimes_{k} F^{o p}$. Mostremos que $R \cong F^{o p} G$. Sejam $\phi: k G \longrightarrow F^{o p} G$ e $\psi: F^{o p} \longrightarrow F^{o p} G$ as inclusões naturais que, obviamente, são homomorfismos de $k$-álgebras. Mais ainda, $\psi$ é um homomorfismo de $F^{o p}$-anéis. Se $f=\sum_{g \in G} \alpha_{g} g \in k G$ e $\beta \in F^{o p}$ então

$$
\begin{aligned}
\phi(f) \psi(\beta) & =f \beta=\left(\sum \alpha_{g} g\right) \beta=\left(\sum g \alpha_{g}\right) \beta=\sum g\left(\alpha_{g} \beta\right)=\sum g\left(\beta \alpha_{g}\right)=\sum(g \beta) \alpha_{g} \\
& =\sum(\beta g) \alpha_{g}=\beta \sum g \alpha_{g}=\beta \sum \alpha_{g} g=\psi(\beta) \phi(f) .
\end{aligned}
$$

Logo existe um único homomorfismo de $k$-álgebras $\theta: k G \otimes_{k} F^{o p} \longrightarrow F^{o p} G$ tal que $\theta(f \otimes \beta)=$ $\phi(f) \psi(\beta)=f \beta=\beta f$, para todo $f \in k G$, para todo $\beta \in F^{o p}$. Como $\theta(1 \otimes \beta)=\beta=\psi(\beta)$ então $\theta$ é um homomorfismo de $F^{o p}$-anéis. Seja $\xi: F^{o p} G \longrightarrow k G \otimes_{k} F^{o p}$ o único homomorfismo de $F^{o p}$-espaços à direita tal que $\xi(g)=g \otimes 1$, para todo $g \in G$. Se $f=\sum_{g \in G} \alpha_{g} g \in k G, h=\sum_{g \in G} \beta_{g} g \in F^{o p} G$ e $\beta \in F^{o p}$ então

$$
\begin{gathered}
\xi \theta(f \otimes \beta)=\xi(f \beta)=\xi\left(\sum g\left(\alpha_{g} \beta\right)\right)=\sum(g \otimes 1) \alpha_{g} \beta=\sum\left(g \otimes \alpha_{g} \beta\right) \\
=\sum(g \alpha g \otimes \beta)=\left(\sum g \alpha_{g}\right) \otimes \beta=f \otimes \beta, \\
\theta \xi(h)=\theta \xi\left(\sum g \beta_{g}\right)=\theta\left(\sum(g \otimes 1) \beta_{g}\right)=\theta\left(\sum g \otimes \beta_{g}\right)=\sum g \beta_{g}=h,
\end{gathered}
$$


e, portanto $\theta$ é um isomorfismo com inversa $\xi$. Logo $F^{o p} G \cong R$ como $F^{o p}$-anéis, em particular, como $k$-álgebras. Como $F^{o p}$ é um domínio noetheriano à direita e à esquerda, então $R$ também é.

Procedendo de maneira análoga ao que foi feito na seção anterior, é possível mostrar que $F \otimes_{k} F^{o p}$ é um anel noetheriano à esquerda (e à direita). Pelo Lema 6.1.8, F não contém um corpo livre (nãocomutativo) sobre $k$. Obtivemos então o seguinte

Teorema 6.3.1. O corpo de frações $Q(k G)$ da álgebra de grupo $k G$ de um grupo nilpotente, finitamente gerado, livre de torção, não-abeliano $G$, sobre um corpo comutativo $k$, não contém nenhum corpo livre (sobre qualquer subcorpo).

Makar-Limanov provou em [30] que $Q(k G)$ contém uma álgebra livre não-comutativa sobre $k$ e, conseqüentemente, contém um corpo de frações dessa álgebra livre. No entanto, esse corpo de frações não é o corpo livre, pelo que acabamos de mostrar. 
76 CAPÍTULO 6. ALGUNS ANÉIS COM DIVISÃO QUE NÃO CONTÊM CORPOS LIVRES 


\section{Capítulo 7}

\section{Considerações Finais}

Tratamos neste trabalho da existência de imersões de corpos livres em completamentos, com respeito a uma métrica induzida por uma valorização, de anéis com divisão de dimensão infinita sobre seus centros. Grande parte dos anéis considerados aqui são corpos de frações de domínios noetherianos com uma filtração natural.

O principal resultado provado garante a existência de corpos livres em completamentos de anéis com divisão com uma valorização discreta, cujo centro é infinito e a dimensão sobre o centro é infinita (Teorema 2.2.4). Dentre esses completamentos estão, por exemplo:

- corpos de séries de Laurent sobre um anel com divisão (Seção 2.2);

- corpos de séries de Malcev-Neumann de grupos nilpotentes, finitamente gerados, livres de torção (Capítulo 3);

- alguns corpos de séries de Laurent skew como, por exemplo, um completamento do corpo de Weyl em característica zero (Capítulo 4);

- o completamento de um corpo de frações da envolvente universal de uma álgebra de Lie em característica zero com relação a valorização canônica (Capítulo 5).

Como é sabido, a existência de uma álgebra livre em um anel com divisão $D$ não garante a existência de um corpo livre em $D$, mesmo se $D$ tiver um completamento $\hat{D}$ que contenha um corpo livre. Os corpos de frações da primeira álgebra de Weyl em característica zero e da álgebra de grupo de um grupo nilpotente, finitamente gerado, livre de torção, não-abeliano sobre um corpo comutativo 
são exemplos deste fato, como foi visto no Capítulo 6. Cabe aqui nos questionar se esse é um fato geral, ou seja, será que todo anel com divisão que contém um álgebra livre tem uma valorização cujo completamento associado contém um corpo livre?

Nossos resultados garantem a existência de um corpo livre em determinados anéis com divisão, porém não fornecem uma construção explícita dos geradores desse corpo livre. A procura por resultados que nos forneçam tais geradores, principalmente em anéis com divisão que já sabemos que contêm um corpo livre, é algo de nosso interesse.

Outras questões que também surgiram no decorrer do trabalho e que nos indicam algumas direções de pesquisa futura são:

- Será que os métodos utilizados no Capítulo 3 se aplicam para grupos poli- $\mathbb{Z}$ quaisquer ou, mais geralmente, para grupos policíclicos? E para grupos residualmente-\{nilpotente, livre de torção\}?

- Como indicado na Seção 3.2, uma resposta à segunda questão acima pode eventualmente ser obtida como conseqüência do Teorema 2.3.1. Quais seriam outras possíveis aplicações desse teorema?

- Neste trabalho fizemos uso de valorizações discretas em anéis com divisão para construção de anéis com divisão que contêm corpos livres. No caso de valorizações em anéis com divisão que não são necessariamente discretas ou mesmo abelianas, algo semelhante poderia ser feito?

- Sejam $K \subset D_{1} \subset D_{2}$ onde $D_{1}$ e $D_{2}$ são anéis com divisão tais que $\operatorname{dim} D_{2 D_{1}}<\infty$ ou $\operatorname{dim} D_{D_{1}} D_{2}<$ $\infty$ e $K$ é um subcorpo central de $D_{2}$. Se $D_{2}$ contém um corpo livre $K \nless X \ngtr$ então $D_{1}$ também contém? Esta pergunta nos foi feita por Makar-Limanov e a resposta também não é conhecida se trocamos $K \nless X \ngtr$ por $K\langle X\rangle$. Um problema relacionado a este foi considerado por Reichstein em [38]. 


\section{Referências Bibliográficas}

[1] Bourbaki, N., Commutative Algebra, Chapters I-VII, Addison-Wesley, Reading, Mass., 1972.

[2] Chiba, K., Free subgroups and free subsemigroups of division rings, J. Algebra 184 (1996), $570-574$.

[3] Chiba, K., Free fields in complete skew fields and their valuations, J. Algebra 263 (2003), 75-87.

[4] Cohn, P. M., On the embedding of rings in skew fields, Proc. London. Math. Soc. 11 (1961), $511-530$.

[5] Cohn, P. M., Skew Field Constructions, Cambridge University Press, Cambridge, 1977.

[6] Cohn, P. M., Free Rings and Their Relations, 2nd. Ed., Academic Press, London, 1985.

[7] Cohn, P. M., Algebra, 2nd. Ed., Vol. III, John Wiley and Sons, Chichester, 1991.

[8] Cohn, P. M., Skew Fields. Theory of General Division Rings, Cambridge University Press, Cambridge, 1995.

[9] Dauns, J., A Concrete Approach to Division Rings, Heldermann Verlag, Berlin, 1982.

[10] Ferreira, V. O., Gonçalves, J. Z. and Mandel, A., Free symmetric and unitary pairs in division rings with involution, Internat. J. Algebra Comput. 15 (2005), 15-36.

[11] Gonçalves, J. Z., Free subgroups of units in group rings, Canad. Math. Bull. 27 (1984), 309-312.

[12] Gonçalves, J. Z., Free groups in subnormal subgroups and the residual nilpotence of the group of units of group rings, Canad. Math. Bull. 27 (1984), 365-370.

[13] Gonçalves, J. Z. and Mandel, A., Are there free groups in division rings?, Israel J. Math. 53 (1986), 69-80.

[14] Jacobson, N., Lectures in Abstract Algebra III. Theory of Fields and Galois Theory, SpringerVerlag, New York, 1975. 
[15] Jacobson, N., Lie Algebras, Dover Publications, Inc., New York, 1979.

[16] Jennings, S. A., The group ring of a class of infinite nilpotent groups, Can. J. Math. 7 (1955), 169-187.

[17] Kassel, C., Quantum Groups, Springer-Verlag, New York, 1995.

[18] Lam, T. Y., A First Course in Noncommutative Rings, Springer-Verlag, New York, 1991.

[19] Lam, T. Y., Lectures on Modules and Rings, Springer-Verlag, New York, 1999.

[20] Lewin, J., Fields of fractions for group algebras of free groups, Trans. Amer. Math. Soc. 192 (1974), 339-346.

[21] Lichtman, A. I., On subgroups of the multiplicative group of skew fields, Proc. Amer. Math. Soc 63 (1977), 15-16.

[22] Lichtman, A. I., Free subgroups of normal subgroups of the multiplicative group of skew fields, Proc. Amer. Math. Soc. 71 (1978), 174-178.

[23] Lichtman, A. I., On normal subgroups of the multiplicative group of skew fields generated by a polycyclic-by-finite group, J. Algebra 78 (1982), 548-577.

[24] Lichtman, A. I., Free subgroups in linear groups over some skew fields, J. Algebra 105 (1987), $1-28$.

[25] Lichtman, A. I., Valuation methods in division rings, J. Algebra 177 (1995), 870-898.

[26] Lichtman, A. I., Free subalgebras in division rings generated by universal enveloping algebras, Algebra Colloq. 6 (1999), 145-153.

[27] Lorenz, M., Group rings and division rings, Methods in Ring Theory (Antwerp, 1983), 265-280, NATO Adv. Sci. Inst. Ser. C Math. Phys. Sci., 129, Reidel, Dordrecht, 1984.

[28] Lorenz, M., On free subalgebras of certain division algebras, Proc. Amer. Math. Soc. 98 (1986), 401-405.

[29] Makar-Limanov, L., The skew field of fractions of the Weyl algebra contains a free noncommutative subalgebra, Comm. Alg. 11 (1983), 2003-2006.

[30] Makar-Limanov, L., On group rings of nilpotent groups, Israel J. Math. 48 (1984), 244-248.

[31] Makar-Limanov, L., On free subobjects of skew fields, Methods in Ring Theory (Antwerp, 1983), 281-285, NATO Adv. Sci. Inst. Ser. C Math. Phys. Sci., 129, Reidel, Dordrecht, 1984.

[32] Makar-Limanov, L., On subnormal subgroups of skew fields, J. Algebra 114 (1988), 261-267. 
[33] Makar-Limanov, L. and Malcolmson, P., Free subalgebras of enveloping fields, Proc. Amer. Math. Soc. 111 (1991), 315-322.

[34] Passmann, D. S., The Algebraic Structure of Group Rings, Wiley-Interscience, New York, 1977.

[35] Passmann, D. S., Infinite Crossed Products, Academic Press, Boston, Mass., 1989.

[36] Pierce, R. S., Associative Algebras, Springer-Verlag, New York, 1982.

[37] Polcino Milies, C. and Sehgal, S. K., An Introduction to Group Rings, Kluwer, Dordrecht, 2002.

[38] Reichstein Z., On a question of Makar-Limanov, Proc. Amer. Math. Soc. 124 (1996), 17-19.

[39] Reichstein, Z. and Vonessen, N., Free subgroups of division algebras, Comm. Algebra 23 (1995), 2181-2185.

[40] Rowen, L. H., Ring Theory, Vol. I, Academic Press, Boston, Mass., 1988.

[41] Schilling, O. F. G., The Theory of Valuations, American Mathematical Society, New York, 1950.

[42] Shirvani, M. and Gonçalves, J. Z., Free group algebras in the field of fractions of differential polynomial rings and enveloping algebras, J. Algebra 204 (1998), 372-385.

[43] Shirvani, M. and Gonçalves, J. Z., Large free algebras in the ring of fractions of skew polynomial rings, J. London Math. Soc. (2) 60 (1999), 481-489.

[44] Snider, R. L., The division ring of fractions of a group ring, Paul Dubreil and Marie-Paule Malliarin Algebra Seminar, 35th year (Paris, 1982), 325-339, Lectures Notes in Math. 1029, Springer, Berlin, 1983. 


\section{Índice Remissivo}

$D$-anel livre sobre $K$ em $X, 11$

$R$-anel, 6

$R$-corpo, 6

épico, 6

universal, 7

$\mathcal{F}$-série, 19, 43

álgebra

de Lie, 29

de matrizes quânticas, 55

de Weyl, 28, 72

livre, 10,57

anel

$\Sigma$-inversor universal, 8

clássico de frações à direita, 14

com divisão, 3

de frações à direita, 13

de grupo, 22

skew, 22

de operadores diferenciais, 23

de Ore, 14

de Ore à direita, 14, 15

de polinômios, 15, 16, 23

skew, 23

diferenciáveis, 23

de valorização, 17

filtrado, 61

graduado, 61

completamento, 17

conjunto

bem ordenado, 21

de Ore à direita, 14

denominador à direita, 14

enumerável, 3

multiplicativo, 13

reversível à direita, 14

corpo, 3

das funções racionais, 15

skew, 24

das matrizes quânticas, 49

das séries de Laurent, 17, 32, 34

skew, 25, 26, 49

das séries de Malcev-Neumann, 22, 37

skew, 22, 46

de frações, 6, 13

de Lie, 30, 60

de resíduos, 17

de Weyl, 28, 54, 65, 72

livre, 12

primo, 3

universal, 7

derivação, 23

domínio, 3

de ideais principais à direita, 15, 23

de ideais principais à esquerda, 15, 23

de Ore à direita, 15, 28

de Sylvester, 10, 12

envolvente universal, 30, 60

especialização, 6, 35 
extensão de Ore, 23

filtração, 61

negativa, 61

positiva, 61

forma normal, 20

grupo

nilpotente, 18, 37, 65, 74

ordenado, 15

homomorfismo

$\Sigma$-inversor, 7

universal, 7

honesto, 10

local, 6

plenamente inversor, 10

localização à direita, 13

localização universal, 8

módulo fielmente plano, 65

matriz plena, 9

à direita, 9

à esquerda, 9

ordem interna (de um automorfismo), 52

Poincaré-Birkhoff-Witt, 30, 62

posto interno, 9

propriedade universal

de álgebras livres, 10

de anéis livres, 11

de anéis de polinômios skew, 23

regra de Leibniz, 24

série central, 18

inferior, 19

superior, 19

suporte, 22

valorização, 16 t-ádica, 16, 59

abeliana, 17

discreta, 17

própria, 16 